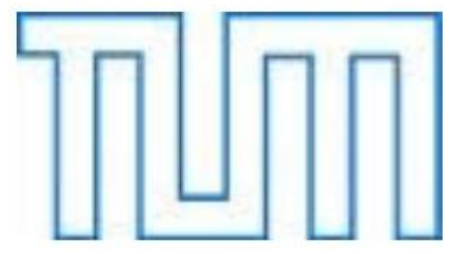

Dissertation der Fakultät für Medizin der

Technischen Universität München

Lehrstuhl für Herz- und Gefäßchirurgie

\title{
TOTALE LUNGENVENENFEHLMÜNDUNG
}

\author{
Caroline Maria Neuray
}

Vollständiger Abdruck der von der Fakultät für Medizin der Technischen Universität

München zur Erlangung des akademischen Grades eines Doktors der Medizin

genehmigten Dissertation

Vorsitzender:

Prof. Dr. Jürgen Schlegel

Prüfer der Dissertation:

1. apl. Prof. Dr. Jürgen Hörer

2. Prof. Dr. Rüdiger Lange

Die Dissertation wurde am 25.10.2016 bei der Technischen Universität München eingereicht und durch die Fakultät für Medizin am 09.01.2018 angenommen. 


\section{Inhaltsverzeichnis}

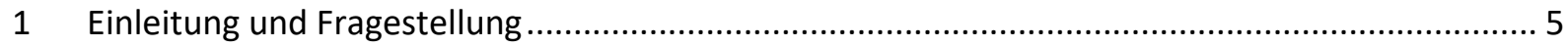

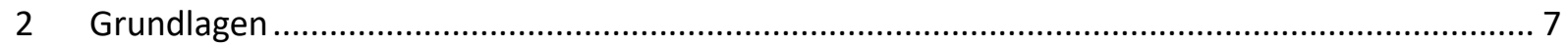

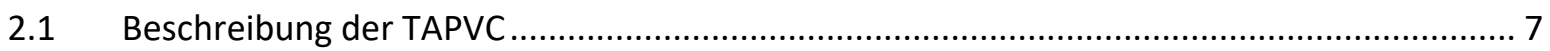

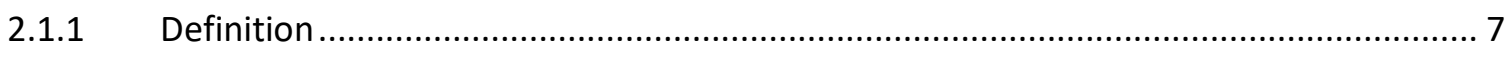

2.1.2 Anatomie und Pathophysiologie ............................................................................ 7

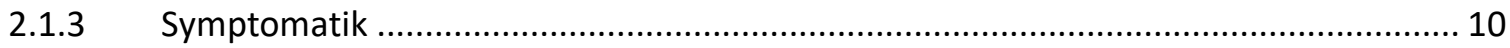

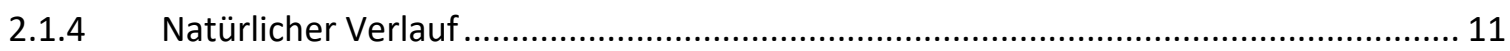

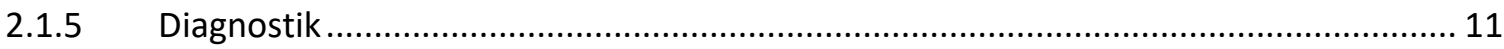

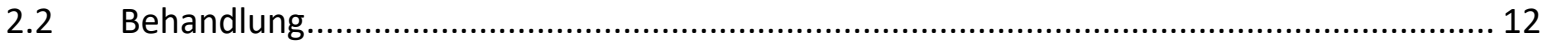

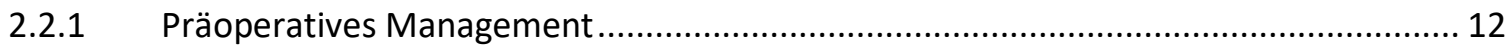

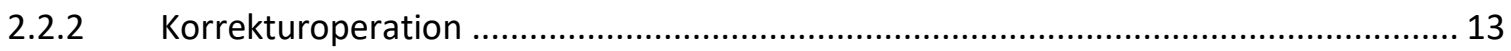

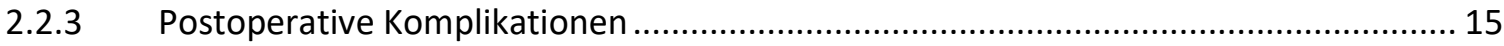

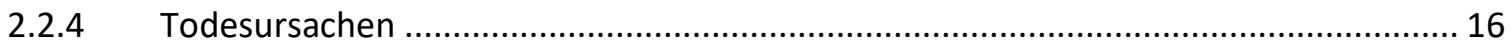

2.3 Historische Wandlung der Operationstechnik ................................................................ 16

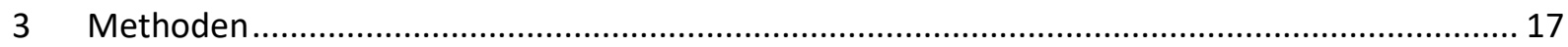

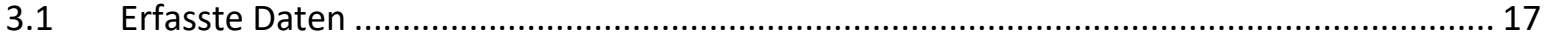

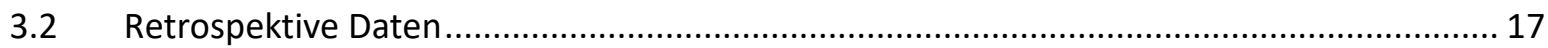

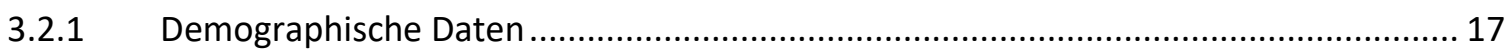

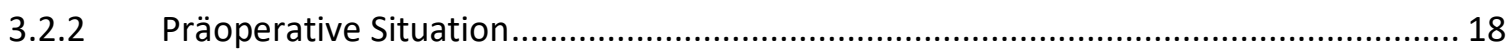

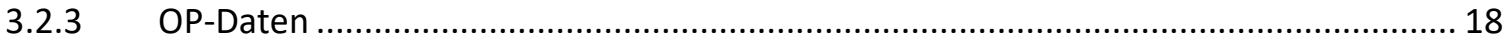

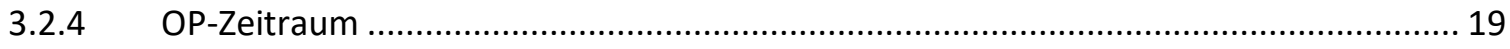

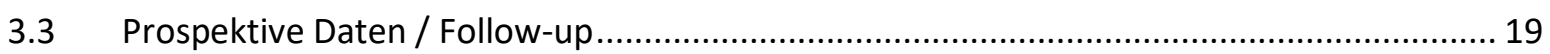

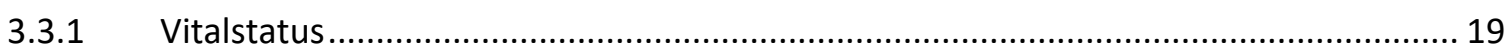

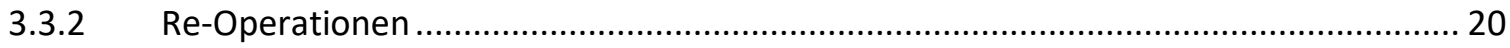

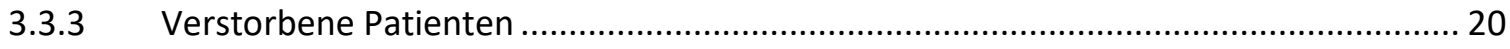

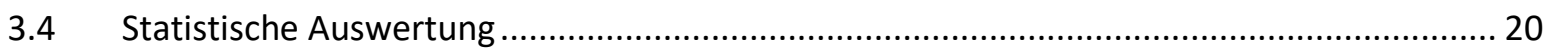

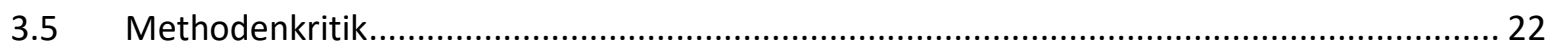

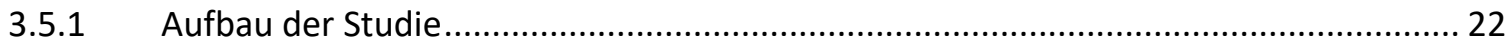

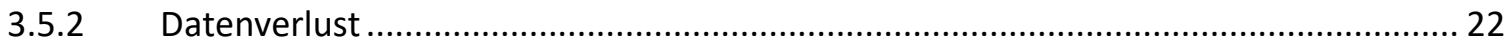

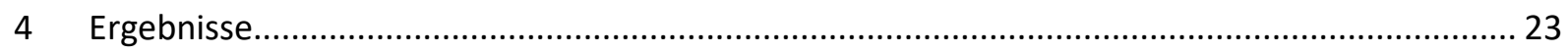

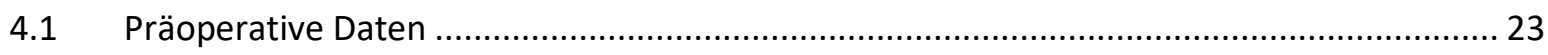




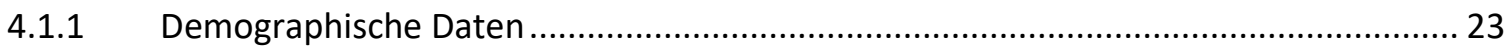

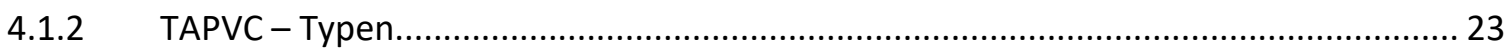

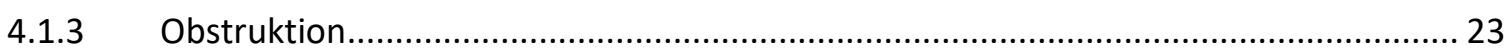

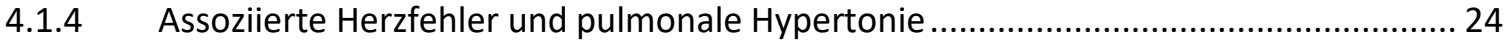

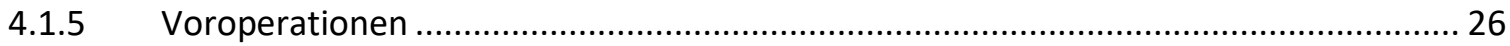

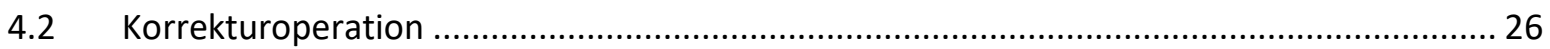

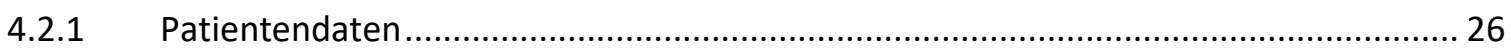

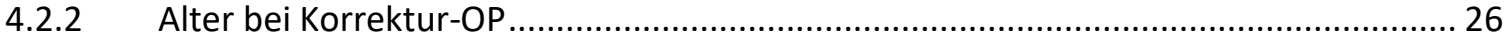

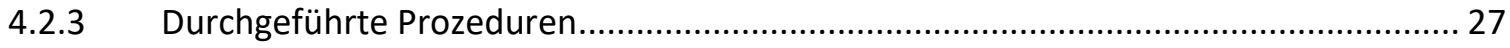

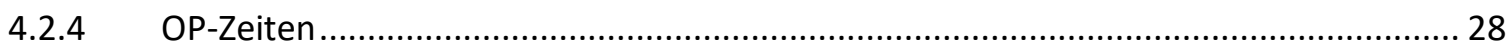

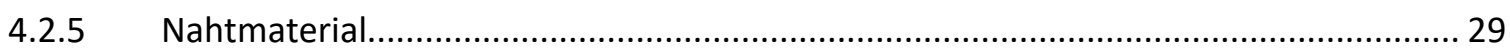

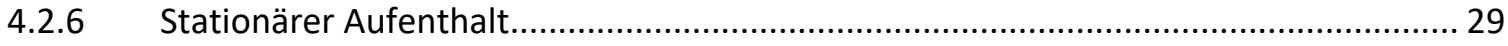

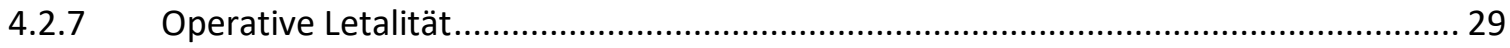

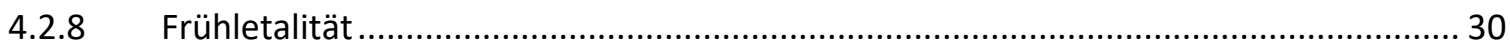

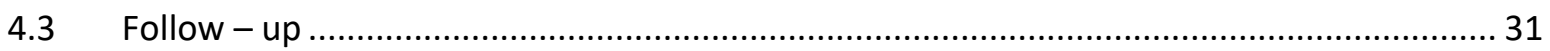

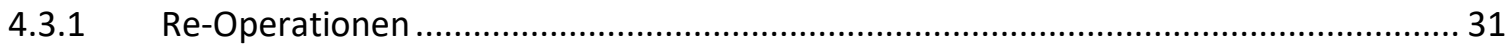

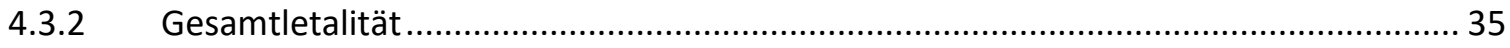

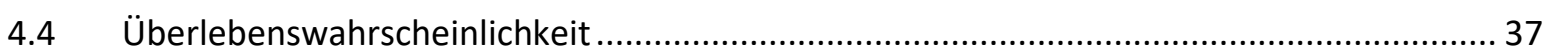

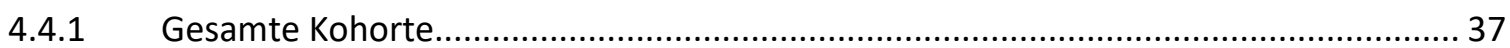

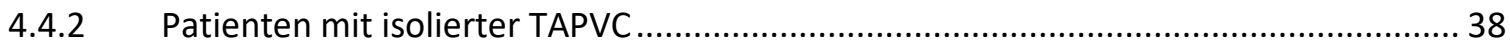

4.4.3 Patienten mit präoperativ bestehender pulmonaler Hypertonie.............................. 38

4.4.4 Patienten mit zusätzlichen Anomalien des Herzens (komplexe Herzfehler) ................ 38

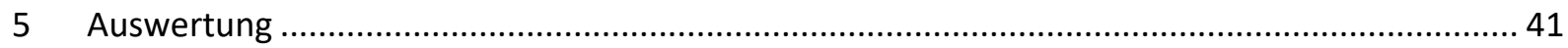

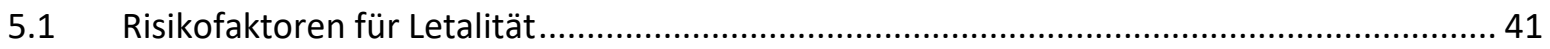

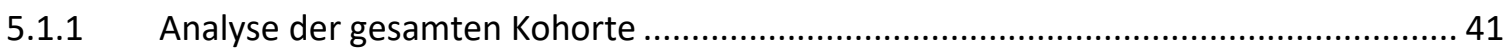

5.1.2 Analyse der Patienten mit isolierter TAPVC ....................................................... 42

5.2 Risikofaktoren für Re-Operationen wegen postoperativer PVO .................................. 44

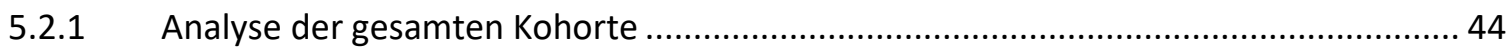

5.2.2 Analyse der Patienten mit isolierter TAPVC .................................................. 45

5.3 Risikofaktoren für kardiale Re-Operationen wegen andere Indikation ........................... 46

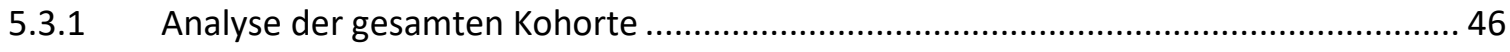

5.3.2 Analyse der Patienten mit isolierter TAPVC .................................................... 47 
6 Diskussion

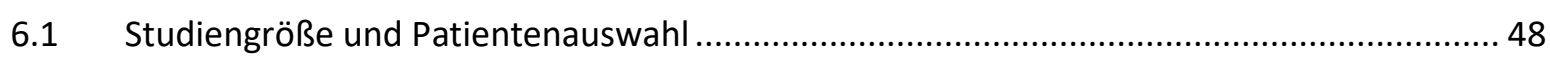

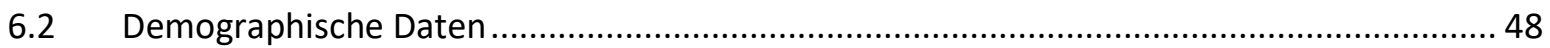

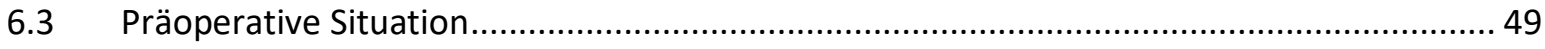

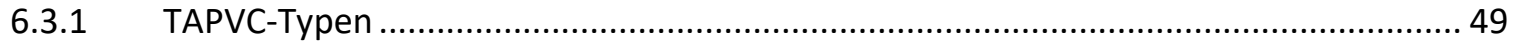

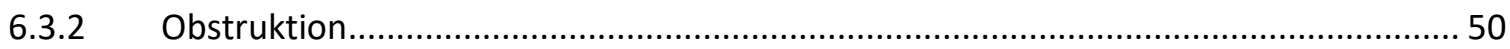

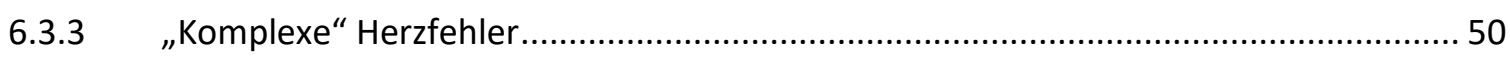

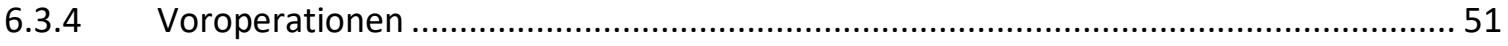

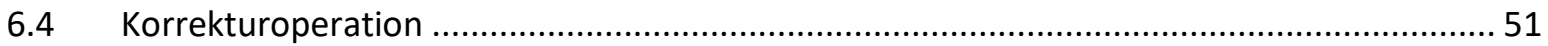

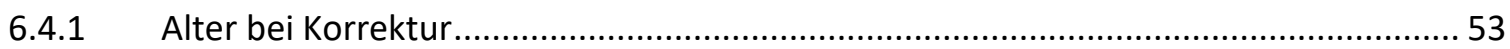

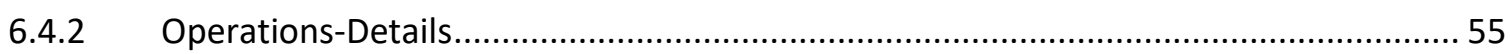

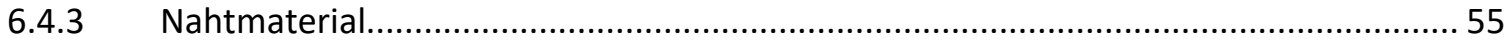

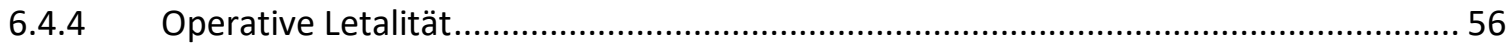

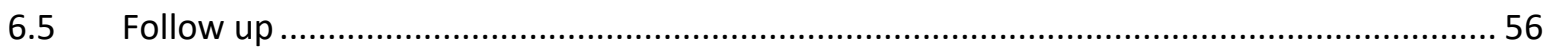

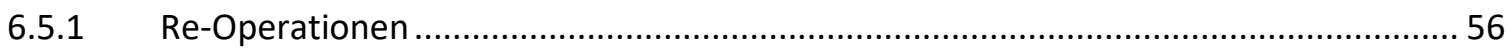

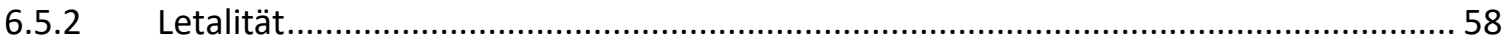

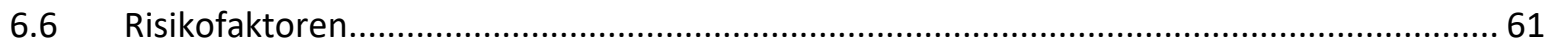

6.6.1 Risikofaktoren für geringere Überlebenswahrscheinlichkeit ..................................... 61

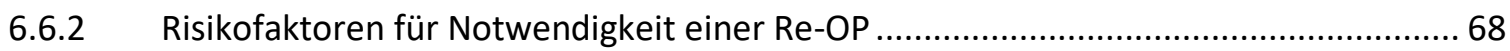

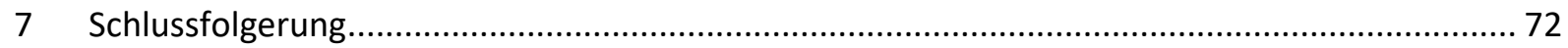

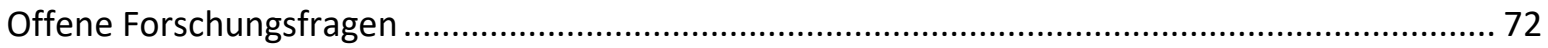

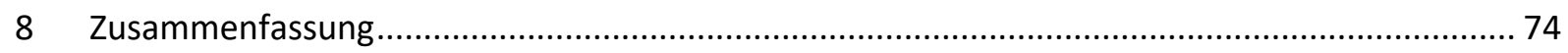

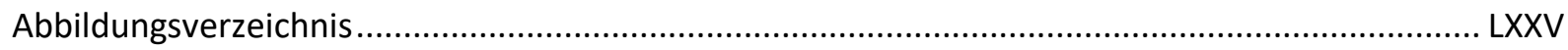

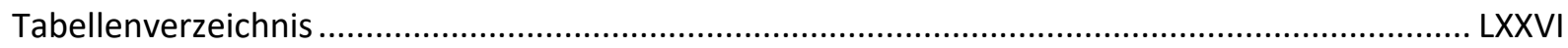

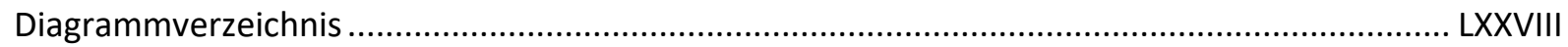

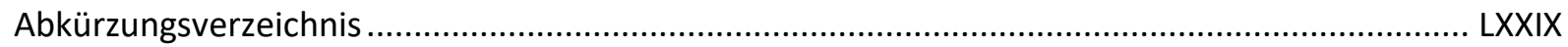

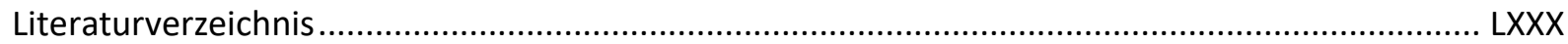




\section{Einleitung und Fragestellung}

Diese Arbeit befasst sich mit einem seltenen, angeborenen Herzfehler, der totalen Lungenvenenfehlmündung (TAPVC). Angeborene Fehlbildungen des Herzens treten in etwa bei $0,5 \%$ bis $0,8 \%$ der Lebendgeborenen auf. Davon macht die totale Lungenvenenfehlbildung nur 1 bis 3\% aller kardialen Anomalien aus (Michielon et al., 2002). Der natürliche Verlauf ist sehr schlecht und ohne chirurgische Korrektur nahezu immer tödlich (80\% bis $96 \%$ im ersten Lebensjahr). (Burroughs \& Edwards, 1960; Keith, Rowe, Vlad, \& O'Hanley, 1954; Samanek, Benesova, Goetzova, \& Hrycejova, 1988).

Seit der Erstbeschreibung 1798 gelang erst 1951 die erste operative Korrektur (J. Wilson, 1798). Seitdem kam es durch kontinuierliche Weiterentwicklung von operativen Techniken und perioperativer Versorgung zu einer über die Jahre deutlich abnehmenden Letalität (Bando et al., 1996; Lamb et al., 1988). Heutzutage liegt die operative Sterblichkeit der Patienten mit totaler Lungenvenenfehlmündung nach Korrektur bei 0 bis 10\%. (Kirshbom et al., 2002; Macartney, Taylor, Graham, De Leval, \& Stark, 1980)

Neben der zunehmenden chirurgischen Erfahrung trugen auch verbesserte Techniken der extrakorporalen Zirkulation und bessere intensivmedizinische Betreuung zur Abnahme der Letalität bei. Viele andere potentielle Risikofaktoren wurden untersucht und in der Literatur diskutiert. Darunter stehen an erster Stelle die anatomischen Gegebenheiten. Hier unterscheidet man je nach Mündung der fehlkonnektierten Lungenvenen drei verschiedene Typen, welche wiederum durch unterschiedliche Pathophysiologie verschiedene präoperative Gegebenheiten mit sich bringen. Hierzu gehört an erster Stelle eine eventuelle Obstruktion der Lungenvenen, welche sich in der klinischen Präsentation von milder Zyanose angefangen, bis hin zum neonatologischen Notfall darstellen kann. Ob und wie die verschiedenen Konnektionstypen Einfluss auf die Überlebenswahrscheinlickeit nehmen, wird kontrovers diskutiert. Gängige Meinung ist es mittlerweile, dass eine Mündung der Lungenvenen in abdominelle Gefäße immer mit einer Obstruktion einhergeht. Einige Autoren zeigten dies auch für suprakardial mündende Lungenvenen (Delius, de Leval, Elliott, \& Stark, 1996; Kirshbom et al., 2002).

Auch das Vorhandensein zusätzlicher kardialer Anomalien beeinflusst die Überlebenswahrscheinlichkeit selbst nach Korrektur deutlich; insbesondere die der funktionell univentrikulären Malformationen haben eine sehr schlechte Prognose. Überlebenswahrscheinlichkeiten für diese Patienten mit TAPVC und funktionell univentrikulären Malformationen sind sehr niedrig und gehören in dieser Kombination zu den Herzfehlern mit der schlechtesten Prognose (Gaynor, Collins, Rychik, Gaughan, \& Spray, 1999). Auch zeigten die meisten Autoren eine Stagnation der Prognose für diese Gruppe an Patienten über die letzten Dekaden (Caldarone et al., 1998b).

Bezüglich der Notwendigkeit von späteren Re-Operationen werden insbesondere Unterschiede im chirurgischen Vorgehen als potentielle Einflussgrößen genannt. Als häufigster Grund für ReOperationen wird eine (post-)operativ entstehende Stenosierung der hergestellten Anastomose zwischen Lungenvenen und linkem Vorhof aufgezeigt. Als potentielle Einflussfaktoren diskutiert werden unter Anderem präoperativ bestehende Obstruktion, TAPVC-Typ, Alter bei Korrektur, Jahr der Korrektur und Operationstechniken.

Aufgrund der Seltenheit dieses Herzfehlers und der für eine Korrekturoperation notwendigen Expertise ist die Behandlung traditionell speziellen Zentren vorbehalten. Die hier vorliegende Studie be- 
schreibt und analysiert die Ergebnisse aller Patienten mit totaler Lungenvenenfehlmündung, die am Deutschen Herzzentrum München in den Jahren von 1974 bis 2011 korrigierend operiert wurden. Das Deutsche Herzzentrum München war eine der ersten Kliniken in Deutschland, an der dieser Herzfehler operiert wurde. Die vorliegende Analyse richtete sich nach den Fragen nach potentiellen Faktoren sowohl für peri- und postoperative Letalität als auch für die Notwendigkeit von ReOperationen. Nachdem diese in der Literatur kontrovers diskutiert werden, ist das Ziel der vorliegenden Arbeit die Identifizierung von Risikofaktoren für erhöhte Letalität und für nötige ReOperationen im eigenen Patientengut. Hierzu wurden zahlreiche prä-, peri- und postoperative Daten erhoben und analysiert. Primäre Endpunkte waren dabei das Überleben und die Freiheit von ReOperationen. Diesbezüglich wurden im Laufe der Nachbeobachtung Vitalstatus, stattgehabte ReOperationen und Wohlergehen der Patienten erhoben und in die Analyse miteinbezogen.

Hintergrund dieser Zielsetzung ist die Frage nach Risikofaktoren, insbesondere das Patientenmanagement und das operative Prozedere betreffend, welche ggf. optimiert werden können, um eine Reduktion an Letalitäts- und Re-Operationsraten anstreben zu können.

Im Folgenden werden zunächst allgemein Pathologie, Diagnostik und Operationsverfahren der totalen Lungenvenenfehlmündung beschrieben. Im Hauptteil werden die Ergebnisse der Datenerhebung im Patientengut des Deutschen Herzzentrum München von 1974 bis 2010 dargestellt, die Analyse wiedergegeben und schlussendlich die erhobenen Erkenntnisse diskutiert und mit denen anderer Autoren verglichen. 


\section{Grundlagen}

\subsection{Beschreibung der TAPVC}

\subsubsection{Definition}

Bei der totalen Lungenvenenfehlmündung handelt es sich um einen seltenen angeborenen Herzfehler, der nur 1 bis 3\% (Michielon et al., 2002) der kardialen Anomalien ausmacht und bei 1 von 14700 Lebendgeburten festgestellt wird (Correa-Villasenor, Ferencz, Boughman, \& Neill, 1991). Bei dieser Anomalie münden die Lungenvenen nicht - wie regulär - in den linken Vorhof, sondern in den rechten Vorhof bzw. einen seiner Zuflüsse. Auch wenn ein offenes Foramen ovale bzw. ein ASD per se nicht Teil der Malformation ist, ist das Vorhandensein einer solchen oder anderen intrakardialen Verbindung zwischen linkem und rechtem Herzen für einen überhaupt funktionierenden Blutkreislauf (Lungen- und Systemkreislauf verbunden) und damit das postpartale Überleben essentiell.

Embryologisch kommt diese Fehlbildung durch eine fehlende sekundäre Involution der Lungenvenen zustande. Während der Lungenentwicklung vereint sich der intrapulmonale Lungenvenenplexus mit dem pulmonalvenösen Konfluens, der als primäre Lungenvene das entstehende Mediastinum quert und sich somit dem atrialen Anteil des sich entwickelnden Herzens nähert. Normalerweise fusionieren diese primären Lungenvenen dann mit dem morphologisch linken Atrium. Bleibt diese Fusionierung aus, bleibt der pulmonalvenöse Konfluens als Teil des systemischen Kreislaufs bestehen und vergrößert sich. Je nachdem welche Anastomosen sich im weiteren Verlauf bilden, kommen verschiedene anatomische Varianten der TAPVC zustande (Edwards, 1953; Hancock Friesen et al., 2005; Lock et al., 1984; Neill, 1956; Raisher, Grant, Martin, Strauss, \& Spray, 1992).

Als „monstrous formation of the heart in which the superior caval vein was joined by a trunk formed by two large veins coming out of the lungs" wurde die TAPVC 1798 erstmals von Wilson beschrieben (J. Wilson, 1798). Die erste klinische Beschreibung eines Falles entstand weitaus später (1950). Friedlich konnte hier erstmals die Diagnose angiokardiographisch bestätigen (Friedlich, Bing, \& Blount, 1950).

\subsubsection{Anatomie und Pathophysiologie}

\subsubsection{TAPVC-Typen}

Je nach Mündungsort der fehlkonnektierten Lungenvenen werden verschiedene anatomische Konnektionstypen unterschieden. Man unterscheidet den suprakardialen, den kardialen, den infrakardialen und den gemischten Typ. Dabei werden kardialer und suprakardialer Typ auch als supradiaphragmatische Konnektion und der infrakardiale Typ als infradiaphragmatische Konnektion bezeichnet. Eine große Studie aus dem Children's Hospital in Boston beschreibt anhand von Autopsiebefunden die Häufigkeitsverteilung der unterschiedlichen Konnektionstypen. Dabei waren $45 \%$ suprakardial, 25\% kardial, 25\% infrakardial und 5\% gemischt (Delisle et al., 1976). Unabhängig von der anatomischen Mündungsstelle, ist allen Konnektionsformen gemein, dass sich die Lungenvenen zu- 
nächst hinter dem linken Vorhof zu einem Konfluens vereinen. Von da aus drainiert das Blut dann anomal in die jeweiligen anschließend beschriebenen Gefäße.

Diese anatomische Differenzierung beeinflusst die Pathophysiologie und die Operationstechnik, weshalb sie hier genauer ausgeführt werden soll.

\section{Suprakardiale Konnektion}

Darling et al. beschreibt den suprakardialen Typ bei Mündung des Sammelgefäßes in die Vena anonyma, die Vena cava superior, die Vena azygos oder in die Vena brachiocephalica sinistra. Am häufigsten ist die Mündung in die Vena anonyma, wobei die Lungenvenen in die Vena verticalis ascendens münden, welche dann in die Vena anonyma drainiert (Craig, Darling, \& Rothney, 1957; Gathman \& Nadas, 1970).

\section{Kardiale Konnektion}

Von einer kardialen Konnektion spricht man, wenn die Lungenvenen direkt in den rechten Vorhof bzw. in den Sinus coronarius münden. (Craig et al., 1957; Gathman \& Nadas, 1970)

\section{Infrakardiale Konnektion}

Bei der infrakardialen Konnektion münden die Lungenvenen in ein intraabdominelles Gefäß. Dabei vereinen sich die Pulmonalvenen erst zu einem Konfluens hinter dem linken Vorhof, von wo aus die Vena verticalis descendens mit dem Ösophagus das Diaphragma passiert, und in die Pfortader bzw. ihre Zuflüsse mündet. Nur selten kommt es zur Mündung in die Vena cava inferior (Craig et al., 1957). Bei diesem Konnektionstyp kommt es nahezu immer zu einer pulmonalvenösen Obstruktion; einerseits durch den Anschluss an das Pfortadersystem, andererseits durch die oftmals bestehende Stenose der Vena verticalis descendens bei Diaphragmapassage (Anderson, 2010).

\section{Gemischte Konnektion}

Bei ca. 5\% der Patienten mit TAPVC drainieren die Lungenvenen nicht in das gleiche Gefäß. Mindestens eine Lungenvene mündet in ein anderes Gefäß, meistens mündet allerdings jede Lungenvene für sich in ein unterschiedliches Gefäß. Dabei sind grundsätzlich alle oben beschriebenen Mündungsstellen möglich, am häufigsten ist allerdings die Mischung aus kardialer und suprakardialer Konnektion (Craig et al., 1957; Gathman \& Nadas, 1970). In manchen Fällen vereinen sich zwei der Lungenvenen bevor sie in ein anderes Gefäß münden; in sehr seltenen Fällen kann es auch vorkommen, dass sich alle Lungenvenen zu einem Gefäß vereinen, wobei dieses Sammelgefäß dann wiederum in unterschiedliche Gefäße drainiert (Choe et al., 1994)

\subsubsection{Pathophysiologie}

Nachdem das oxygenierte Blut der Pulmonalvenen bei totaler Lungenvenenfehlmündung wiederum dem Lungenkreislauf zufließt, ist für das Überleben eine Verbindung zwischen dem Lungenkleinlauf und dem Systemkreislauf (Rechts-Links-Shunt) obligat. Dieser befindet sich nahezu ausschließlich auf Vorhofebene - als offenes Foramen Ovale oder als Vorhofseptumdefekt. Einige Ausnahmen wurden beschrieben, bei denen die Blutmischung auf Ventrikelebene bzw. über den offenen Ductus erfolgte

(Delisle et al., 1976; Hastreiter, Paul, Molthan, \& Miller, 1962). Dieser atriale Shunt erlaubt eine Mischung von oxygeniertem und nicht-oxygeniertem Blut und ist meistens von adäquater Größe (Behrendt, Aberdeen, Waterson, \& Bonham-Carter, 1972; Gathman \& Nadas, 1970). Ist dies nicht der Fall bzw. ist ein solcher bei Geburt nicht vorhanden, muss ein Rash- 
kind-Manöver durchgeführt werden. Dabei wird interventionell ein Vorhofseptumdefekt erzeugt bzw. vergrößert (Rashkind \& Miller, 1966).

Auch wenn dieser Rechts-Links-Shunt ein Überleben ermöglicht, ist dennoch das zirkulierende Blut Mischblut, was bedeutet, dass die Patienten eine arterielle Untersättigung aufweisen, welcher klinisch ggf. als Zyanose erkennbar ist. Je nach Größe und Obstruktion der fehlkonnektierten Lungenvenen, ist der Sauerstoffmangel (und damit die Zyanose) mehr oder weniger stark ausgeprägt. Meist sind Patienten kaum zyanotisch, erst bei starker pulmonalvenöser Obstruktion wird der Sauerstoffmangel klinisch apparent.

Des Weiteren bedingt das rezirkulierende Blut des Lungenkreislaufs eine Rechtsherzbelastung. Der rechte Vorhof ist vergrößert und die Herzwand hypertroph, der linke Vorhof kleiner (Bharati \& Lev, 1973). Der linke Ventrikel wird im Allgemeinen als unverändert beschrieben, auch wenn das linke Herz neben dem oft vergrößerten rechten Herzen klein erscheint (Bove, Geiser, \& Meyer, 1975;

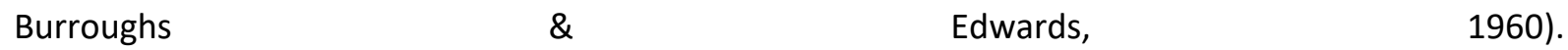
Die Morphologie des rechten Ventrikels ist abhängig vom pulmonalen Flussvolumen, dem Vorhandensein einer pulmonalvenösen Obstrukion und der anatomischen Mündungsstelle der Lungenvenen. So beschreibt Haworth et al. den rechten Ventrikel bei infrakardialer Lungenvenenkonnektion weder als zu groß, noch als hypertroph. Im Gegensatz dazu zeigte sich der rechte Ventrikel bei suprakardialer Konnektion als dilatiert und hypertroph (Haworth \& Reid, 1977). Dies ist dadurch bedingt, dass bei infrakardialem Konnektionstyp aufgrund der meist bestehenden Obstruktion das Rezirkulationsvolumen aus dem Lungenkreislauf nicht stark erhöht ist, wohingegen bei anderen Konnektionstypen ohne Obstruktion das rechte Herz stark Volumen-belastet ist, was letztendlich zu einer Hypertrophie der Muskelzellen und zu einer Dilatation des rechten Herzens führt.

\subsubsection{Pulmonalvenöse Obstruktion}

Eine zusätzliche Obstruktion der fehlmündenden Lungenvenen kann eine schwerwiegende Begleiterscheinung bei diesem Herzfehler sein. Grundsätzlich kann sie bei jeder genannten Konnektionsform auftreten. Bei infrakardialer Konnektion besteht nahezu immer eine pulmonalvenöse Obstruktion (PVO), da hier die Lungenvenen fast ausschließlich in das Pfortadersystem münden und die Leberpassage durch die Sinusoide eine Obstruktion bedingt (Anderson, 2010). Außerdem kommt es bei dem Durchtritt des deszendierenden Sammelgefäßes durch das Diaphragma oft zur Stenosierung des Gefäßes, was auch $\quad \mathrm{zu}$ PVO führt. Meistens entsteht eine pulmonalvenöse Obstruktion bei Stenosen an der Mündungsstelle des pulmonalvenösen Sammelgefäßes in das venöse System des Systemkreislaufs. Bei 65\% der Anschlüsse an die Vena cava superior besteht eine PVO, bei Anschluss an die Vena anonyma in $40 \%$ und bei gemischter Konnektion ebenfalls bei $40 \%$ der Fälle (Delisle et al., 1976). PVO bei kardialer Konnektion ist weitaus seltener, wurde aber bei Mündung in den Sinus coronarius in 20\% der Fälle beschrieben (Jonas, Smolinsky, Mayer, \& Castaneda, 1987). Des Weiteren kann eine PVO durch Kompression des Sammelgefäßes entstehen, wie bereits bei der Diaphragmapassage bei infrakardialer Konnektion beschrieben wurde. Auch bei supradiaphragmaler Konnektion kann es zur Kompression der Vena verticalis kommen, nämlich dann, wenn sie die linke Pulmonalarterie unterkreuzt statt überkreuzt. Der Verlauf dieser Vena Verticalis determiniert also, ob es zu einer Obstruktion kommt oder nicht. Bei einem Verlauf vor der linken Pulmonalarterie, ist eine Obstruktion sehr unwahrscheinlich. Sollte die Vena verticalis allerdings zwischen linker Pulmo- 
nalarterie und linkem Bronchus durchziehen, kommt es meistens zu einer Obstruktion (Anderson, 2010)

Ganz allgemein ist der Durchmesser der Lungenvenen von Bedeutung, da enge und besonders lange Lungenvenen eher zu einer Obstruktion führen, als breitere Auch wenn präoperativ keine pulmonale Obstruktion besteht, korreliert unabhägig von der präoperativen Situation der Durchmesser der Lungenvenen mit der Wahrscheinlichkeit einer postoperativen Obstruktion (Jenkins et al., 1993).

\section{Folgen der PVO}

Bei nicht obstruierter Lungenvenenfehlkonnektion ist aufgrund der Rezirkulation der pulmonale Fluss höher als der systemische, der Druck im rechten Ventrikel und den Lungenarterien zunächst nicht erhöht. Wenn auf Vorhofebene eine ausreichende Mischung des nicht oxygenierten Blutes mit oxygeniertem Blut ist möglich ist, kann eine systemische Sauerstoffsättigung von mehr als $90 \%$ resultieren (Hammon et al., 1980).

Bei pulmonalvenöser Obstruktion mit Anstieg des pulmonalvenösen Druckes kommt es zu einem Lungenödem und konsekutiv auch zu einem Anstieg des pulmonalarteriellen Widerstandes. In schweren Fällen kann dann der Pulmonalisdruck über dem systemischen Blutdruck liegen. Der pulmonale Blutfluss nimmt ab und es kommt zusätzlich zur Volumenbelastung auch zu einer Druckbelastung des rechten Ventrikels (Mathew, Thilenius, Replogle, \& Arcilla, 1977). Eine ausreichende Blutoxygenierung ist dann wegen des Lungenödems und ggfs. reduziertem pulmonalen Blutfluß nicht mehr gewährleistet und die systemische Sauerstoffsättigung kann bis auf $20 \%$ bis $30 \%$ absinken (Anderson). Dies stellt einen absoluten herzchirurgischen Notfall dar, welcher unmittelbar korrektiv behoben werden muss.

\subsubsection{Symptomatik}

Die klinische Symptomatik ist abhängig von dem Bestehen einer PVO. Schon 1962 erkannten Hasreiter et al., dass Vorhandensein und Ausmaß einer PVO die wichtigste Determinante der klinischen Symptomatik ist. Auch wenn diese Beobachtung auf die meisten Patienten zutrifft, können auch symptomarme Patienten eine PVO aufweisen bzw. durch kardiale Dekompensation in kurzer Zeit schwer kranke Patienten werden (Hastreiter et al., 1962).

\subsubsection{Patienten mit PVO}

Patienten mit PVO werden meist in der ersten Lebenswoche durch Zyanose, Tachypnoe und Trinkschwäche auffällig. Diese Symptome sind sehr unspezifisch und können Hinweis sein auf viele Differentialdiagnosen, denen sowohl kardiale als auch pulmonale Prozesse zugrunde liegen können. Auch mögliche fetale Lungenwasserretention und Mekoniumaspiration können sich ähnlich manifestieren (Carter, Capriles, \& Noe, 1969),. Im Unterschied zum Atemnotsyndrom ist der Auskultationsbefund bei Patienten mit TAPVC meist blande; sollten kardiale Herzgeräusche bestehen, sind diese meist nur schwach systolisch zu hören. Bei bereits bestehendem Lungenödem können sich zudem ggf. Rasselgeräusche auskultieren lassen.

Patienten mit PVO sind schwer kranke Neugeborene mit mehr oder wenig stark ausgeprägter Zyanose (Carter et al., 1969). Dabei ist der Zyanosegrad von dem Ausmaß der PVO abhängig. Eine milde Zyanose kann durchaus übersehen werden. 
Nachdem die beschriebene Symptomatik zahlreiche Differentialdiagnosen offen lässt, ist eine unverzügliche echokardiografische Untersuchung unerlässlich.

\subsubsection{Patienten ohne PVO}

Patienten ohne PVO weisen im Regelfall keine Zyanose auf. Oftmals präsentieren sich diese Patienten erst im zweiten oder dritten Lebensmonat mit zunehmender Herzinsuffizienz, deren einziges Symptom eventuell Trinkschwäche ist. Bei diesen Patienten bestimmt der pulmonale Fluss das Ausmaß der Symptomatik. Stark erhöhter pulmonaler Fluss (der unbehandelt bis zur pulmonalen Hypertension führen kann) verursacht Tachypnoe vor allem beim Trinken und längerfristig zu verlangsamter Entwicklung. Wenn der pulmonale Blutfluss nur wenig erhöht ist, kann die Symptomatik so schwach sein, dass solche Patienten Jahre lang klinisch unauffällig bleiben.

\subsubsection{Natürlicher Verlauf}

Die allgemeine, natürliche Prognose für Patienten mit TAPVC ist schlecht. Nur ca. 20\% überleben ohne Operation - das erste Lebensjahr (Burchell, 1956; Burroughs \& Edwards, 1960; Keith et al., 1954). Davon sterben etwa $50 \%$ innerhalb der ersten drei Lebensmonate, in denen sie auffällig werden durch Tachypnoe, Zyanose und Herzinsuffizienz. Dies sind Patienten mit PVO, besonders kleinen Konnektionsgefäßen und Patienten mit kleinem PFO. Von den anderen 50\%, welche die kritischen ersten Lebensmonate überleben, sterben wiederum etwa $50 \%$ innerhalb des ersten Jahres. Patienten, die das erste Jahr erreichen, haben meist einen großen ASD, der für die ersten 10 bis 20 Jahre eine relativ stabile hämodynamische Situation ermöglicht, während der die Patientenen bis auf eine leichte Zyanose, vor allem unter körperlicher Belastung, klinisch unauffällig sind (Gathman \& Nadas, 1970). Erst in der zweiten Dekade kommt es wegen der langen Lungenüberflutung zu Strukturveränderungen des Lungenstromgebietes, welche zur sogenannten Eisenmenger-Reaktion führen, wodurch der Lungenblutfluss abnimmt, und Patienten symptomatisch werden.

\subsubsection{Diagnostik}

Bei Neugeborenen mit Tachypnoe und respiratorischer Insuffizienz mit Verdacht auf schwere PVO ist eine arterielle Blutgasanalyse wegweisend, welche sowohl eine Hypoxie als auch eine metabolische Azidose aufzeigen würde. Dies ist allerdings wenig spezifisch. Auch wenn eine Röntgen-ThoraxAufnahme bereits Auffälligkeiten zeigen kann (z.B. Herzschattenvergrößerung bei erhöhtem rechtsventrikulärem Volumen bzw. Lungenödem, besonders bei PVO) und ggf. andere, pulmonale Ursachen aufzeigen bzw ausschließen kann, ist die Herzechographie die Methode der Wahl zur Diagnostik der TAPVC (Carter et al., 1969). Erste Hinweise sind ein Rechts-Links-Shunt auf Vorhofebene, ein kleiner linker Vorhof oder eine dilatierte Vena cava superior. Ein zusätzlich nicht darstellbarer Anschluss der Pulmonalvenen an den linken Vorhof ist bereits hochverdächtig. Es gilt auch zu unterscheiden, ob vielleicht nur eine partielle Lungenvenenfehlmündung vorliegt, welche aufgrund der günstigeren Pathophysiologie ganz anders zu bewerten wäre. Zur Diagnose sollte sich der Sinus confluens echokardiographisch darstellen lassen. Dies ist laut Smallhorn et al in 91\% der Fälle möglich (Smallhorn et al., 1987). Dieser zeigt sich als echoarmer Raum, hinter dem linken Vorhof gelegen und von diesem deutlich abgetrennt. Zur Unterscheidung von den Pulmonalarterien zeigt sich im Confluens kein pulsatiler Fluss (Patel, Lane, Spector, Smith, \& Crane, 2005; Valsangiacomo, Hornberger, Barrea, Smallhorn, \& Yoo, 2003). 
Zur genauen Typisierung muss anschließend die Konnektion des Confluens an den venösen Systemkreislauf dargestellt werden. Dabei kann es sich um ein absteigendes Gefäß handeln (Vena descendens), welches sich meist am Durchtritt durch das Diaphragma darstellen lässt. Im Gegensatz zur meist dahinter liegenden Aorta, zeigt sich hier in der Doppler-Untersuchung kein pulsatiler Fluss. In diesem Fall handelt es sich um eine TAPVC vom infrakardialen Typ. Zeigt sich ein vertikales Gefäß, welches z.B. in die Vena brachiocephalica mündet, handelt es sich um eine TAPVC vom suprakardialen Typ. Lässt sich kein weiterführendes Gefäß darstellen, dann findet sich der Anschluss meist direkt an den Koronarsinus, bzw. direkt an den rechten Vorhof und zeigt somit eine TAPVC vom kardialen Typ.

Der Untersucher sollte angehalten werden, bereits bei der diagnostischen Untersuchung die Durchmesser aller vier Lungenvenen zwischen Hilum und Konfluens zu messen. Deren Summe zeigte sich als guter prädiktiver Wert für das postoperative outcome (Romero-Cardenas et al., 1991).

Mittlerweile ist die alleinige Echokardiographie Standard zur Diagnose der TAPVC. Bereits 1983 verzichtete man erstmals auf eine diagnostische Herzkatheteruntersuchung (Stark et al., 1983). Die anomale Anatomie der TAPVC kann non-invasiv dargestellt werden und das Vorhandensein einer PVO zeigt sich meist bereits klinisch, ohne invasive Testung (Brown, De Lange, Dyar, Impastato, \& Shirali, 1998; J. K. Wang et al., 1993). Nachdem der Mehrgewinn an Information durch invasive Diagnostik (Herzkatheteruntersuchung und Angiographie) gering ist und die Komplikationen nicht zu verachten sind, erfolgt die Diagnose mittlerweile durch eine Echokardiographie. Insbesondere bei Patienten mit Verdacht auf PVO ist eine invasive Diagnostik selten indiziert; erstens, weil die Passage des Katheters der eventuell vorhandenen Obstruktion diese während der Untersuchungssituation verschlechtert und zweitens weil es durch die Applikation von Kontrastmittel zu einer Exazerbation eines eventuell vorhandenen Lungenödems kommen kann (Volumenbelastung, osmotische Kräfte). (Sano, Brawn, \& Mee, 1989)

\section{Assoziierte Herzfehler (vielleicht zu lang?!)}

Mehr als 5\% aller Neugeborenen mit TAPVC hat zusätzlich eine univentrikuläre Pathologie, und zwar... (Raisher 1992)

\subsection{Behandlung}

\subsubsection{Präoperatives Management}

Das präoperative Management ist stark abhängig von der Schwere der klinischen Symptomatik. An erster Stelle steht die korrekte Diagnostik. Wegen der zahlreichen Differentialdiagnosen beim kranken, tachypnoeischen Neugeboren sollte hier die Indikation zur Echokardiographie großzügig gestellt werden. Meist ist diese dann bereits beweisend für das Vorliegen einer TAPVC.

Ist die TAPVC bestätigt, ist die Behandlung erster Wahl immer die korrigierende Operation. In vielen Fällen muß die Operation schon im Neugeborenenalter durchgeführt werden. Je nach Schwere der Symptomatik (Lungenödem, Azidose, pulmonale Hypertension etc) sind eventuell bereits vor der Operation eine Intubation und Beatmung und weitere intensivmedizinische Maßnahmen nötig. Bei kritisch kranken Neugeborenen mit PVO sollte eine notfallmäßige Korrekturoperation angestrebt werden. 


\subsubsection{Korrekturoperation}

Grundsätzlich ist das Ziel der Korrektur, aus den zwei anomal parallel geschalteten Lungen- und Systemkreisläufen zwei in Serie geschaltete Kreisläufe herzustellen. Nachdem bei der TAPVC die Lungenvenen mit oxygeniertem Blut an das venöse System (und damit wieder der Lunge zuführendes System) anschließen, gilt es, diesen Anschluss zu unterbinden und einen physiologisch korrekten Anschluss an den linken Vorhof herzustellen. Der Septumdefekt auf Vorhofebene wird dabei auch verschlossen und das pulmonalvenöse Sammelgefäß in den meisten Fällen ligiert. Die Operation wird am kardiopulmonalen Bypass durchgeführt: Eröffnung des Brustkorbes mittels medianer Sternotomie, Präparation der zuführenden venösen und abführenden arteriellen Gefäße, Inzision und Setzen von zirkulären Nähten am rechten Vorhof oder an oberer und unterer Hohlvene, und Einführen der ableitenden Kanülen der Herz-Lungen-Maschine, Inzision und Setzen von Nähten an der Aorta ascendens zum Einführen der rückführenden Kanüle der Herz-Lungen-Maschine. Während der Operation wird das Herz mittels kardioplegischer Lösung "stillgelegt". Das Blut und damit der Körper wird durch die Herz-Lungen-Maschine gekühlt, um die Hypoxie-Toleranz zu erhöhen.

Nach der Herstellung der extrakorporalen Zirkulation variieren die Operationsschritte je nach Konnektionstyp, wie unten beschrieben. Prinzipiell gilt es aber immer, den Konfluens, in den die Pulmonalvenen zusammenfließen, mit dem linken Vorhof zu anastomosieren und das abführende Gefäß des Konfluens zu ligieren.

Nachdem die Anastomose und damit Korrektur erfolgt ist, wird das Herz sorgfältig entlüftet, reperfundiert, die extrakorporale Zirkulation beendet, die zentralvenösen und aortale Kanülen entfernt und der Thorax verschlossen.

\subsubsection{Korrektur einer suprakardialen TAPVC Konnektion}

Wie bereits oben beschrieben, besteht bei suprakardialer Konnektion ein pulmonalvenöser Konfluens über den das Blut über ein weiterleitendes Gefäß (z.B. V. verticalis) in eine Vene des Systemkreislaufs mündet. Dies ist meist die Vena anonyma, gelegentlich auch die V. cava superior, kann aber auch in Einzelfällen ein anderes venöses Gefäß sein (z.B. V. brachiocephalica).

Der Konfluens liegt typischerweise hinter dem linken Vorhof retroperikardial. Bevor eine Anastomose erfolgen kann, müssen alle Strukturen dargestellt werden und das Perikardium eingeschnitten werden. Dazu kann es nötig sein das Herz bzw. Herzapex zu lösen und anzuheben, um die retrokardial gelegenen Strukturen einsehen zu können.

Zur Herstellung der Anastomose zwischen Konfluens und linkem Vorhof gibt es zwei Möglichkeiten. Das Verbindungsgefäß zwischen Konfluens und der Vene des Systemkreislaufs wird ligiert. Anschließend wir der Konfluens mobilisiert und inzidiert; ebenso erfolgt eine Inzision der posterioren Vorhofwand von "außen" und eine Anastomose zwischen Konfluens und Vorhof wird hergestellt. Nun gilt es, die dazu nötigen Nähte nicht zu eng zu setzen, da sonst postoperative Stenosen entstehen können. Andererseits muss ein postoperativer Blutverlust an der Anastomose verhindert werden. Anschließend wird durch eine Inzision im rechten Vorhof der Vorhofseptumdefekt verschlossen und die Inzision des rechten Vorhofs wieder verschlossen. In einer anderen Herangehensweise wird ein Zugang zur Hinterwand des linken Vorhofs durch das 
Foramen ovale gewählt. Hierzu erfolgt die Eröffnung des rechten Vorhofs parallel zum Sulcus coronarius. Durch einen Einschnitt des Foramen ovale wird der linke Vorhof erreicht und die somit einsehbare Hinterwand längs inzidiert. Darunter kommt der Konfluens zum Vorschein, welche ebenso eingeschnitten wird. Anschließend werden die Ränder adaptiert und somit eine Anastomose hergestellt. Anschließend wird der Vorhofseptumdefekt verschlossen; meist mittels Patch, um gleichzeitig eine Vergrößerung des meist unterentwickelten linken Vorhofs vorzunehmen. Bei dieser Vorgehensweise müssen die beteiligten Strukturen nicht mobilisiert werden, was auch bedeutet, dass sich die Anastomose nicht "verziehen" kann, nachdem sowohl Konfluens als auch Herz in ihrer ursprünglichen Position belassen werden.

Zur Herstellung der Anastomose werden unterschiedliche Nahtmaterialien verwendet; resorbierbare und nicht resorbierbare (siehe Diskussion).

\subsubsection{Korrektion der kardialen TAPVC Konnektion}

Prinzip dieser Operation ist eine „Umleitung" des in den rechten Vorhof mündenden Blutes in den linken Vorhof. Anomale Mündungsstelle ist entweder der Koronarsinus oder direkt der rechte Vorhof.

Münden die Lungenvenen in den Koronarsinus, ist der entscheidende operative Schritt die Inzision des Daches des Koronarsinus sowie die Resektion des Vorhofseptum, sodass der Koronarsinus direkt in den linken Vorhof drainiert. Dabei muss darauf geachtet werden, weder AV-Knoten noch leitende Strukturen zu beschädigen. Anschließend wird eine Neuseptierung der Vorhöfe vorgenommen, wobei man den geschaffenen Defekt mit einem großen Patch (z.B. Perikard) verschließt, sodass das Blut des „entdeckelten“ Koronarsinus in den linken Vorhof fließt. Anders ausgedrückt ist dann der linke Vorhof um den Koronarsinus erweitert; erst durch Resektion des Daches des Koronarsinus und des Septums und anschließend Neuseptierung der Vorhöfe.

\subsubsection{Korrektur der TAPVC vom infrakardialen Typ}

Hier vereinen sich die Pulmonalvenen ebenfalls hinter dem linken Vorhof $Y$ - oder T-förmig zu einer Vena verticalis descendens, welche das Diaphragma passiert und in eine abdominelle Vene mündet. Auch hier besteht an dem Y- bzw T-förmigen Zusammenfluss eine Art Konfluens, welcher nun Anschluss finden soll an den linken Vorhof. Auch hier können beide Zugangswege verwendet werden; entweder durch Hervorrotieren des Herzens direkt von hinten, oder intrakardial durch beide Vorhöfe (über eine Inzision des rechten Vorhofs mit anschließendem Zugang zum linken Vorhof über das Foramen ovale). Gemeinsam sind diesen zwei Wegen folgende Schritte: Ligatur der Vena verticalis descendens, distal der Mündung aller Pulmonalvenen. Als nächstes erfolgt die Identifizierung und Präparation des Konfluens. Anschließend werden die Vorderwand des Konfluens, sowie die Hinterwand des linken Vorhofes eingeschnitten und miteinander anastomosiert. Dieser Schritt kann wie oben erklärt über zwei unterschiedliche Zugänge vorgenommen werden. Nachdem der Konfluens meist länglich ist (T- oder Y-förmig), ist meist ein etwas längerer Schnitt nötig. Anschließend wird der Konfluens distal ligiert und ggf. auch abgesetzt, um eine geschmeidigere Form der Anastomose zu gewährleisten. Der weitere Operationsverlauf ist gleich, wie bei den anderen Konnektionstypen. 


\subsubsection{Postoperative Komplikationen}

\subsubsection{Linksatriale Dekompensation}

Durch die operative Korrektur kommt es zu relevanten Änderungen der Hämodynamik. Einerseits werden der rechte Ventrikel und die Lungenstrombahn entlastet, andererseits kommt es zu einer Volumen-Mehrbelastung des linken Ventrikels, welcher präoperativ oft unterentwickelt ist, nachdem sein Blutzufluß vor Korrektur lediglich durch einen oftmals kleinen Vorhofseptumdefekt stattfindet. Sollte der linke Ventrikel sich intraoperativ bereits als hypoplastisch erweisen, kann ein kleiner Vorhofseptumdefekt belassen werden als Art „Überlaufventil“, über das sich die Vorlast des linken Herzens regulieren kann. Dieser verschließt sich entweder spontan oder später interventionell verschlossen werden.

\subsubsection{Pulmonale Hypertension}

Bei vielen Patienten, insbesondere bei präoperativer PVO, kommt es innerhalb der ersten 3 bis 5 Tage postoperativ zu einer krisenhaften Anstieg des pulmonalarteriellen Druckes, selbst wenn die Obstruktion chirurgisch behoben wurde. Deshalb wird der pulmonalarterielle Druck gelegentlich invasiv gemessen. Deswegen kann eine verlängerte Sedierung und Beatmung zur Vermeidung von pulmonalen Krisen bzw. von Hyperkapnie oder Hypöxamie indiziert sein. Auch hier kann das Belassen einer Vorhoflücke im Falle eines Rechtsherzversagens im Rahmen pulmonaler Krisen vorteilhaft sein.

\subsubsection{Pulmonalvenöse Obstruktion}

Eine gefürchtete Komplikation ist die postoperative pulmonalvenöse Obstruktion, da diese mit einer schlechten Prognose einhergeht. Die PVO kann konsekutiv zu einer Druckbelastung des rechten Ventrikels führen. Am häufigsten tritt diese bei Patienten auf, welche bereits präoperativ eine Obstruktion mit Lungenstauung aufwiesen. Solche Patienten benötigen oft eine verlängerte und invasiveRespiratortherapie, in schweren Fällen ist eine extrakorporale Membranoxygenierung (ECMO) notwendig. Aber auch eine Enge der chirurgisch angelegten Anastomose kann zu postoperativer PVO führen. In diesem Fall kann ein Folgeeingriff notwendig sein. Eine Obstruktion kann residuell oder chirurgisch bedingt sein und sich dann bereits unmittelbar nach Korrektur bemerkbar machen, sie kann sich aber auch erst im Laufe von Monaten neu entwickeln. Oft entstehen solche Obstruktionen nicht an der Stelle der ursprünglichen Anastomose, sondern proximal derselben. Postoperativ entstandene Obstruktion der anastomosierten Lungenvenen kann somit Grund für die Notwendigkeit einer neuerlichen Operation sein. Dies ist auch ein Gegenstand der im Folgenden beschriebenen Untersuchung.

\subsubsection{Postoperative Rhythmusstörungen}

Nachdem im Rahmen der Operation das Reizleitungssystem des Herzens gereizt bzw. geschädigt werden kann, zählen postoperative Rhythmusstörungen zu bedrohlichen Komplikationen nach Herzoperationen. Bei meist kontinuierlichem EKG-Monitoring unmittelbar postoperativ wurden supraventrikuläre Tachykardien, Bradyarrhythmien sowie polymonorphe ventrikuläre und supraventrikuläre Extrasystolen verzeichnet (Saxena et al., 1991). Manche Autoren beschreiben eine relativ hohe Inzidenz an bleibenden Sinusknotendysfunktionen; knapp 30\% der Patienten, bei denen eine TAPVC korrigert wurde, zeigen unter Belastung ein mangelndes chronotropes Anpassungsvermögen, ohne 
allerdings signifikante Arrhythmien aufzuweisen (Tanel et al., 2007). Auch wurden Fälle von plötzlichem Herztod innerhalb der ersten sechs postoperativen Wochen dokumentiert, welche durch Sinusknotendysfunktionen erklärt wurden (Byrum, Dick, Behrendt, \& Rosenthal, 1982). Dies wird vereinzelt auch noch Jahre nach Operation beschrieben (Gomes, Feldt, McGoon, \& Danielson, 1970).

\subsubsection{Todesursachen}

Alle genannten früh-postoperativen Komplikationen, wie Linksherzversagen, pulmonal-hypertensive Krise mit Rechtsherzversagen, Lungenödem und Rhythmusstörungen können schließlich zum unmittelbaren Tod oder weiteren intensivmedizinischen Komplikationen, Multiorganversagen und Tod führen.

\subsection{Historische Wandlung der Operationstechnik}

Erstbeschreiber der TAPVC war Wilson im Jahre 1798 (Wilson). Erst 1951 gelang es Muller eine palliative Anastomose zwischen dem linken Vorhof und dem Konfluens herzustellen, ohne allerdings eine Ligatur des Konfluens vorzunehmen (Muller, 1951). Die erste Korrektur unter Hypothermie gelang Lewis im Jahre 1956. Die erste erfolgreiche Korrektur mit kardiopulmonalem Bypass wurde noch im selben Jahr von Burroughs und Kirklin durchgeführt. Erst 1971 zeigten Barrat-Boyes et al., dass eine zusätzliche tiefe Hypothermie bei Kreislaufstillstand bessere Operationserfolge zeigte, insbesondere bei Neugeborenen mit schlechter präoperativer Ausgangslage bei obstruierter TAPVC (BarrattBoyes, Simpson, \& Neutze, 1971).

Im Verlauf besserten sich die Operationsergebnisse drastisch, durch zunehmende Erfahrung mit Operationstechniken, Weiterentwicklung der Techniken der extrakorporalen Zirkulation und der Intensivmedizin. Mit Abnahme der Operationsletalität traten auch Fragen der postoperativen Lebensqualität und psychomotorischen Entwicklung des Kindes in den Vordergrund. Diesbezüglich wurden und werden weiterhin - unter anderem auch Unterschiede im Bypass-Management diskutiert (siehe Diskussion).

Historische Wandlungen bezüglich der chirurgischen Vorgehensweise sind oft Klinik-spezifisch und lassen sich nicht verallgemeinern. Am Deutschen Herzzentrum München zeigte sich in den 1990er Jahren ein Wandel im operativen Zugang weg vom intrakardialen Zugang und hin zum „extrakardialen“ (s.o.). Eine Ära-spezifische Änderung in den operativen Ergebnissen konnte allerdings nicht verzeichnet werden, womit weiterhin beide Methoden als ebenbürtig angesehen werden. 


\section{Methoden}

Erstes Ziel der vorgelegten Untersuchung ist die Identifizierung von Risikofaktoren für postoperative Letalität und für nötige Re-Operationen im gesamten Patientengut des Deutschen Herzzentrums München von 1974 bis 2012.

In zweiter Linie galt es, den möglichen Einfluß von anatomischen und klinischen Faktoren zu analysieren, und zu evaluieren, ob Änderungen von Strategie der extrakorporalen Zirkulation oder in der Verwendung von Nahtmaterial einen Einfluß auf das Überleben oder die Freiheit von Re-Operation hatten.

Um eine akkurate Beschreibung des Patientenkollektives zu erhalten, die im Anschluss eine fundierte Aussage ermöglicht, wurden retrospektive und prospektive Patientendaten erhoben. Diese beschreiben die demographische Verteilung, die präoperative Ausgangssituation, den operativen und unmittelbar postoperativen Verlauf in der Klinik, eventuelle Re-Interventionen und die Situation der Patienten jetzt. Anhand dieser Daten wird es möglich, Risikofaktoren für die Notwendigkeit von ReInterventionen bzw. für verfrühtes Ableben fest zu machen. Diese werden anschließend in der Diskussion interpretiert und bewertet, und mit internationalen Publikationen verglichen.

\subsection{Erfasste Daten}

Der Datensatz setzt sich zusammen aus Daten von insgesamt 193 Patienten. Dabei wurden alle Patienten, die am Deutschen Herzzentrum von 1974 bis 2012 korrigierend an einer TAPVC operiert wurden, eingeschlossen.

In der Untersuchung wurden auch solche Patienten mit einbezogen, die zusätzlich zu der TAPVC andere kardiale Fehlbildungen aufweisen. Ebenso miteinbezogen wurden Patienten, die nach Korrekturoperation am Deutschen Herzzentrum in anderen Zentren weiterbetreut wurden; allerdings nicht solche, deren TAPVC-Korrektur in einem anderen Zentrum erfolgte, zur Nachsorge aber am Deutschen Herzzentrum betreut werden.

\subsection{Retrospektive Daten}

\subsubsection{Demographische Daten}

Von allen Patienten wurde Geschlecht, Gewicht und Größe und Alter bei Operation retrospektiv erhoben. Anhand des Alters zum Operationszeitpunkt wurden zwei Gruppen unterschieden: solche die im Neugeborenenalter und solche, die danach operiert wurden. 


\subsubsection{Präoperative Situation}

Das Augenmerk bei der retrospektiven Analyse der präoperativen Situation galt neben der genauen Diagnose TAPVC auch den zusätzlichen kardialen Diagnosen, dem Vorhandensein präoperativer Stenosen der Lungenvenen und eventuell durchgeführten Vorbehandlungen. Informationen hierzu stammen aus echokardiographischen Befunden, Arztbriefen aus zuweisenden Zentren und Arztbriefen aus dem Deutschen Herzzentrum.

Die Diagnose TAPVC wurde sonographisch gestellt und unterteilt das Patientengut je nach Mündungsstelle der Lungenvenen in vier Gruppen:

- TAPVC vom suprakardialen Typ

- TAPVC vom kardialen Typ

- TAPVC vom infrakardialen Typ

- TAPVC vom gemischten Typ

Des Weiteren wurde anhand der Echobefunde (ggf. ergänzt durch eine Herzkatheteruntersuchung) unterschieden zwischen Patienten, deren Lungenvenen bereits vor der Korrekturoperation eine Obstruktion zeigen und solchen, deren Lungenvenen der restlichen Anatomie entsprechende Lumenverhältnisse aufweisen und somit initial nicht obstruiert sind. Präoperative Obstruktionen zeigen sich besonders bei Patienten mit einer TAPVC vom infrakardialen Typ, bei denen das Sammelgefäß in die Vena portae mündet. Außerdem fanden sich unter den Patienten einige, die neben der TAPVC zusätzliche kardiale Diagnosen aufwiesen, und somit zur Kategorie „komplexer Herzfehler" gehören. Vordergründig sind hier die Diagnosen Single Ventricle und Heterotaxie-Syndrom. Extrakardiale Fehlbildungen wurden nicht gewertet. Auch Ductuspersistenz und ASDs wurden nicht als assoziierter Herzfehler gezählt, da sie streng genommen Teil der TAPVC sind und ein Überleben erst ermöglichen. Der zusätzliche Nachweis einer pulmonalen Hypertonie wurde in die Datensammlung miteinbezogen und wird in der Diskussion anschließend besprochen.

Bei einem Teil der Patienten wurde vor der Korrektur der TAPVC ein interventioneller oder herzchirurgischer Eingriff durchgeführt und im Folgenden als „Voroperation“ bezeichnet. Auch Eingriffe, die an anderen Krankenhäusern vorgenommen wurden, wurden hierbei berücksichtigt. Diese waren größtenteils Manöver nach Rashkind.

\subsubsection{OP-Daten}

Anhand der OP-Protokolle aus dem Archiv des Deutschen Herzzentrums München wurden für alle Patienten nach dem gleichen Schema Daten gesammelt:

- OP-Zeiten (Dauer der Operation, Bypass-Zeit, Aortenklemmzeit und Zeit des Kreislaufstillstandes, wenn im totalen Kreislaufstillstand operiert wurde)

- niedrigste Körpertemperatur während der EKZ

- Art der kardioplegischen Lösung (wenn verwendet) 
- verwendetes Nahtmaterial für die Anastomose, wobei unterschieden wurde in resorbierbares und nicht resorbierbares Nahtmaterial.

Auch der stationäre Verlauf im Anschluss an die Korrekturoperation wurde recherchiert. Nach Angabe der Protokolle der nachsorgenden Intensivstation wurden Beatmungsdauer im Anschluss an die OP (in Tagen), Komplikationen und Dauer des Krankenhausaufenthaltes (in Tagen) festgehalten.

\subsubsection{OP-Zeitraum}

Im Jahr 1990 wurde der operative Zugangsweg zur Anastomosierung von linkem Vorhof und pulmonalvenösem Sammelgefäß geändert. Vor 1990 wurde der rechte Vorhof inzidiert, ein Schnitt über das Septum gesetzt und somit Zugang zum linken Vorhof geschaffen. Durch diesen Zugang wurde die Hinterwand des linken Vorhofs längs inzidiert, und mit dem darunter liegenden Konfluens der Pulmonalvenen anastomosiert. Nach 1990 begann man das gesamte Herz zu luxieren um somit einen direkt Zugang zum linken Vorhof und zum pulmonalvenösen Sammelgefäß zu erhalten.

Um Unterschiede in Letalitäts- und Re-Operationswahrscheinlichkeiten festzustellen, wurde das Patientenkollektiv in zwei Gruppen unterteilt: solche, die vor 1990 operiert wurden und solche, die nach 1990 operiert wurden.

\subsection{Prospektive Daten / Follow-up}

Um die Langzeitergebnisse nach Korrektur bewerten zu können, ist in der prospektiven Datensammlung ein genaues und möglichst detailliertes Follow-up unerlässlich. Als Follow - up Zeit wird die Zeit zwischen der Korrekturoperation und dem Zeitpunkt der Datenerhebung (Jahr 2012) bzw. die Zeit zwischen Korrekturoperation und Todesdatum bezeichnet. Sollten in diesem Zeitraum weitere Operationen vollzogen worden sein, so wurden diese als Re-OPs vermerkt und im Kapitel "ReOperationen" separat behandelt.

Bei der Erhebung der prospektiven Daten waren folgende Fragen von besonderem Interesse:

> Mussten Re-Operationen oder Interventionen durchgeführt werden? Wenn ja, warum und wie lange nach der Korrektur-OP?

Wie geht es den Patienten heute? Wie viele sind verstorben? Was waren die Todesursachen?

Um auf diese Fragen Antworten zu beantworten, wurden Informationen aus Krankenakten des Deutschen Herzzentrums, Briefe von anderen behandelnden Ärzten , Informationen von Hausärzten, Familienmitgliedern und Meldeämtern gesammelt; teils schriftlich, teils telefonisch. Aus diesem doch umfangreichen Informationspool war es möglich, ein genaues Follow-up für jeden einzelnen Patienten zu erstellen. Im Anschluss werden diese dargestellt und diskutiert.

\subsubsection{Vitalstatus}

Von allen Patienten wurde der Vitalstatus über Telefonate und Korrespondenz mit Hausärzten, Familienmitgliedern und Meldeämtern erhoben. Aus diesen Daten wurden Überlebenskurven nach Kaplan Meier erstellt. Dabei wurde die gesamte Überlebenswahrscheinlichkeit (für alle Patienten), die Überlebenswahrscheinlichkeit für Patienten mit isolierter TAPVC und die Überlebenswahrscheinlich- 
keit für Patienten mit zusätzlichen kardialen Anomalien separat berechnet.

\subsubsection{Re-Operationen}

Als Re-Operationen wurden alle späteren Eingriffe am Herzen gewertet, unabhängig davon in welcher Klinik sie durchgeführt wurden. Dazu zählen auch Eingriffe am Herzen, die unabhängig von der Korrektur der TAPVC vorgenommen wurden; also Operationen wegen anderer Diagnosen. Kleine Eingriffe wie sekundärer Thoraxverschluss oder Blutstillung während des stationären Aufenthaltes im Anschluss an die Korrekturoperation wurden nicht als Re-Operation gezählt. Interventionelle Eingriffe am Herzen wurden als Re-Interventionen ebenfalls zu den Re-Operationen gezählt, allerdings wurde nur bei einem Patienten eine interventionelle Maßnahme durchgeführt. Schrittmacherimplantationen, Radiofrequenzablationen des Vorhofs und Herzkatheteruntersuchungen zu diagnostischen Zwecken wurden nicht als Re-Intervention gewertet. Auch Zeitpunkt der Re-OP und Abstand zur Korrektur-OP wurden festgehalten. In der Berechnung der Freiheit von Re-OPs wurde im Falle von Re-OPs das Datum der ersten Re-OP verwendet.

Nachdem für die Bewertung der Langzeitergebnisse der Korrektur der TAPVC vor allem die Frage nach Re-Operationen an den Anastomosen der Lungenvenen mit dem rechten Atrium von besonderer Bedeutung sind, wurden die Patienten in zwei Gruppen unterteilt:

- Re-OP an Lungenvenenanastomose

- Sonstige kardiale Re-OPs

Diese Unterscheidung wird in der Auswertung berücksichtigt; in der Diskussion werden mögliche Gründe für Re-Operationen ausführlich besprochen.

Des Weiteren wurde eine Risikoanalyse durchgeführt, um mögliche Faktoren zu isolieren, die die Notwendigkeit einer Re-OP wahrscheinlicher machen. Dabei wurden alle erhobenen Parameter (Alter bei OP, Geschlecht, TAPVC-Typ, präoperativ bestehende Obstruktion, zusätzliche kardiale Diagnosen wie Single ventricle und Heterotaxie-Syndrom, pulmonale Hypertonie, Nahtmaterial und Kreislaufstillstand) auf statistische Relevanz für den Endpunkt „Reoperation“ getestet.

\subsubsection{Verstorbene Patienten}

Bei den verstorbenen Patienten wurde mittels Patientenakten und Unterlagen von Hausärzten bzw. zuletzt behandelnder Ärzte die Todesursache ermittelt.

Hier werden zwei große Gruppen unterteilt:

1. Patienten, die während ihres stationären Aufenthaltes zur Korrekturoperation verstarben (Frühletalität)

2. Patienten, die nach Entlassung verstarben (Spätletalität) In dieser Gruppe wurde nochmals eine zeitliche Streuung erkannt und aufgezeigt

\subsection{Statistische Auswertung}

Allgemeine Vorgehensweise 
Alle oben beschriebenen Patientendaten (s. Liste unten) wurden tabellarisch erfasst. Dabei wurden Daten wie Sonografie-Befunde zur präoperativen Diagnostik in Klassen unterteilt (z.B. präoperative Obstruktion ja/nein) und zur statistischen Auswertung codiert. Kontinuierliche Variablen, wie z.B. Alter bei Korrektur oder Korrektur-Zeitpunkt wurden an einem klinisch relevanten Cut-Off Punkt dichotomisiert (z.B. 30 Lebenstage als Ende der Neugeborenenperiode oder vor/nach 1990, dem Jahr der Einführung neuer OP-Techniken im DHM), wodurch sie als kategorische Variablen in die statische Auswertung mit einbezogen werden konnten.

Die Wahrscheinlichkeiten wurden nach der Kaplan-Meier-Methode berechnet. Zur Berechnung der Freiheit-von-Events-Kurven wurde der Zeitpunkt der operativen Korrektur der TAPVC zum Zeitpunkt Null erklärt. Anschließend wurden die Kurven mittels log-rank Test verglichen. Mittels CoxRegressionsanalyse wurde die Überlebenswahrscheinlichkeit modelliert und als Ausfallsraten (hazard ratio) angegeben. Zur Analyse potentieller Risikofaktoren wurden folgende Endpunkte definiert: Tod, kardiale Re-Operationen, Re-Operationen bei PVO. Als potentielle Einflussfaktoren auf die genannten Endpunkte wurden folgende Parameter in der univariaten Analyse überprüft:

- Männliches Geschlecht

- Alter bei Korrektur-OP <30 Tage

- Korrektur vor dem Jahre 1990

- suprakardiale TAPVC, kardiale TAPVC, infrakardiale TAPVC, gemischte TAPVC

- PVO vor Korrektur

- komplexe Anomalien

- assoziierte univentrikuläre Malformationen (als Extragruppe)

- Heterotaxie

- totaler Kreislaufstillstand in tiefer Hypothermie (DHCA) während Korrektur-OP

- pulmonale Hypertension

- nicht-absorbierbares Nahtmaterial.

Alle Variablen, die in der univariaten Analyse einen $p$-Wert $<0,5$ zeigten, wurden in die anschließende multivariate Analyse miteinbezogen.

Diese Auswertung/Analyse erfolgte gesondert für drei unterschiedliche Kollektive:

1) alle Patienten

2) Patienten mit isolierter TAPVC

3) Patienten mit komplexer TAPVC.

Die gesamte statische Auswertung erfolge mit dem Statistikprogramm SPSS 20.0 für Windows (SPSS Inc., Chicago, IL, USA). 


\subsection{Methodenkritik}

\subsubsection{Aufbau der Studie}

Nachdem es sich hier um eine retrospektive Studie handelt, war es nicht möglich, auf die Art und Weise und Qualität der Dokumentation Einfluss zu nehmen. Das bedeutet, dass im Nachhinein interessante Fragestellungen (z.B. Operationsdetails) nicht mehr beantwortet werden können. Auch war festzustellen, dass sich die Genauigkeit der Datenfesthaltung über die Jahre geändert hat. OPBerichte aus früheren Jahren zum Beispiel waren meist weniger umfassend als rezentere. Der lange Beobachtungszeitraum, die unterschiedlichen Methoden und Qualität der Dokumentation (z.B. durch Einführung großer klinikinterner Datenbanken, welche wiederum detailgenauere Daten erfassen) und die große Zahl der beteiligten Personen, der Wandel und die Weiterentwicklung der diagnostischen Möglichkeiten usw. machen eine Homogenität der Daten für das ganze Patientenkollektiv unmöglich.

Bei der Erhebung der prospektiven Daten (Follow-up) musste oft auf Unterlagen anderer Kliniken und Ärzte zurückgegriffen werden, die in ihrer Detailliertheit erhebliche Schwankungen aufwiesen. Oftmals blieb ein telefonisches Nachforschen der einzige Weg, relevante Informationen zu erhalten, wobei der Umfang der gelieferten Informationen stark von der befragten Person abhing. Auch konnte die Gültigkeit dieser Informationen nicht genauer überprüft werden.

\subsubsection{Datenverlust}

Lückenhafte Dokumentation, fehlende Unterlagen und Unlesbarkeit mancher Schriftstücke führten trotz intensiven Nachforschens zu einem gewissen Datenschwund, weswegen es nicht möglich war, von allen Patienten einen vollständigen Datensatz zu bekommen. Das Follow-up war zu 95\% komplett. Von den insgesamt 193 Patienten konnte von 184 der Verbleib nachverfolgt werden. Von neun Patienten konnten keine Informationen zum Verbleib ausfindig gemacht werden, was bei acht Patienten durch Wegzug ins Ausland bedingt war, der nicht durch dortige Meldeämter nachvollzogen werden konnte. Von diesen neun Patienten konnten trotzdem Aussagen zum Follow-up über einen Zeitraum von zwei Wochen bis 21 Jahren gemacht werden. Betrachtet man das Gesamtkollektiv, wurden Informationen zu 2902 Patientenjahren gesammelt. 


\section{Ergebnisse}

\subsection{Präoperative Daten}

\subsubsection{Demographische Daten}

Bei 193 Patienten wurde zwischen 1974 und 2011 am Deutschen Herzzentrum eine TAPVC korrigierend operiert. Davon waren 85 weiblich (44\%) und 108 männlich (56\%). Dabei handelt es sich sowohl um Patienten, die eine TAPVC als einzigen Herzfehler aufwiesen, als auch solche, die zusätzliche kardiale Anomalien hatten. Betrachtet man die Untergruppe der Patienten, die eine zusätzliche Anomalie aufwiesen separat, fällt auf, dass von den insgesamt 16 Patienten 11 (68,8\%) weiblich waren und 5 $(31,2 \%)$ männlich.

\subsubsection{TAPVC - Typen}

Verteilung der verschiedenen Typen von TAPVC:

\begin{tabular}{lcl}
\hline TAPVC-Typ & Anzahl & Prozent \\
\hline suprakardial & 85 & $45,0 \%$ \\
\hline kardial & 34 & $18,0 \%$ \\
\hline infrakardial & 59 & $31,2 \%$ \\
\hline gemischt & 11 & $5,8 \%$ \\
\hline
\end{tabular}

Tabelle 1 Häufigkeitsverteilung der einzelnen TAPVC Typen im Patientenkollektiv

\subsubsection{Obstruktion}

Eine schon vor Operation bestehende Obstruktion der Lungenvenen wurde bei 105 (54\%) Patienten festgestellt. Dies entspricht 54\% des Patientenkollektivs.

Von einer präoperativen Obstruktion waren größtenteils Patienten betroffen, die eine TAPVC vom infrakardialen Typ haben (56\% derer mit Obstruktion, 31\% des gesamten Patientengutes). 


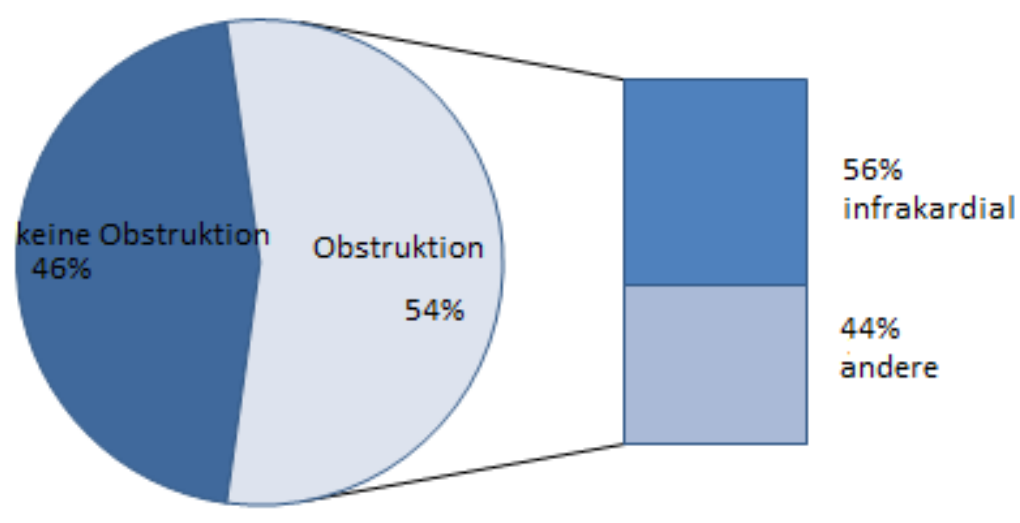

Diagramm 1 Patienten mit und ohne präoperativer PVO, seitlich TAPVC-Typen der Patienten, mit PVO

\begin{tabular}{lc}
\hline Patienten ohne Obstruktion & 88 \\
Patienten mit Obstruktion & 105 \\
- davon infrakardialer Typ & 59 \\
- davon suprakardialer Typ & 18 \\
- davon kardialer Typ & 1 \\
- davon gemischter Typ & 1 \\
\hline
\end{tabular}

Tabelle 2 Anzahl der Patienten mit PVO und Aufschlüsselung der jeweiligen TAPVC-Typen

Wie Diagramm 2 zeigt, hatten 59 Patienten der insgesamt 105 Patienten mit Obstruktion, eine TAPVC vom infrakardialen Typ. Von den übrigen 20 Patienten, haben 18 eine TAPVC vom suprakardialen Typ, ein Patient eine TAPVC vom kardialen und einer vom gemischten Typ. Daraus gibt sich die in Tabelle gezeigte Verteilung der TAPVC Typen innerhalb der Patienten, die eine präoperative Obstruktion aufweisen.

Alle Patienten mit TAPVC vom infrakardialen Typ zeigten präoperativ eine pulmonalvenöse Obstruktion:

\begin{tabular}{|ll|}
\hline TAPVC Typ & Anzahl Patienten mit PVO \\
\hline infrakardial & 59 von $59(100 \%)$ \\
\hline suprakardial & 18 von $85(21,2 \%)$ \\
\hline kardial & 1 von $34(2,9 \%)$ \\
\hline gemischt & 1 von $11(9,1 \%)$ \\
\hline
\end{tabular}

Tabelle 3 Anteil an Patienten mit PVO je nach TAPVC-Typ

\subsubsection{Assoziierte Herzfehler und pulmonale Hypertonie}

16 der insgesamt 193 Patienten (8,3\%) wiesen assoziierte Fehlbildungen am Herzen auf und bilden somit die Gruppe der Patienten mit einem komplexen Herzfehler. 
Von den insgesamt 16 Patienten hatten elf eine univentrikuläre Malformation und sieben ein Heterotaxie-Syndrom; sechs der 7 Patienten mit Heterotaxie zählen ebenfalls zum Kollektiv mit univentrikulärer Malformation

\begin{tabular}{|c|c|c|c|c|}
\hline \multicolumn{2}{|c|}{ Assoziierte Fehlbildung } & Anzahl Patienten & \multicolumn{2}{|c|}{$\begin{array}{l}\text { Andere zUsatzllcne Herztenler WIe VSU, } \\
\text { TGA und Pulmonalklappenstenosen waren }\end{array}$} \\
\hline \multirow{2}{*}{\multicolumn{2}{|c|}{$\begin{array}{l}\text { Univentrikuläre Malformation } \\
\text { davon zusätzl. Heterotaxie }\end{array}$}} & & \multirow{4}{*}{\multicolumn{2}{|c|}{$\begin{array}{l}\text { Einzelfälle. Bei nur einem dieser Patienter } \\
\text { wurde vor der Korrekturoperation ein€ } \\
\text { Korrektur der assoziierten Fehlbildung } \\
\text { (Pulmonalklappenstenose) durchgeführt. }\end{array}$}} \\
\hline & & 6 & & \\
\hline \multicolumn{2}{|c|}{ Heterotaxie, isoliert } & 1 & & \\
\hline \multicolumn{2}{|l|}{ VSD } & 2 & & \\
\hline \multicolumn{2}{|c|}{$V S D+T G A$} & 1 & & \\
\hline \multicolumn{2}{|c|}{ Pulmonalklappenstenose } & 1 & & \\
\hline \multicolumn{2}{|c|}{ Cor triatriatum } & 1 & & \\
\hline \multicolumn{5}{|c|}{ Tabelle 4 Assoziierte kardiale Fehlbildungen bei Patienten mit TAPVC } \\
\hline \multicolumn{5}{|c|}{ Hier eine Auflistung der Anomalien der 16 Patienten mit komplexen Herzfehlern: } \\
\hline Patient & Diagnosen & & & TAPVC Typ \\
\hline 1 & DORV, MGA, PS, & oplastischer LV, Hete & taxie & suprakardial \\
\hline 2 & VSD, hypoplastis & & & suprakardial \\
\hline 3 & AVSD, hypoplasti & r LV, Heterotaxie & & suprakardial \\
\hline 4 & DORV, MGA, PS, & D, Heterotaxie & & suprakardial \\
\hline 5 & DORV, AVSD, hyp & astischer LV & & suprakardial \\
\hline 6 & DORV, MGA, PS, & oplastischer LV, Hete & taxie & suprakardial \\
\hline 7 & Cor triatriatum & & & suprakardial \\
\hline 8 & DORV, MGA, PS, & oplastischer LV, Hete & taxie & gemischt \\
\hline 9 & PS & & & suprakardial \\
\hline 10 & VSD & & & kardial \\
\hline 11 & TGA, VSD, MI, PS & & & kardial \\
\hline 12 & AVSD, hypoplasti & RV, Heterotaxie & & infrakardial \\
\hline 13 & HLHS & & & kardial \\
\hline 14 & VSD, hypoplastis & LV, PS, TI & & kardial \\
\hline 15 & DORV, MGA, AVS & S, hypoplastischer L & Heterotaxie & fehlt \\
\hline 16 & VSD & & & fehlt \\
\hline
\end{tabular}

Tabelle 5 Auflistung aller assoziierten Fehlbildungen

Präoperativ bestehende pulmonale Hypertonie wurde bei insgesamt sieben $(3,6 \%)$ Patienten diagnostiziert. Neben der TAPVC, wiesen zwei dieser Patienten zusätzlich einen persistierenden Ductus Arteriosus auf zwei einen Ventrikelseptumdefekt und ein Patient Frühgeburtlichkeit. 


\subsubsection{Voroperationen}

Sieben Patienten $(3,6 \%)$ wurden bereits vor der eigentlichen Korrekturoperation am Herzen operiert. Bei 6 Patienten wurde vor der Korrektur eine Ballonatrioseptostomie nach Rashkind durchgeführt. Bei den übrigen Patienten war die Blutmischung durch ASDs bzw. PFOs auch ohne Intervention zur Genüge gewährleistet.

Die anschließende Korrekturoperation erfolgte 0, 2, 4 und 27 Tage nach diesem initialen Eingriff; In einem Fall allerdings erst vier Jahre später.

Bei einem Patient war vorher eine Ballonvalvuloplastie zur Behebung einer Pulmonalklappenstenose versucht worden, die allerdings erst im Rahmen der Korrekturoperation am Deutschen Herzzentrum endgültig korrigiert werden konnte.

\subsection{Korrekturoperation}

\subsubsection{Patientendaten}

Bei insgesamt 193 Patienten wurde eine TAPVC operativ korrigiert. Das Körpergewicht zum Zeitpunkt der Korrekturoperation lag im Durchschnitt bei 5,5 \pm 8,6 kg (Median=3,5kg); der leichteste Patient wog 1,8kg, der schwerste $64 \mathrm{~kg}$.

Die Körpergröße der Patienten lag zwischen $34 \mathrm{~cm}$ und $178 \mathrm{~cm}$ und betrug im Durchschnitt $58 \pm 21$ $\mathrm{cm}($ Median $=53 \mathrm{~cm})$.

Aus den Größen wurde für jeden Patienten der Körperoberfläche nach der Formel BSA $\left(\mathrm{m}^{2}\right)=$ 0.007184 x Größe $(\mathrm{cm})^{0.725} \times$ Gewicht $(\mathrm{kg})^{0.425}$ nach DuBois (Du Bois \& Du Bois, 1989) berechnet. Die durchschnittliche Körperoberfläche lag bei 0,28 $\pm 0,23 \mathrm{~m}^{2}$, mit einem Minimum von 0,17 $\mathrm{m}^{2}$ und einem Maximum von $1,77 \mathrm{~m}^{2}$.

\subsubsection{Alter bei Korrektur-OP}

Im Durchschnitt lag das Alter bei Operation bei $435 \pm 1981$ Tagen (Median $=21$ ). Dieser relativ hohe

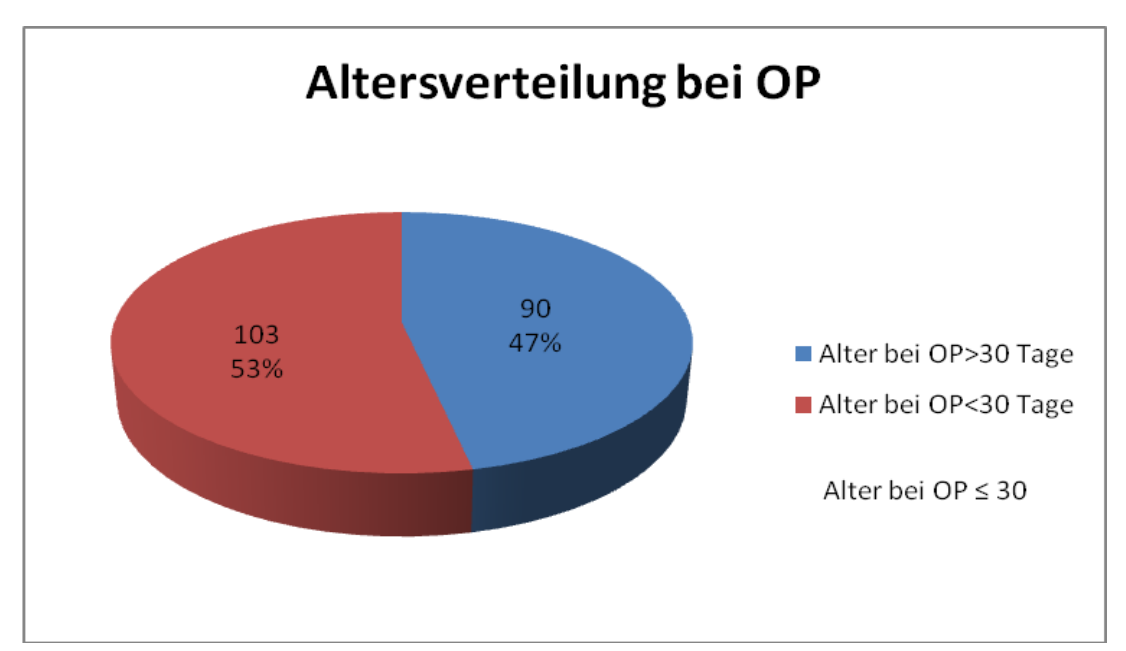
Mittelwert kommt allerdings, wie durch die Standardabweichung angedeutet, durch einzelne Patienten zustande, die erst im Erwachsenenalter operiert wurden. Denn wie Diagramm 3 zeigt, wurde bei 103 (53\%) Patienten die Korrekturoperation während der ersten 30 Lebenstagen - also in der Neugeborenenperiode - durchge- 
führt.

Diagramm 4 Altersverteilung zum Zeitpunkt der Korrektur-Operation

Die nächst größere Gruppe $(33,6 \%)$ ist die der Patienten, die zwischen 30. Lebenstag und vollendetem 6. Lebensmonat operiert wurden, gefolgt von den Patienten, die zwischen 7. Und 12. Monat operiert wurden operiert wurden

Fünf Patienten wurden vor dem nach dem ersten und vor dem sechsten Lebensjahr, zwei zwischen 6. Und

11.

Lebensjahr

operiert.

Diagramm 5 veranschaulicht diese Altersverteilung bei Operation deutlich und zeigt, dass Korrekturoperationen in fortgeschrittenem Alter nur vereinzelt vorkommen. Von den fünf Patienten, die im Erwachsenenalter operiert wurden, waren zwei 25 Jahre, zwei 30 Jahre und einer 51 Jahre alt.

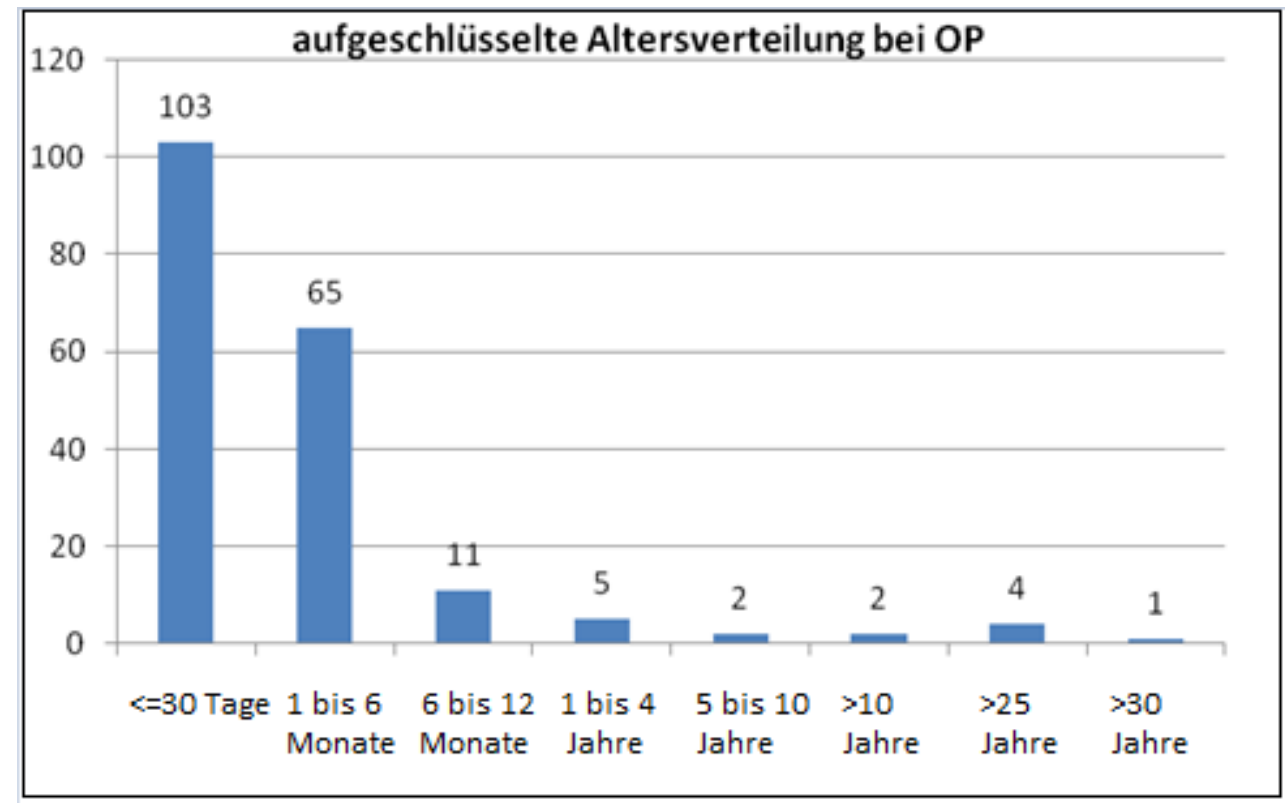

Diagramm 6 : Häufigkeitsverteilung des Patientenalters zum Zeitpunkt der Korrektur-Operation

\subsubsection{Durchgeführte Prozeduren}

Insgesamt wurde bei 193 Patienten die Lungenvenenfehlmündung operativ korrigiert. 181 Operationen waren isolierte TAPVC-Korrekturen. Bei 15 Operationen fanden wegen kardialer Begleitfehlbildungen zusätzlich noch weitere Prozeduren statt. Bei 8 Prozeduren war dies der erste Schritt zur Palliation eines univentrikulären Herzens war.

\begin{tabular}{ll}
\hline $\begin{array}{l}\text { Zusätzliche Prozedur } \\
\text { während Korrektur-OP }\end{array}$ & Anzahl \\
\hline aortopulmonaler Shunt & 5 \\
Norwood - Operation & 2 \\
modifizierter Blalock-Taussig-Shunt & 1 \\
VSD-Verschluss & 4 \\
Rastelli-Operation & 1 \\
Pulmonalklappenplastik & 1 \\
\hline
\end{tabular}


Cor Triatriatum Korrektur 1

Tabelle 6 Liste der im Rahmen der Korrektur-Operation zusätzlich durchgeführter chirurgischer Maßnahmen

\subsubsection{OP-Zeiten}

Die Operation erfolgte bei allen Patienten mit Hilfe der Herz-Lungen-Maschine bei extrakorporaler Zirkulation und in Hypothermie.

Die Korrekturoperation dauerte durchschnittlich $204 \pm 64$ min (Median = 193min), dabei dauerte die schnellste OP $70 \mathrm{~min}$ und die längste $450 \mathrm{~min}$.

Die Dauer der extrakorporalen Zirkulation betrug im Mittel $62 \pm 36$ min (Median = 51min). Die längste Bypasszeit war 246 min, die kürzeste 18 min lang. Dabei sollte erwähnt werden, dass bei dem Patienten mit der längsten Bypasszeit zusätzlich zur Korrektur der TAPVC eine Norwood-OP durchgeführt wurde.

Die durchschnittliche Aortenklemmzeit lag bei $47 \pm 17$ min (Median = 47min), wobei die längste 145 min und die kürzeste 7 min betrug. Auch hier war die OP mit der längsten Abklemmzeit der Aorta keine alleinige TAPVC-Korrektur, sondern mit einer Rastelli-OP kombiniert.

Bei 75,6\% der Patienten wurde die Anastomose im totalen Kreislaufstillstand und tiefer Hypothermie (circa 18 Grad) durchgeführt, bei 23,5\% erfolgte eine kontinierliche Perfusion in moderater Hypothermie ( circa 28 Grad). Dabei ist in der letzten Dekade ein Trend weg von der Operationstechnik bei totalem Kreislaufstillstand zu beobachten. Vor dem Jahr 2003 wurden n=5, 3\% der Operationen bei kontinuierlicher extrakorporaler Zirkulation durchgeführt , wohingegen seit 2003 76\% der Operationen bei kontinuierlicher extrakorporaler Zirkulation durchgeführt wurden. Bei den Operationen, bei denen ein totaler Kreislaufstillstand angewendet wurde, betrug die Zeit des Kreislaufstillstands durchschnittlich $44 \pm 15$ min (Median = 46min), der kürzeste Kreislaufstillstand war 7 min und der längste 98 min lang.

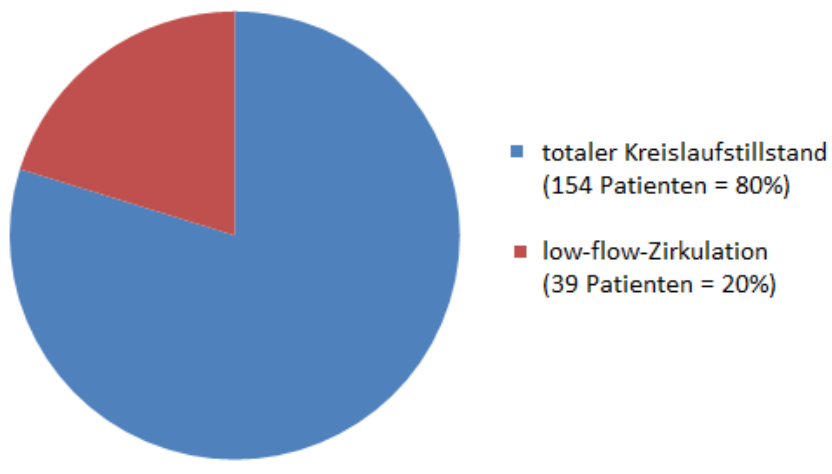

Diagramm 7 Anzahl der Korrektur-OPs bei totalem Kreislaufstillstand und bei Low-flow-Zirkulation

Die tiefste Körpertemperatur während der Operation lag zwischen $15^{\circ} \mathrm{C}$ und $36^{\circ} \mathrm{C}$, im Durchschnitt bei $21 \pm 5{ }^{\circ} \mathrm{C}$ (Median $=19^{\circ} \mathrm{C}$ ). Der niedrige Median erklärt sich durch die Tatsache, dass bei $80 \%$ der Patienten die Operation bei totalem Kreislaufstillstand durchgeführt wurde, wozu eine tiefe Hypothermie erreicht werden muss. Bei Operationen unter Low-flow-Zirkulation liegt die Temperatur zwischen 24 und $32{ }^{\circ} \mathrm{C}$ (,moderate Hypothermie“). 


\subsubsection{Nahtmaterial}

Als Nahtmaterial für die Anastomosierung der Lungenvenen wurden entweder resorbierbare oder nicht resorbierbare Nähte verwendet. Resorbierbares Nahtmaterial wurde in $75 \%$ der Operationen verwendet. Bei den übrigen $25 \%$ wurden nicht-resorbierbare Nähte verwendet. Von den nicht resorbierbaren Nähten waren $82 \%$ Polypropylenfäden (Prolene®), $10 \%$ beschichtete Polyesterfäden (Ethibond®) und $8 \%$ andere nicht resorbierbare Nähte (z.B. Goretex®). Als resorbierbares Nahtmaterial wurden zu 98\% Polydioxanonfäden (PDS) verwendet, die restlichen $2 \%$ waren Polygalaktinfäden (Vicryl@).

In diesem Kollektiv ist ein zeitlicher ein Wendepunkt bei der Verwendung unterschiedlichen Nahtmaterials zu verzeichnen. Nach 1983 wurden ausschließlich resorbierbare Nähte zur Herstellung der Lungenvenenanastomose verwendet.

\subsubsection{Stationärer Aufenthalt}

Der stationäre Aufenthalt nach der Korrekturoperation betrug im Schnitt $20 \pm 18$ Tage (Median $=17$ Tage). Die kürzeste Aufenthaltsdauer war 0 Tage (Exitus in tabula) und der längste Aufenthalt war 153 Tage lang.

Während des stationären Aufenthaltes war bei keinem Patienten eine Re-OP nötig. Lediglich bei acht Patienten erfolgte der Thoraxverschluss sekundär auf Intensivtation, was allerdings nicht als ReOperation gewertet wurde.

Patienten wurden nach der OP intubiert auf die Intensivstation verlegt. Die postoperative Beatmungsdauer betrug zwischen 1 und 40 Tagen. Die durchschnittliche Dauer der Intubation betrug $5 \pm$ 6 Tage (Median $=4$ Tage).

\subsubsection{Operative Letalität}

Die Letalität während der Korrekturoperation betrug im gesamten Patientengut 1,5\% - drei Patienten sind während der Operation verstorben. Alle drei Operationen waren isolierte Korrekturoperation einer TAPVC, zusätzliche Herzfehler bestanden keine. Bei einem Patienten bestand präoperativ eine pulmonale Obstruktion, keiner wurde notfallmäßig operiert. Die Todesursachen sind in der folgenden Tabelle aufgeführt.

\begin{tabular}{llllllll}
\hline Patient & Pin & Typ & PVo & $\begin{array}{l}\text { OP-Alter } \\
\text { (in Tagen) }\end{array}$ & $\begin{array}{l}\text { Min. } \\
\text { Temp }\end{array}$ & Naht & Todesursache \\
\hline 1 & 9272 & Supra & nein & 14 & $17^{\circ} \mathrm{C}$ & resorb. & Myokardversagen \\
\hline 2 & 30022 & Supra & nein & 3654 & $15^{\circ} \mathrm{C}$ & nicht r. & $\begin{array}{l}\text { Hirnödem, Myokardvera- } \\
\text { gen (Ventrikel mit Einzel- } \\
\text { nekrosen) }\end{array}$ \\
\hline 3 & 6151 & infra & ja & 4 & $21^{\circ} \mathrm{C}$ & nicht r. & Myokardversagen \\
\hline
\end{tabular}

Tabelle 7 intraoperative Letalität der Korrektur-OP 


\subsubsection{Frühletalität}

Die Frühletalität, hier definiert als Krankenhausletalität nach der Korrekturoperation, betrug 7,8\% (15 von 193 Patienten). Drei davon starben, wie oben erwähnt, während der Korrekturoperation (in der Tabelle blau unterlegt).

\begin{tabular}{|c|c|c|c|}
\hline $\mathrm{PIZ}$ & Diagnose & $\begin{array}{l}\text { Tage zwischen } \\
\text { OP und Tod }\end{array}$ & Todesursache \\
\hline 9272 & TAPVC supra & 0 & low-cardiac-output \\
\hline 6151 & $\begin{array}{l}\text { TAPVC infra, präop. Ob- } \\
\text { struktion }\end{array}$ & 0 & low-cardiac-output \\
\hline 30022 & TAPVC supra & 0 & $\begin{array}{l}\text { Hirnödem } \\
\text { low-cardiac-output }\end{array}$ \\
\hline 24000 & $\begin{array}{l}\text { TAPVC, SV, Pulmonalste- } \\
\text { nose }\end{array}$ & 38 & unbekannt \\
\hline 42623 & $\begin{array}{l}\text { TAPVC supra, SV, Hete- } \\
\text { rotaxie }\end{array}$ & 10 & $\begin{array}{l}\text { Sepsis nach sek. Thoraxverschluß } \\
\text { low-cardiac-output } \\
\text { Kammerflimmern }\end{array}$ \\
\hline 23523 & $\begin{array}{l}\text { TAPVC supra, SV, präop. } \\
\text { Obstruktion }\end{array}$ & 1 & unbekannt \\
\hline 17557 & $\begin{array}{l}\text { TAPVC supra, SV, Hete- } \\
\text { rotaxie, präop. Obstrukti- } \\
\text { on }\end{array}$ & 1 & low-cardiac-output \\
\hline 41262 & $\begin{array}{l}\text { TAPVC kardial, SV (HLH), } \\
\text { CoA }\end{array}$ & 4 & $\begin{array}{l}\text { Rechtsherzversagen bei Lungen- } \\
\text { embolie }\end{array}$ \\
\hline 11171 & TAPVC infra & 1 & $\begin{array}{l}\text { low-cardiac-output } \\
\text { hypox. Hirnschaden }\end{array}$ \\
\hline 3684 & TAPVC supra & 22 & Sepsis \\
\hline 46306 & TAPVC supra & 11 & Pneumonie \\
\hline 21870 & $\begin{array}{l}\text { TAPVC infra, präop. Ob- } \\
\text { struktion, pulm. Hyperto- } \\
\text { nie }\end{array}$ & 92 & $\begin{array}{l}\text { Lungenvenenstenose, pulmonale } \\
\text { Hypertonie. Pneumonie }\end{array}$ \\
\hline 46067 & $\begin{array}{l}\text { TAPVC supra, präop. Ob- } \\
\text { struktion }\end{array}$ & 8 & Sepsis \\
\hline 46937 & $\begin{array}{l}\text { TAPVC supra, präop. Ob- } \\
\text { struktion }\end{array}$ & 1 & Lungenstauung, Hirnödem \\
\hline 15322 & TAPVC kardial & 29 & Rechtsherzversagen \\
\hline 45733 & $\begin{array}{l}\text { TAPVC supra, präop. Ob- } \\
\text { struktion }\end{array}$ & 2 & $\begin{array}{l}\text { besser Linksherzversagen, Nie- } \\
\text { renversagen }\end{array}$ \\
\hline 21072 & $\begin{array}{l}\text { TAPVC supra, präop. Ob- } \\
\text { struktion }\end{array}$ & 3 & $\begin{array}{l}\text { Rechtsherzversagen, Lungenstau- } \\
\text { ung }\end{array}$ \\
\hline 49348 & $\begin{array}{l}\text { TAPVC infra, präop. Ob- } \\
\text { struktion }\end{array}$ & 1 & Rhythmusstörungen \\
\hline
\end{tabular}

Tabelle 8 Liste aller frühen Todesfälle mit Todesursachen 


\subsection{Follow - up}

Für das Follow-up konnten Daten von insgesamt 95,3\% der operierten Patienten erhoben werden. Die Follow-up Zeit (= Zeit zwischen Korrekturoperation und dieser Erhebung zugrunde liegenden Datenerhebung im Jahre 2012) lag im Mittel bei $15 \pm 11$ Jahren, dabei betrug die längste Nachbeobachtungszeit 38 Jahre.

Neun Patienten (4,7\%) ließen sich im Jahre 2012 nicht kontaktieren, auch wenn es für diese noch für 7,3 \pm 8 ,8 Jahre nach der Operation Aktennotizen und Arztbriefe gab. Fünf von diesen Patienten sind entweder gleich nach der Operation oder später ins Ausland verzogen und waren selbst durch die ausländischen Meldeämter nicht ausfindig zu machen. Der Verbleib der anderen vier konnte nicht festgestellt werden.

Aus der Gesamtheit dieser Daten ergibt sich eine kumulative Follow-up Zeit von 2902 Patientenjahren.

\subsubsection{Re-Operationen}

\subsubsection{Anzahl der Re-Operationen in der gesamten Kohorte}

Von den insgesamt 193 Patienten mussten 30 (15,5\%) Patienten einer weiteren Operation am Herzen unterzogen werden. Davon waren 14 (7\%) Re-Operationen wegen Stenosierung der Lungenvenen und 16 Re-OPs aufgrund anderer kardialer Probleme.

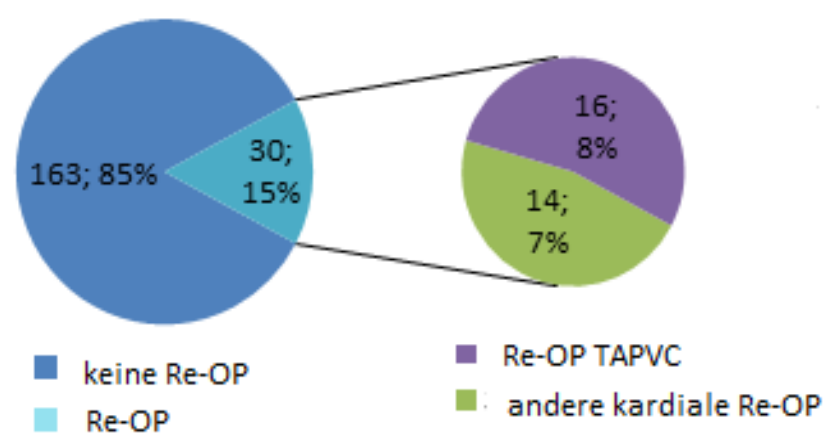

Tabelle 9 Anteil an Re-Operationen in der gesamten Kohorte

Daraus ergibt sich folgende, die Freiheit von Re-Operationen nach Korrekturoperation beschreibende, Kaplan-Meier-Kurve.

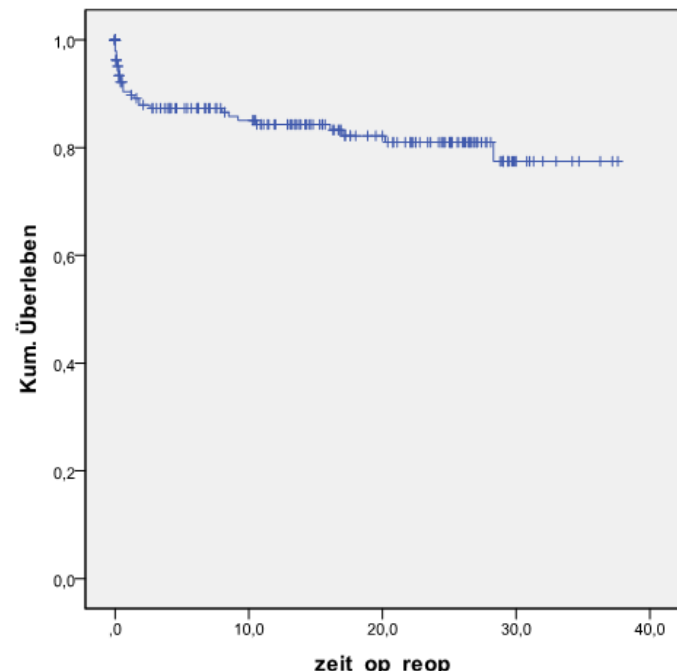

1Wahrscheinlichkeitsdarstellung für Freiheit von Re-OPs nach Kaplan Meier 


\section{$\underline{\text { Re-OPs bei Patienten mit isolierter TAPVC (177 Patienten) }}$}

Betrachtet man das Kollektiv der Patienten mit isolierter TAPVC gesondert, ergibt sich ein Kollektiv von 177 Patienten. Bei 22 Patienten mit isolierter TAPVC erfolgte eine Re-OP, das sind 12,4\%. Davon musste bei 12 Patienten (7\%) eine Stenose der Pulmonalvenen erweitert werden, bei 6 Patienten erfolgten andere kardiale Re-Operationen (ein ASD-Verschluss, ein VSD-Verschluss, eine Ligatur des PDA und 2 andere Operationen).

\section{$\underline{\text { Re-OPs bei Patienten mit komplexen Vitien (16 Patienten) }}$}

Von den 16 Patienten mit komplexen Herzvitien wurden nach TAPVC-Korrektur bei 8 Patienten (50\%) eine oder mehrere Re-Operationen durchgeführt: 3 partielle cavopulmonale Anastosen (einer anschließend totale cavopulmonale Anastose), 1 Klappenplastik, 2 modifizierte Blalock-Taussig-Shunts (davon bei einem zusätzlich Korrektur einer PVO), 2 Korrekturen einer PVO (davon einer zusätzlich mBT-Shunt).

\subsubsection{Ursachen für Re-Operationen}

1. Pulmonalvenenstenose

Der Grund für eine Re-Korrektur der initial hergestellten Anastomose war immer eine Stenosierung der Anastomose, nie eine Insuffizienz. Dabei musste die initial hergestellte Anastomose zwischen Pulmonalvenen und Vorhof erweitert werden. Von 14 Patienten, die wegen einer Pulmonalvenenstenose reoperiert wurde, wiesen lediglich 6 Patienten bereits präoperativ eine pulmonalvenöse Obstruktion auf.

\section{Art der Pulmonalvenenstenosenkorrektur}

Bei 8 der 14 Patienten wurde die Stenose mit einem Patch, bei 5 wurde die Stenose reseziert und eine erneute Anastomose durchgeführt und bei einem Patienten wurde eine interventionelle Ballondilatation durchgeführt.

Bei einem Patienten war die Pulmonalvenenstenose nach der Reoperation rekurrent und wurde zwei Mal interventionell dilatiert. Eine mäßiggradige Stenose blieb bestehen. Der Patient verstarb an einer massiven gastrointestinalen Blutung.

\section{Zeit zwischen initialer OP und Re-OP}

Von den 14 Re-Stenosen wurden 8 innerhalb des ersten Jahres nach Korrektur reoperiert, davon wurde ein Patient noch im Rahmen des ersten Aufenthaltes reoperiert. 2 Patienten 
wurden innerhalb der ersten 2 Jahre, einer nach 3 Jahren, einer nach 8 Jahren, einer nach 16 und einer nach 17 Jahren reoperiert.

2. Andere kardiale Re-Operationen

Für kardiale Re-Operationen anderer Genese gab es unterschiedliche Gründe.

a. ASD-Verschluss

Dies war die häufigste Ursache für kardiale Re-Operationen. Bei einigen Patienten wurde der ASD aus hämodynamischen Gründen initial offen belassen und anschließend elektiv verschlossen.

b. Palliation univentrikulärer Malformationen

Nachdem das Patientenkollektiv auch einige Patienten mit univentrikulärem Herzen inkludiert, mussten zur definiven Palliation dieser Malformationen mehrere typische operative Schritte (Shunt-Anlage, PCPC, TCPC) durchgeführt werden. Gemeinsame Endstrecke dieser Operationen ist die Herstellung einer Zirkulation nach Fontan. Von insgesamt 11 Patienten mit einem univentrikulären Herzen wurden 7 Patienten reoperiert. Bei zwei Patienten wurde nach TAPVC-Korrektur ein Shunt zwischen System- und Lungenkreislauf angelegt (bei allen anderen erfolgte dieser Schritt im Rahmen der TAPVC-Korrektur). Bei drei Patienten konnte eine anschließende PCPC durchgeführt werden (s.o.). Bei einem dieser drei Patienten konnte eine totale cavopulmonale Anastomose hergestellt werden (Fontan-Zirkulation). Vier Patienten mit univentrikulärem Herzen verstarben, bevor palliative Schritte vorgenommen werden konnten. Drei davon direkt im Anschluss an die TAPVC-Korrektur, ein Patient nach knapp drei Jahren, ohne dass eine Re-Operation durchgeführt wurde (DORV, Pulmonalarterienstenose, hochgradige AV-Klappeninsuffizient, Z.n. zerebralem Krampfgeschehen, Z.n. intracerebraler Blutung, ausgeprägter hypoxischer Hirnschaden).

c. Klappenrekonstruktionen

Bei insgesamt zwei Patienten wurden Klappenrekonstruktionen bei Mitralklappeninsuffizienz bzw. Trikuspidalklappenanomalie durchgeführt.

Zusammenfassend hier eine Liste aller durchgeführten Re-Operationen

\begin{tabular}{lc} 
Durchgeführte Operation & Anzahl \\
\hline Re-OP TAPVC (=PVO-Korrektur) & $\mathbf{1 4}$ \\
\hline Andere kardiale Re-OPs: & $\mathbf{1 6}$ \\
ASD-Verschluss & 7 \\
(davon 1 ASD- und VSD-Verschluss) & \\
PCPC & 3 \\
(davon anschl. 1 TCPC) & $(1)$ \\
Klappen-OP & 2 \\
\hline
\end{tabular}




\begin{tabular}{ll}
\hline PDA-Verschluss & 1 \\
mod. BT-Shunt & 2 \\
\hline
\end{tabular}

Tabelle 10 Liste aller Re-Operationen

\subsubsection{Letalität der Re-operierten Patienten}

Neun der insgesamt 30 Re-Operierten (30\%) waren zum Zeitpunkt der Datenerhebung verstorben; davon 2 unmittelbar postoperativ, womit sich eine Frühletalität von 6,7\% ergibt.

\section{1) Differenzierung der Letalitätsraten je nach Patientengruppen}

a) Patienten mit komplexen Vitien (16 Patienten)

Von den neun nach Re-Operation verstorbenen Patienten hatten sechs eine zusätzliche kardiale Anomalie und zählten somit zur Gruppe der komplexen Anomalien. 8 Patienten mit komplexen Vitien wurden reoperiert, 6 davon starben. Das ergibt eine Letalität für die Gruppe der reoperierten Patienten mit komplexen Anomalien von 75\%. Die zwei, die zum Ende der Datenerhebung noch lebten, hatten univentrikuläre Malformationen und befanden sich im Stadium II der Fontan-Korrektur (PCPC).

b) Patienten mit isolierter TAPVC (177 Patienten)

Von den 22 reoperierten Patienten der 177 Patienten mit isolierter TAPVC starben drei Patienten. Das ergibt für die Gruppe der reoperierten Patienten mit initial isolierter TAPVC eine Letalität von $13,6 \%$.

\section{2) Differenzierung der Letalitätsraten je nach Art der Re-OP}

Letalität nach Re-OPs bei Pulmonalvenenstenose

Wenn man die Gruppe der Patienten mit postoperativer Pulmonalvenenstenose separat betrachtet, errechnet sich eine Letalität von 28,6\% nach der Re-Operation (4 von 14 Patienten sind verstorben). Von diesen vier verstorbenen Patienten starben zwei an nicht kardial bedingten Ursachen, bei den anderen zwei Patienten ist die Todesursache nicht bekannt. Allerdings war bei einem dieser Patienten in einer Herzkatheteruntersuchung vier Monate vor dem Ableben bereits eine signifikante pulmonalvenöse Obstruktion aufgefallen, die allerdings keine Therapieoptionen offen ließ. Zusätzlich bestanden weitere nicht kardiale Diagnosen, die zur infausten Prognose beitrugen.

\section{Letalität nach anderen kardiale Operationen}

Die Patientengruppe mit univentrikulären Malformationen hat eine sehr schlechte Prognose. Von den insgesamt sieben reoperierten Patienten mit univentrikulärem Herzen verstarben fünf. Die anderen zwei Patienten lebten bei Abschluss der Datenerhebung, einer ( 3 Jahre alt) mit kompletter Fontan-Zirulation, einer (2Jahre alt) mit partieller cavopulmonaler Anastomose. Wie bereits oben erwähnt starben vier Patienten, bevor operative Schritte zur Palliation vorgenommen werden konnten.

Zusammenfassend hier eine Übersicht der Re-Operationen und Letalitätsraten: 


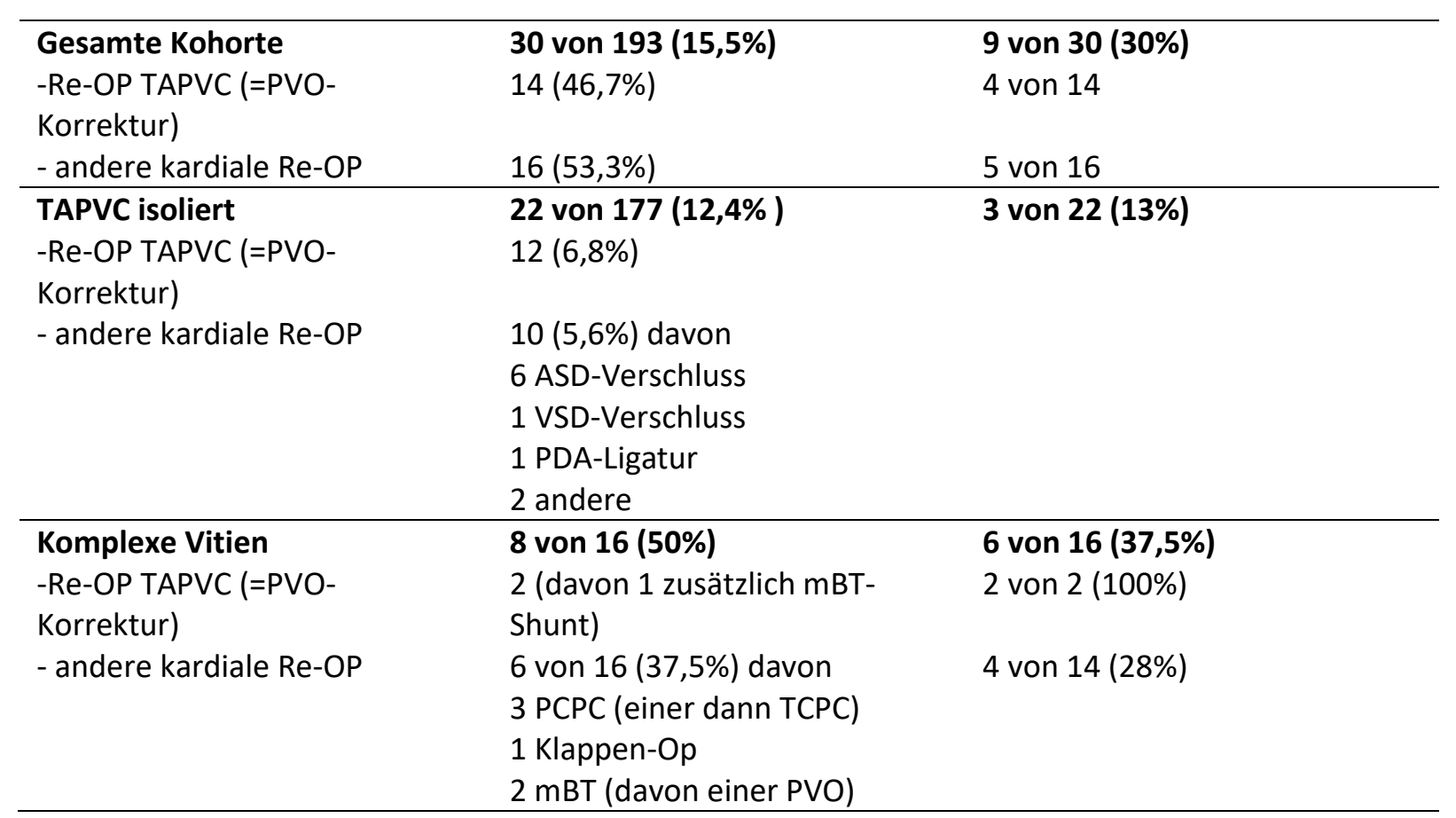

Tabelle 11 Liste aller Re-Operationen und Letalitätsraten

\subsubsection{Gesamtletalität}

Von den 193 korrigierend operierten Patienten waren zum Zeitpunkt der Datenerhebung 31 verstorben (16,1\%). Davon verstarben 18 Patienten (58,1\% der Verstorbenen, 9,3\% aller Operierten) während des Krankenhausaufenthaltes zur Korrektur-OP. Die übrigen 13 Patienten verstarben nach ihrer Entlassung.

Von den 31 verstorbenen Patienten hatten 21 eine isolierte TAPVC und 10 Patienten komplexe Anomalien. Nahezu ein Drittel aller verstorbenen Patienten gehört zur Untergruppe der Patienten mit komplexen Herzfehlern.

Somit ergibt sich für die Untergruppe der isolierten TAPVC mit 21 verstorbenen Patienten von insgesamt 177 Patienten eine Letalität von 11,9\%. Von den anderen 16 Patienten mit komplexeren Vitien starben 10 Patienten. Damit ergibt sich eine Letalität von $62 \%$. Diese Aufschlüsselung zeigt, dass die insgesamte Letalität von $16,1 \%$ vor allem durch die hohe Letalitätsrate der Patienten mit assoziierten Herzfehlern zustande kommt. Die Letalität derer mit isolierter TAPVC ist deutlich geringer. 


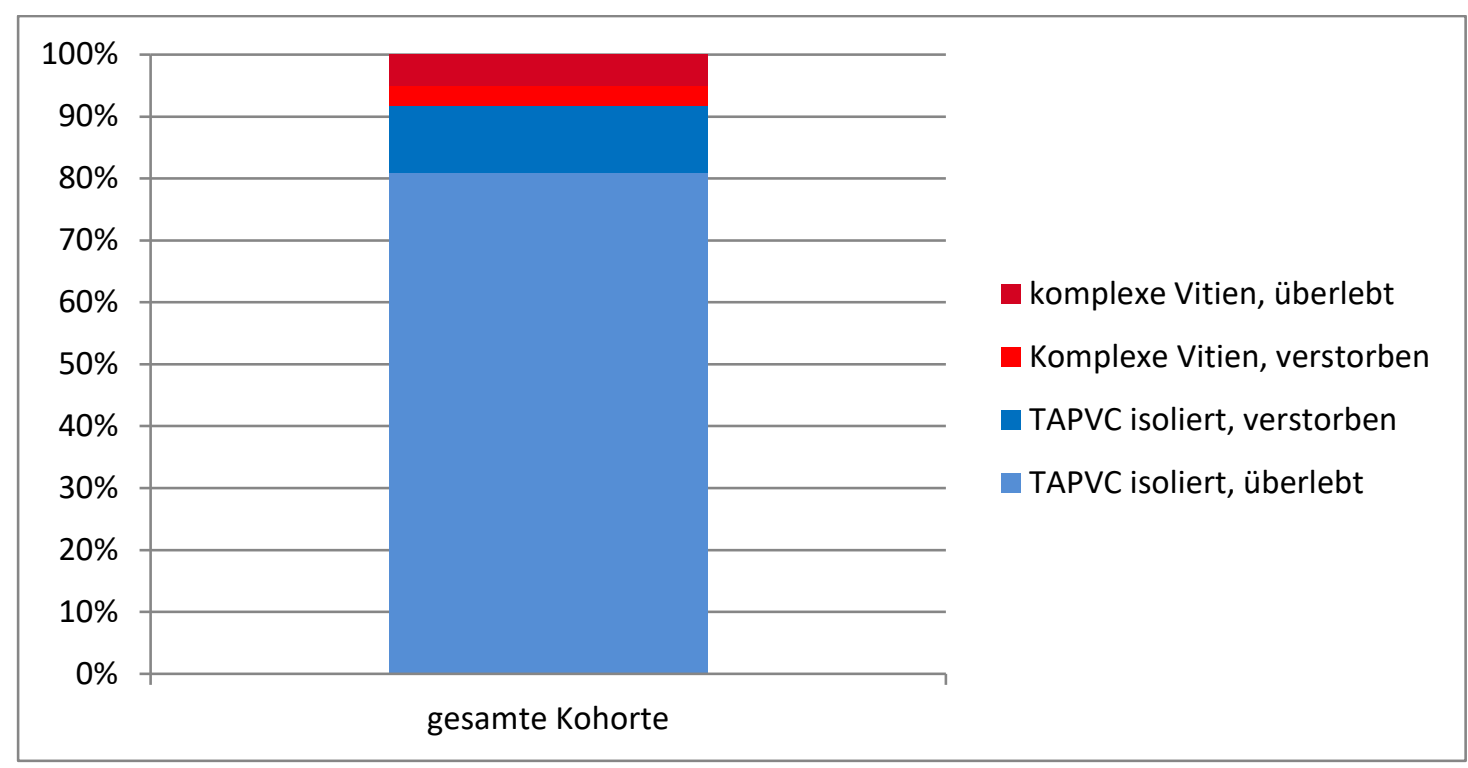

Diagramm 8 Anteil der lebenden und verstorbenen Patienten, geteilt nach Untergruppen TAPVC isoliert und komlexe Vitien

\subsubsection{Frühmletalität}

Insgesamt starben 18 Patienten (9\% des gesamten Patientenkollektives) im Rahmen des ersten stationären Aufenthaltes (s.o.). Das sind 58,1\% aller bislang Verstorbenen, womit der Frühletalität ein höherer Anteil als der folgenden Spätletalität zufällt

\subsubsection{Spätletalität (Patienten, die nach Entlassung verstarben)}

Die übrigen 13 bislang verstorbenen Patienten verstarben zu späteren Zeitpunkten. Das ergibt eine Spätletalität von 6,74\%. Von den meisten (neun Patienten) ist die Todesursache nicht bekannt. Hier konnten trotz intensiven Nachforschens nur Verdachtsdiagnosen aufgestellt werden (unbehandelte

\begin{tabular}{|l|l|l|l|} 
PIZ & OP-Zeitpunkt & Todeszeitpunkt & Erreichtes Alter \\
\hline $\mathbf{1 0 3 8}$ & 21.05 .1990 & 07.08 .1990 & 2,6 Monate \\
\hline $\mathbf{4 5 7 7}$ & 23.09 .1991 & 11.12 .1991 & 2,6 Monate \\
\hline $\mathbf{1 5 3 5 1}$ & 25.10 .1983 & 15.03 .1984 & 4,7 Monate \\
\hline $\mathbf{5 4 8 9}$ & 28.08 .1992 & 15.03 .1993 & 6,6 Monate \\
\hline $\mathbf{4 7 5 7 9}$ & 12.08 .2009 & 13.02 .2011 & 1,5 Jahre \\
\hline $\mathbf{1 5 3 0 2}$ & 06.02 .1983 & 27.07 .1984 & 1,5 Jahre \\
\hline $\mathbf{1 5 3 3 7}$ & 18.03 .1980 & 23.10 .1981 & 1,6 Jahre \\
\hline $\mathbf{1 3 4 1 7}$ & 21.04 .1997 & 11.06 .1999 & 2,1 Jahre \\
\hline $\mathbf{3 0 9 9 2}$ & 17.05 .2002 & 19.03 .2005 & 2,8 Jahre \\
\hline $\mathbf{1 0 6 6 4}$ & 18.05 .1995 & 05.10 .2011 & 6,4 Jahre \\
\hline $\mathbf{3 0 5 1 3}$ & 26.02 .2002 & 10.12 .2010 & 8,8 Jahre \\
\hline $\mathbf{4 9 3 4 5}$ & 20.05 .1981 & 30.09 .1991 & 10,4 Jahre \\
\hline $\mathbf{1 5 3 4 9}$ & 05.12 .1988 & 28.10 .2007 & 18,9 Jahre \\
\hline
\end{tabular}

Pneumonie, plötzlicher Herztod, etc). Von den wenigen Patienten, von denen die Todesursache bekannt ist verstarb keiner an kardialen Ursachen (Enteritis, fulminante GIBlutung, cerebrale Schädigung).

Die meisten Patienten verstarben in den ersten 5 Lebensjahren, 11 von 13 Patienten erreichten das 10. Lebensjahr nicht. 


\section{4 Überlebenswahrscheinlichkeit}

\subsubsection{Gesamte Kohorte}

Die Überlebenskurve nach Kaplan Meier aller Patienten ergab eine Überlebenswahrscheinlichkeit von $85,2 \pm 2,5 \%$ nach 5 Jahren, $84,5 \pm 2,7 \%$ nach 10 Jahren und $82.7 \pm 2.9 \%$ nach 20 und nach 30 Jahren.

\begin{tabular}{lllll}
\hline Überlebenswahrscheinlichkeit & nach 5 Jahre & nach 10 Jahren & nach 20 Jahren & nach 30 Jahren \\
\hline Gesamtes Kollektiv & $85,2 \pm 2,5 \%$ & $84,5 \pm 2,7 \%$ & $82,7 \pm 2,9 \%$ & $82,7 \pm 2,9 \%$ \\
TAPVC isoliert & $89,1 \pm 2,4 \%$ & $89,1 \pm 2,4 \%$ & $87,2 \pm 2,7 \%$ & $87,2 \pm 2,7 \%$ \\
Komplexer Herzfehler & $49,2 \pm 12,7 \%$ & $24,6 \pm 13,8 \%$ & & \\
\hline
\end{tabular}

Tabelle 13 Überblick der Überlebenswahrscheinlichkeiten

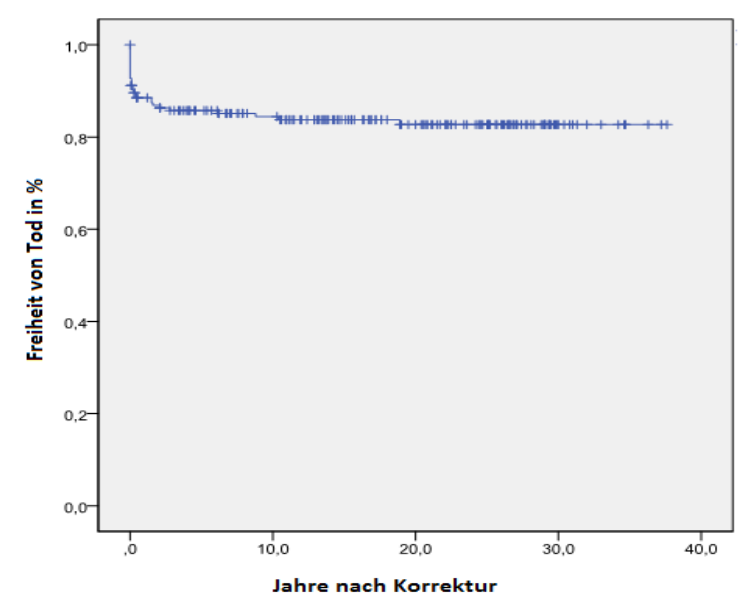

2 Überlebenskurve der ges. Kohorte nach Kaplan Meier

Eine Unterteilung der Patienten in zwei Gruppen, die mit isolierter TAPVC und die mit komplexeren Herzvitien, ergibt folgende Überlebenswahrscheinlichkeiten:

\begin{tabular}{|c|c|c|c|c|}
\hline TAPVC isoliert vs komplex & Anzahl & $\begin{array}{l}\text { Überlebenswahrschein- } \\
\text { lichkeit nach } 5 \text { Jahren }\end{array}$ & Nach 10 Jahren & $\begin{array}{l}\text { Nach } 20 \\
\text { Jahren }\end{array}$ \\
\hline TAPVC isoliert & 177 & $89,1 \pm 2,4 \%$ & $89,1 \pm 2,4 \%$ & $87,2 \pm 2,7 \%$ \\
\hline $\begin{array}{l}\text { davon mit pulmonaler Hyperto- } \\
\text { nie }\end{array}$ & 7 & $85,7 \pm 13,2 \%$ & & \\
\hline Komplexer Herzfehler & 16 & $49,2 \pm 12,7 \%$ & $24,6 \pm 13,8 \%$ & \\
\hline SV & 11 & $24,2 \pm 13,8 \%$ & $0 \%$ & $0 \%$ \\
\hline Heterotaxie & 7 & $38,1 \pm 19,9 \%$ & $0 \%$ & $0 \%$ \\
\hline andere & 2 & & & \\
\hline
\end{tabular}

Tabelle 14 Übersicht Überlebenswahrscheinlichkeiten der verschiedenen Untergruppen 


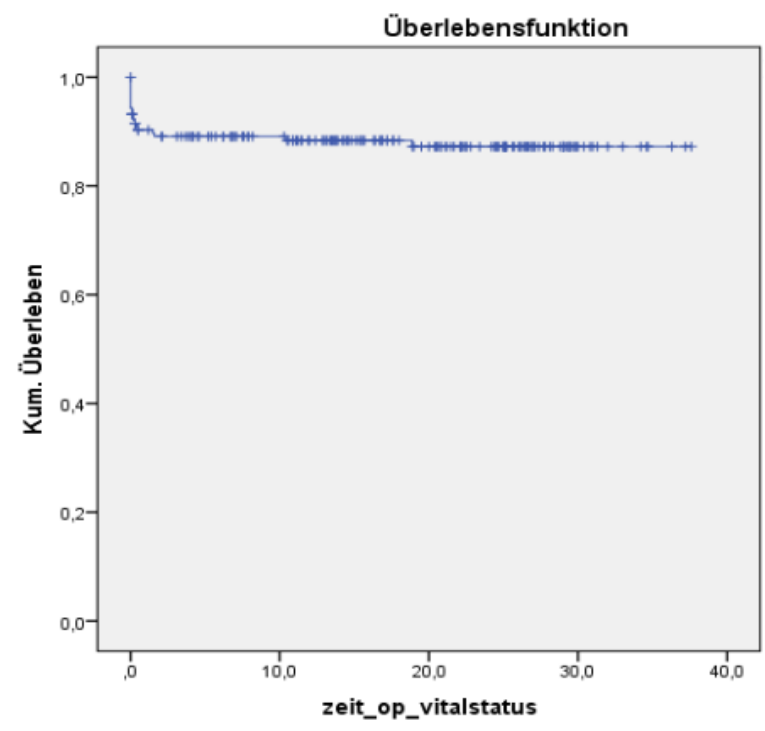

\subsubsection{Patienten mit isolierter TAPVC}

Die Überlebenswahrscheinlichkeit für Patienten mit isolierter TAPVC ist, verglichen mit den anderen Untergruppen, am höchsten und bleibt über die Jahre nahezu konstant. Nach fünf Jahren beträgt sie $89,1 \pm$ 2,4 \%, ebenso nach 10 Jahren. Nach 20 Jahren beträgt die Lebenswahrscheinlichkeit mit 87,2 $\pm 2,7 \%$ geringfügig weniger. Patienten mit isolierter TAPVC zeigen somit auch langfristig eine hohe Überlebenswahrscheinlichkeit nach erfolgreicher Korrektur.

3 Überlebenskurve TAPVC isoliert nach Kaplan Meier

\subsubsection{Patienten mit präoperativ bestehender pulmonaler Hypertonie}

Von den sieben Patienten mit präoperativ dokumentierter pulmonaler Hypertonie ist bis zum Zeitpunkt der Datenerhebung nur einer verstorben, womit es in der statistischen Auswertung nur ein Ereignis gab, was die Überlebensanalyse nach Kaplan-Meier/log rank Test in ihrer Voraussagekraft einschränkt. Die Überlebenswahrscheinlichkeit nach 5 Jahren beträgt $85,7 \pm 13,2 \%$.

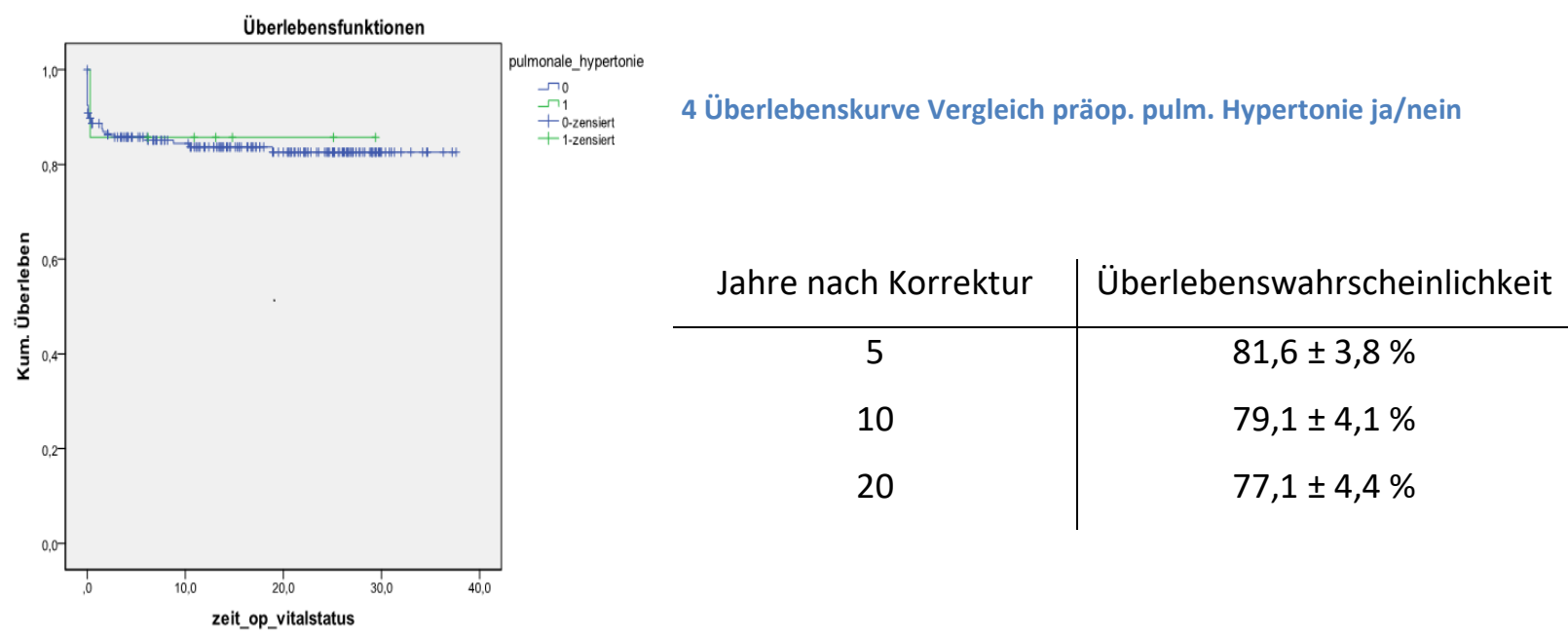

\subsubsection{Patienten mit zusätzlichen Anomalien des Herzens (komplexe Herzfehler)}

Diese Untergruppe an Patienten hat eine sehr niedrige Überlebenswahrscheinlichkeit. Die zwei häufigsten zusätzlichen Malformationen (die mit univentrikulären Malformationen und darunter diese mit Heterotaxie) werden unten gesondert angeführt.

Die Überlebenswahrscheinlichkeit eines Patienten mit TAPVC und zusätzlicher kardialer Anomalie beträgt nach einem Jahr $68,8 \pm 11,6 \%$. Bereits nach fünf Jahren nach der Korrektur beträgt sie nur noch $49,2 \pm 12,7 \%$ und nach 10 Jahren nur noch $24,6 \pm 13,8 \%$. Dazu ist allerdings zu sagen, dass die Patienten die zum Zeitpunkt der Datenauswertung noch lebten, noch nicht alle das 10. postoperative Jahr erreicht hatten. 
Die Kaplan-Meier-Kurve für diese Gruppe zeigt Folgendes:

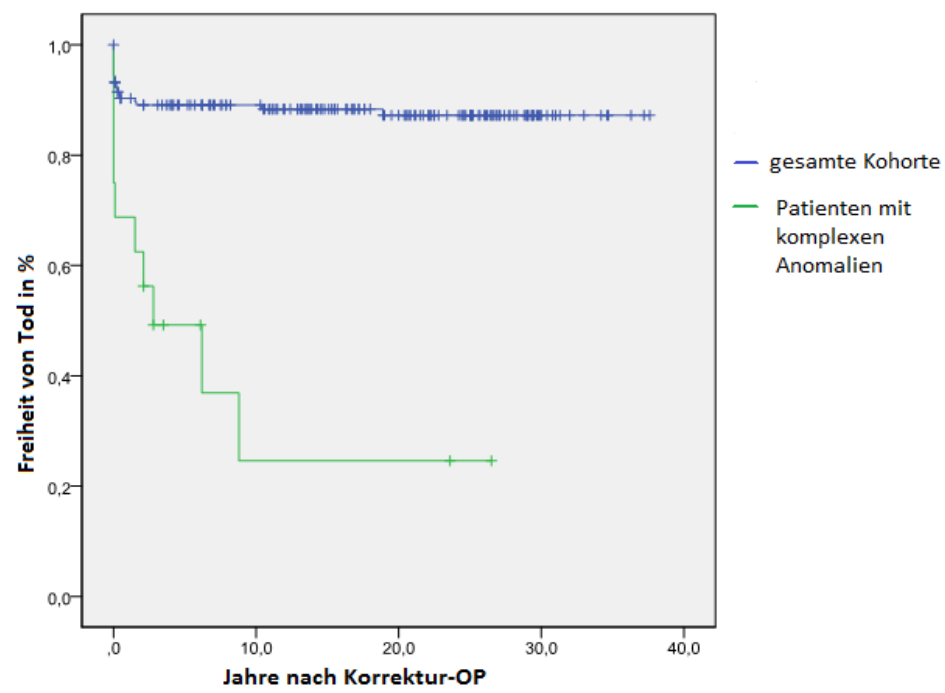

5 Überlebenskurve nach Kaplan Meier Vergleich isoliert/komplex

\begin{tabular}{l|l}
$\begin{array}{l}\text { Jahre } \\
\text { nach } \\
\text { Korrek- } \\
\text { tur }\end{array}$ & $\begin{array}{l}\text { Überlebenswahrschein- } \\
\text { lichkeit }\end{array}$ \\
\hline 1 & $68,8 \pm 11,6 \%$ \\
5 & $49,2 \pm 12,7 \%$ \\
10 & $24,6 \pm 13,8 \%$
\end{tabular}

Zur besseren Übersicht hier eine genaue Aufschlüsselung der Patienten mit komplexen Anomalien und den jeweiligen Diagnosen

\begin{tabular}{|lcl|}
\hline Assoziierte Fehlbildung & Anzahl Patienten & $\begin{array}{l}\text { Anzahl Überle- } \\
\text { bender (ggf Alter im } \\
\text { Jahr 2012 in Jahren) }\end{array}$ \\
\hline $\begin{array}{l}\text { single ventricle, isoliert } \\
\text { Heterotaxie, isoliert }\end{array}$ & 4 & 0 \\
\hline $\begin{array}{l}\text { single ventricle + Heterota- } \\
\text { xie }\end{array}$ & 1 & 0 \\
\hline VSD & 6 & $2(2$ und 3) \\
\hline VSD + TGA & 2 & $1(26)$ \\
\hline Pulmonalklappenstenose & 1 & $1(3)$ \\
\hline Cor triatriatum & 1 & $1(6)$ \\
\hline
\end{tabular}

Tabelle 15 Aufschlüsselung der Patienten mit komplexen Fehlbildungen und Anzahl der Überlebenden

Die Aufschlüsselung der Patienten mit komplexen Herzfehlern (siehe Tabelle 16 Tab..) zeigt eine Vielfalt verschiedener assoziierter Fehlbildungen. Für die zwei größten Gruppen (univentrikuläre Malformationen, Heterotaxie-Syndrom) berechneten sich separat folgende Überlebenswahrscheinlichkeiten:

\subsubsection{TAPVC + single ventricle}

Diese Untergruppe zeigte bereits nach einem Jahr nur noch eine Überlebenswahrscheinlichkeit von $54,5 \pm 15,0 \%$. Nach fünf Jahren halbiert sich die Überlebenswahrscheinlichkeit und nach 10 Jahren ist sie bereits $0 \%$. 


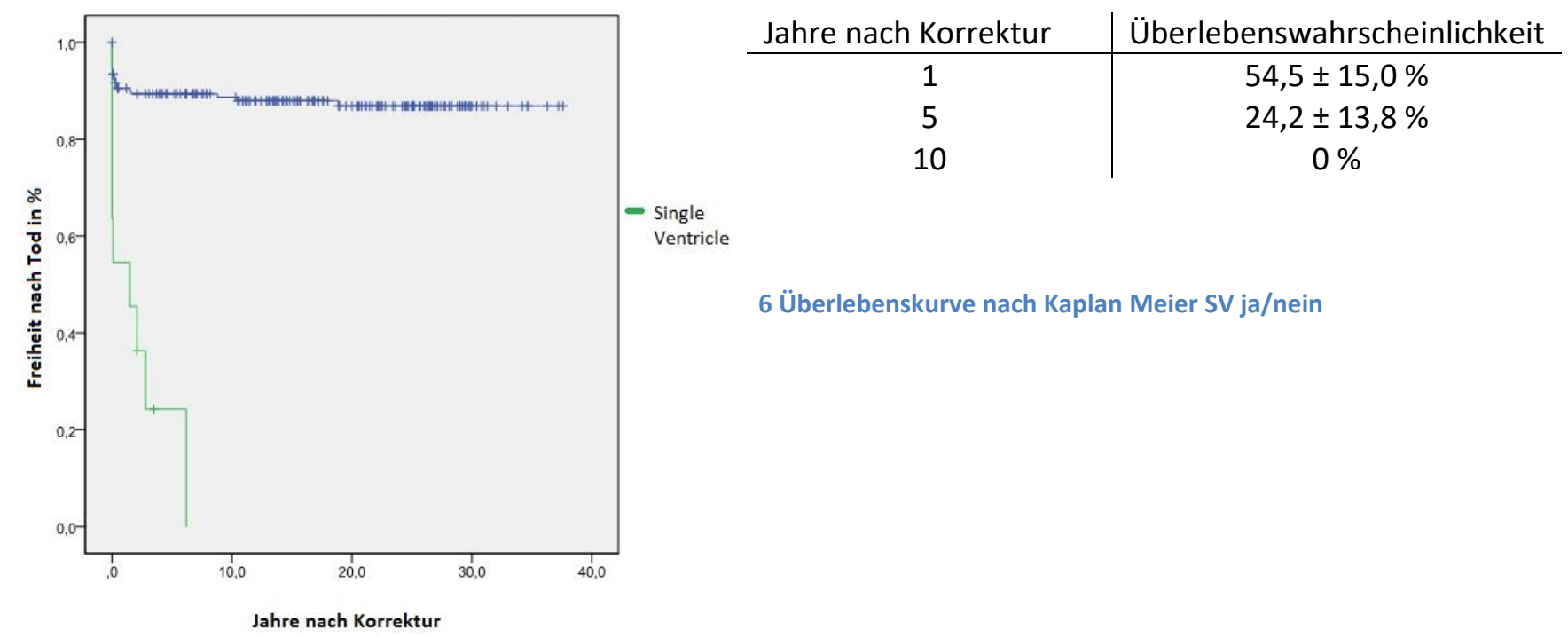

\subsubsection{TAPVC + Heterotaxie}

Auch wenn hier die Überlebenswahrscheinlichkeit gegenüber derer mit lediglich univentrikulären Formationen ohne Heterotaxie-Syndrom im ersten Jahr noch höher scheint, beträgt auch hier die Wahrscheinlichkeit bis 10 Jahre nach der Operation zu überleben gleich $0 \%$.
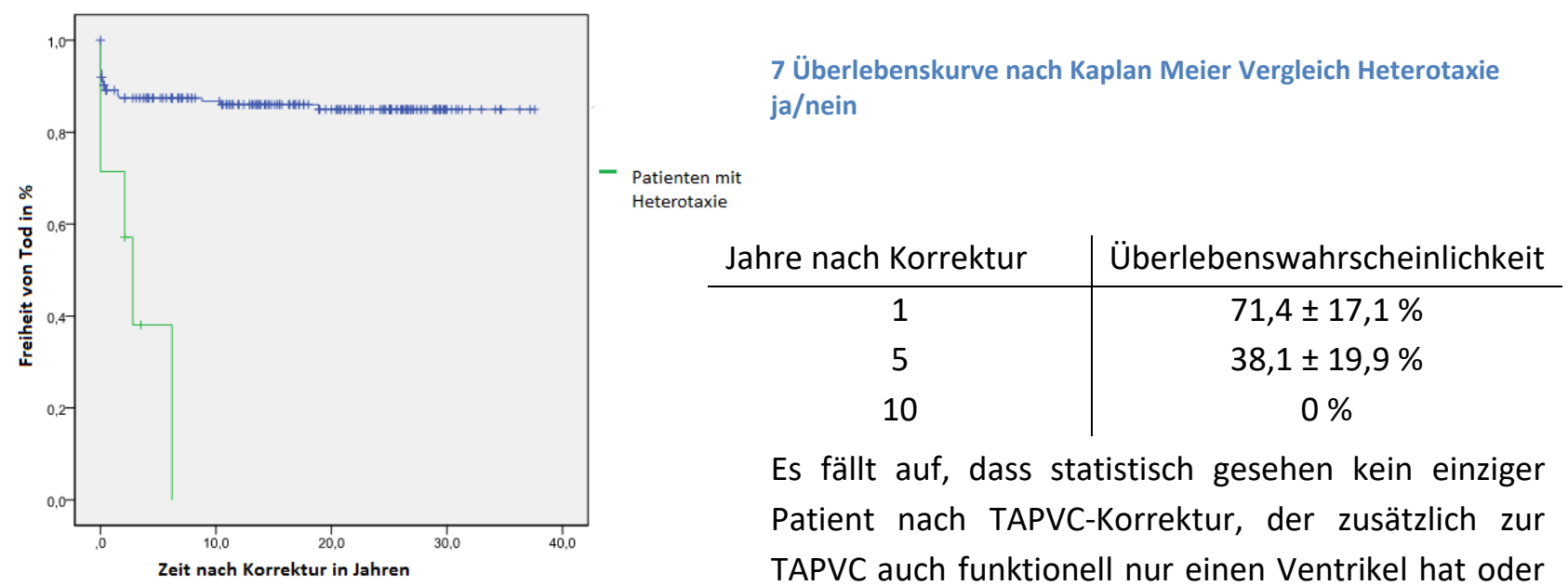

Es fällt auf, dass statistisch gesehen kein einziger Patient nach TAPVC-Korrektur, der zusätzlich zur TAPVC auch funktionell nur einen Ventrikel hat oder das Heterotaxie-Syndrom aufweist, das zehnte Lebensjahr erreicht. Dazu muss allerdings erwähnt werden, dass in unserem Patientengut zwei der Patienten, die zusätzlich ein Heterotaxie-Syndrom bzw. univentrikuläre Malformationen haben, zwar zum jetzigen Zeitpunkt noch leben, allerdings noch nicht zehn Jahre alt sind. 


\section{Auswertung}

Zunächst wurden die Daten aller 193 Patienten analysiert. Anschließend erfolgte dieselbe Analyse für die Patienten, die eine isolierte TAPVC aufwiesen, wozu die 16 Patienten mit zusätzlichen kardialen Malformationen aus der Analyse ausgeschlossen wurden. Diese 16 Patienten wurden als separate Gruppe der gleichen Analyse unterzogen.

\subsection{Risikofaktoren für Letalität}

Zur Identifizierung von Risikofaktoren für die Letalität wurden folgende Daten auf ihren Einfluss getestet:

1. Demographische Daten und TAPVC-Typ
a. Geschlecht
b. suprakardialer Typ
c. kardialer Typ
d. infrakardialer Typ
e. gemischter Typ

2. präoperative Situation

a. Komplexität des Vitiums (single ventricle, Heterotaxie-Syndrom)

b. Präoperative pulmonalvenöse Obstruktion

3. Operative Daten
a. Operationsjahr (vor/nach 1990)
b. Alter bei Operation $(</>30$ Tage)
c. Nahtmaterial (resorbierbar/nicht resorbierbar)
d. Totaler Kreislaufstillstand

\subsubsection{Analyse der gesamten Kohorte}

Inkludiert wurden alle 193 Patienten. Die univariate Analyse erbrachte folgende Ergebnisse.

\begin{tabular}{llll} 
Variable & Anzahl & Ereignisse & p-Wert univariat \\
\hline $\begin{array}{c}\text { Geschlecht } \\
- \text { Männlich }\end{array}$ & 109 & 16 & 0,527 \\
- weiblich & 84 & 15 & \\
\hline
\end{tabular}

Konnektionstyp 


\begin{tabular}{llll}
\hline- suprakardialer Typ & 89 & 19 & 0,079 \\
- Kardialer Typ & 34 & 5 & 0,855 \\
$-\quad$ Infrakardialer Typ & 59 & 7 & 0,324 \\
- Gemischter Typ & 11 & 0 & 0,154 \\
\hline PVO & $\mathbf{1 0 5}$ & $\mathbf{2 2}$ & $\mathbf{0 , 0 4 4}$ \\
\hline Komplexe Anomalie & $\mathbf{1 6}$ & $\mathbf{1 0}$ & $<0,001$ \\
\hline Univentrikuläre Malformation & $\mathbf{1 1}$ & $\mathbf{9}$ & $<0,001$ \\
\hline Heterotaxie & $\mathbf{7}$ & $\mathbf{5}$ & $<0,001$ \\
\hline OP-Daten & & & \\
- OP-Alter <30 Tage & 103 & 19 & 0,316 \\
- OP-Zeitpunkt <1990 & 102 & 18 & 0,349 \\
$-\quad$ Kreislaufstillstand & 47 & 5 & 0,373 \\
- Nicht-resorb. Naht & 40 & 8 & 0,403 \\
\hline
\end{tabular}

Tabelle 17 Ergebnisse der univariaten Testung für erhöhte Letalität (gesamte Kohorte)

Aus diesen Daten ist ersichtlich, dass das zusätzliche Vorhandensein weiterer kardialen Anomalien (vor allem univentrikuläre Anomalien und Heterotaxie-Syndrom) einen statistisch relevanten Einfluss auf die Überlebenswahrscheinlichkeit hat. Des Weiteren bestimmt auch eine vorhandene präoperative Obstruktion die Letalitätsrate. Keine der anatomische Variablen zeigte sich als statistisch relevante Einflussgröße. Für den suprakardialen Konnektionstyp zeigt sich zwar ein Trend zu geringerer Überlebenswahrscheinlich, statistische Relevanz wurde allerdings nicht erreicht.

Diese Variablen, die in der univariaten Analyse statistische Relevanz erreichten, wurden in die anschließende multivariate Analyse miteinbezogen. Diese lieferte folgende Ergebnisse:

\begin{tabular}{llll} 
Variable & $\mathbf{p}$-Wert univariat & $\mathbf{p}$-Wert multivariat & Hazard ratio \\
\hline Komplexe Anomalie & $<0,001$ & $>0,1$ & \\
\hline $\begin{array}{l}\text { Univentrikuläre Malfor- } \\
\text { mation }\end{array}$ & $<0,001$ & $<0,001$ & $\mathbf{9 . 7}(\mathbf{4 . 3 - 2 1 . 8 )}$ \\
\hline Heterotaxie & $<0,001$ & $>0,1$ & \\
\hline PVO &, 044 & 0,097 & $1.9(0.8-4.2)$ \\
\hline
\end{tabular}

Tabelle 18 Ergebnisse der multivariaten Testung für erhöhte Letalität (gesamte Kohorte)

Eine komplexe präoperative Situation verringert die Überlebenswahrscheinlichkeit. Die zusätzliche Diagnose einer univentrikulären Malformation ist ein signifikanter Risikofaktor für eine höhere Letalität, sowohl in der univariaten, als auch in der multivariaten Analyse. Auch eine präoperativ bestehende Obstruktion der Pulmonalvenen korreliert mit der langfristigen postoperativen Überlebenswahrscheinlichkeit. Statistische Relevanz auf dem 5\% Niveau wurde allerdings keine erreicht.

\subsubsection{Analyse der Patienten mit isolierter TAPVC}

Nachdem die oben aufgeführte Analyse zeigt, dass komplexe Anomalien eindeutig mit geringerer Überlebenswahrscheinlichkeit korrelieren, wurde eine zweite Analyse zum Abschätzen der Risikofaktoren bei Patienten mit isolierter TAPVC, also ohne zusätzliche Fehlbildungen, durchgeführt. Dazu 
wurden die Patienten, die komplexe Anomalien aufwiesen ( $n=16)$ exkludiert und die Daten der verbleibenden ( $n=177$ ) Patienten nach dem oben beschriebenen Schema analysiert.

\begin{tabular}{llll} 
Variable & Anzahl & Ereignisse & p-Wert univariat \\
\hline $\begin{array}{l}\text { Geschlecht } \\
-\quad \text { Männlich }\end{array}$ & 103 & & 0,879 \\
$\quad$ weiblich & 74 & & \\
\hline Konnektionstyp & & & \\
$-\quad$ suprakardialer Typ & 89 & 19 & 0,224 \\
$-\quad$ Kardialer Typ & 34 & 5 & 0,366 \\
$-\quad$ Infrakardialer Typ & 59 & 7 & 0,972 \\
$-\quad$ Gemischter Typ & 11 & 0 & 0,238 \\
$\quad$ & & & \\
\hline PVO & $\mathbf{1 0 5}$ & $\mathbf{2 2}$ & $\mathbf{0 , 0 3 0}$ \\
\hline OP-Daten & & & \\
$-\quad$ OP-Alter <30 Tage & 103 & 19 & 0,413 \\
$-\quad$ OP-Zeitpunkt <1990 & 102 & 18 & 0,376 \\
$-\quad$ Kreislaufstillstand & $\mathbf{4 7}$ & $\mathbf{5}$ & $\mathbf{0 , 0 1 7}$ \\
$-\quad$ Nicht-resorb. Naht & 40 & 8 & 0,079 \\
\hline
\end{tabular}

Tabelle 19 Ergebnisse der univariaten Testung für erhöhte Letalität (Patienten mit isolierter TAPVC)

Als statistisch relevante Risikofaktoren für erhöhte Letalität in der Gruppe der Patienten mit isolierter TAPVC zeigte sich eine präoperativ bestehende pulmonale Obstruktion, sowie die Durchführung der Korrekturoperation bei besser mit totalem Kreislaufstillstand und besser in tiefer Hypothermie. Das dabei verwendete Nahtmaterial zeigt eine Korrelation zu erhöhter Letalität, statistische Relevanz wurde aber nicht erreicht.

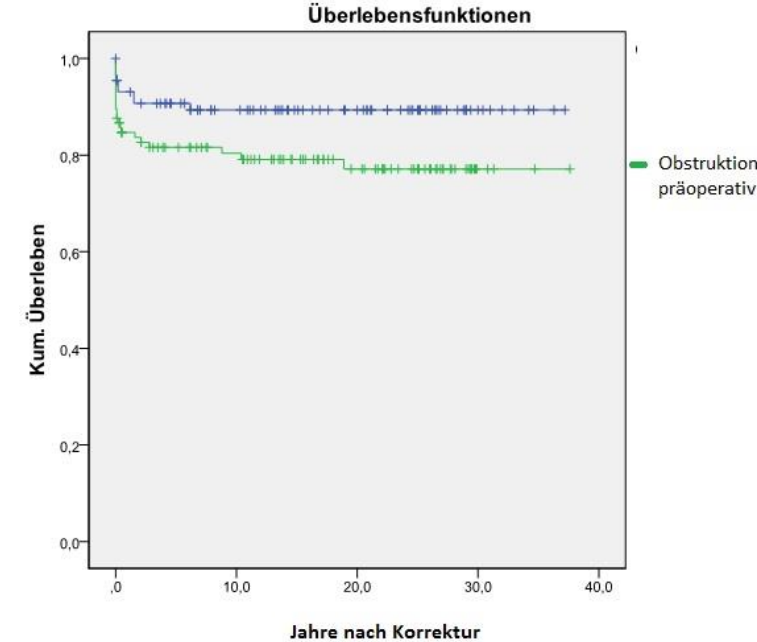

8 Kaplan-Meier - Überlebenskurve für Patienten mit (blau) und ohne (grün) PVO

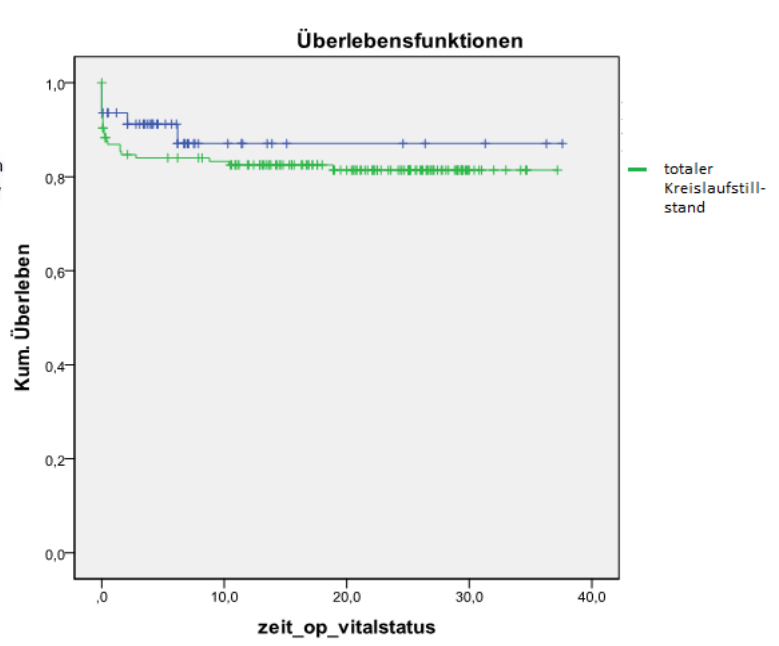

9 Kaplan-Meier - Überlebenskurve für Patienten ohne(blau) und mit (grün) Kreislaufstillstand

Zusammenfassend ergibt dies folgende Risikofaktoren für die Letalität: 


\begin{tabular}{ccc}
\hline Getestete Faktoren & $\begin{array}{c}\text { Relevanter Risikofaktor } \\
\text { Gesamte Kohorte }\end{array}$ & $\begin{array}{c}\text { Relevanter Risikofaktor } \\
\text { Gruppe TAPVC isoliert }\end{array}$ \\
\hline Alter & Nein & Nein \\
\hline Geschlecht & Nein & Nein \\
\hline Alter bei Korrektur-OP (s30 Tage) & Nein & Nein \\
\hline TAPVC-Typ & Nein & Nein \\
\hline Single ventricle & JA ( $p$-Wert <0,001) & Nein \\
\hline Heterotaxie-Syndrom & Nein & Nein \\
\hline Pulm. Hypertonie & Nein & JA (p-Wert 0,030) \\
\hline Präop. Obstruktion & Nein & Nein \\
Operationsjahr vor/nach 1990 & Nein & Nein \\
\hline Nahtmaterial & Nein & JA ( $p$-Wert 0,017) \\
\hline Kreislaufstillstand & Nein \\
\hline
\end{tabular}

Tabelle 20 Übersicht relevanter Risikofaktoren für Letalität

\subsection{Risikofaktoren für Re-Operationen wegen postoperativer PVO}

Der Einfluss auf Re-Operationen aufgrund postoperativ entstandener pulmonalvenöser Obstruktion wurden für folgende Variablen analysiert:

4. Demographische Daten und TAPVC-Typ
a. Geschlecht
b. suprakardialer Typ
c. kardialer Typ
d. infrakardialer Typ
e. gemischter Typ

5. präoperative Situation
a. Komplexität des Vitiums (single ventricle, Heterotaxie-Syndrom)
b. Präoperative pulmonalvenöse Obstruktion

6. Operative Daten
a. Operationsjahr (vor/nach 1990)
b. Alter bei Operation $(</>30$ Tage)
c. Nahtmaterial (resorbierbar/nicht resorbierbar)
d. Totaler Kreislaufstillstand

\subsubsection{Analyse der gesamten Kohorte}




\begin{tabular}{llll}
\hline Geschlecht & & & \\
$-\quad$ Männlich & 109 & 9 & 0,601 \\
$-\quad$ weiblich & 84 & 5 & \\
\hline Konnektionstyp & & & \\
\hline$\quad$ suprakardialer Typ & 89 & 5 & 0,529 \\
$-\quad$ Kardialer Typ & 34 & 4 & 0,278 \\
$-\quad$ Infrakardialer Typ & 59 & 4 & 0,789 \\
$-\quad$ Gemischter Typ & 11 & 1 & 0,921 \\
$\quad$ & & & \\
$\quad$ & 105 & 6 & 0,701 \\
\hline PVO & 16 & 2 & 0,118 \\
\hline Komplexe Anomalie & 11 & 1 & 0,225 \\
\hline Univentrikuläre Malformation & 7 & 0 & 0,561 \\
\hline Heterotaxie & & & \\
\hline OP-Daten & 103 & 12 & 0,007 \\
$-\quad$ OP-Alter <30 Tage & 102 & 6 & 0,346 \\
$-\quad$ OP-Zeitpunkt <1990 & 47 & 13 & 0,531 \\
$-\quad$ Kreislaufstillstand & 40 & 5 & 0,452 \\
$-\quad$ Nicht-resorb. Naht &
\end{tabular}

Tabelle 21 Übersicht der Ergebnisse der Analyse der gesamten Kohorte auf Notwendigkeit von TAPVC-Re-OPs

Die statistische Auswertung der potentielle Einflussfaktoren auf die Notwendigkeit von ReOperationen auf Grund postoperativ entstandener Stenosen an der Korrekturanastomose der TAPVC zeigte als einzigen statistisch relevanten Risikofaktor die Neugeborenenperiode ( $<30$ Tage) bei Korrektur-OP. Von 14 Patienten, die später re-operiert werden mussten, waren 12 Patienten bei der initialen Korrektur Neugeborene. In der multivariaten Analyse errechnete sich ein p-Wert von 0,007 und zeigte somit statistische Relevanz.

\subsubsection{Analyse der Patienten mit isolierter TAPVC}

\begin{tabular}{|c|c|c|c|}
\hline Variable & Anzahl & Ereignisse & $\mathrm{p}$-Wert univariat \\
\hline \multicolumn{4}{|l|}{ Geschlecht } \\
\hline - Männlich & 103 & 8 & 0,567 \\
\hline - weiblich & 74 & 4 & \\
\hline \multicolumn{4}{|l|}{ Konnektionstyp } \\
\hline - suprakardialer Typ & 89 & 4 & 0,440 \\
\hline - Kardialer Typ & 34 & 3 & 0,422 \\
\hline - Infrakardialer Typ & 59 & 4 & 0,976 \\
\hline - Gemischter Typ & 11 & 1 & 0,767 \\
\hline
\end{tabular}

\begin{tabular}{llll}
\hline PVO & 105 & 6 & 0,636 \\
\hline$\underline{\text { OP-Daten }}$
\end{tabular}


- OP-Alter <30 Tage

- OP-Zeitpunkt <1990

- Kreislaufstillstand

- Nicht-resorb.Naht

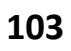

102

47

40
10

6

5

8
0,022

0,567

0,863

0,334

Tabelle 22 Übersicht der Ergebnisse der Analyse der Untergruppe an Patienten mit isolierter TAPVC auf Notwendigkeit von TAPVC- Re-OPs

In der gesonderten Analyse der Gruppe an Patienten mit isolierter TAPVC zeigte sich junges Patientenalter (<30 Tage) bei Korrektur ebenfalls als einziger Risikofaktor für die Notwendigkeit einer späteren Re-OP zur Korrektur einer postoperativ aufgetretenen PVO. Von den 12 Patienten mit isolierter TAPVC, die eine Re-Operation benötigten waren 10 Patienten bei der initialen Korrektur Neugeborene.

\subsection{Risikofaktoren für kardiale Re-Operationen wegen ande- re Indikation}

Nachdem unter den Re-Operationen auch Operationen waren, welche nicht die Anastomose der Pulmonalvenen betrafen, sondern andere kardiale Diagnosen behoben, erfolgte eine weitere Analyse mit der Frage nach Risikofaktoren für kardiale Re-Operationen aus anderer Indikation. Auch diese wurde für die gesamte Kohorte und die Untergruppe derer mit isolierter TAPVC durchgeführt.

\subsubsection{Analyse der gesamten Kohorte}

\begin{tabular}{|c|c|c|c|c|}
\hline Variable & Anzahl & Ereignisse & p-Wert univariat & $\mathrm{p}$-Wert multivariat \\
\hline \multicolumn{5}{|l|}{ Geschlecht } \\
\hline - Männlich & 109 & 9 & 0,783 & \\
\hline - weiblich & 84 & 7 & & \\
\hline \multicolumn{5}{|l|}{ Konnektionstyp } \\
\hline - suprakardialer Typ & 89 & 11 & 0,396 & \\
\hline - Kardialer Typ & 34 & 3 & 0,328 & \\
\hline - Infrakardialer Typ & 59 & 1 & 0,084 & \\
\hline - Gemischter Typ & 11 & 1 & 0,946 & \\
\hline PVO & 105 & 2 & 0,837 & \\
\hline Komplexe Anomalie & 16 & 6 & $<0,001$ & \\
\hline \multirow[t]{3}{*}{ Univentrikuläre Malformation } & 11 & 6 & $<0,001$ & $<0,001$ \\
\hline & & & & hazard rate \\
\hline & & & & $165,5(10,3-2645,8)$ \\
\hline Heterotaxie & 7 & 5 & $<0,001$ & \\
\hline
\end{tabular}




$\begin{array}{llll}\text { - OP-Alter }<30 \text { Tage } & 103 & 7 & 0,118 \\ \text { - OP-Zeitpunkt }<1990 & 102 & 5 & \mathbf{0 , 0 1 8} \\ \text { - Kreislaufstillstand } & 47 & 10 & 0,154 \\ \text { - Nicht-resorb. Naht } & 40 & 4 & 0,886\end{array}$

Tabelle 23 Übersicht der Ergebnisse der Analyse der gesamten Kohorte auf Notwendigkeit für andere kardiale Re-OPs

In der univariaten Analyse der Faktoren zeigt der Faktor komplexes Herzvitium einen p-Wert <0,05. Zusätzlich zeigte auch die chirurgische Ära (OP-Zeitpunkt <1990) einen $p$-Wert $<0,05$. Somit flossen die Faktoren "komplexe Anomalien“, "univentrikuläre Malformationen“, „Heterotaxie“ und "OpZeitpunkt<1990" in die multivariate Analyse ein.

Hier zeigte sich als einzig relevanter Risikofaktor für kardiale Re-OPerationen, welche nicht die Lungenvenen betrafen das Vorhandensein einer zusätzlichen univentrikulären Malformation.

\subsubsection{Analyse der Patienten mit isolierter TAPVC}

In der Analyse der Untergruppe von Patienten mit isolierter TAPVC fand sich kein statistisch relevanter Risikofaktor für die Notwendigkeit kardialer Re-Operationen. Bereits in der univariaten Analyse wurde für keinen der oben angeführten Faktoren eine statistische Relevanz erreicht.

Zusammenfassend ergaben sich folgende Risikofaktoren für Re-Operationen:

\begin{tabular}{|c|c|c|c|c|}
\hline \multirow[t]{2}{*}{ Getestete Faktoren } & \multicolumn{2}{|c|}{ Risikofaktor Re-OP-TAPVC } & \multicolumn{2}{|c|}{ Risikofaktor andere kard. Re-OP } \\
\hline & Ges. Kohorte & TAPVC isoliert & Ges. Kohorte & TAPVC isoliert \\
\hline Alter & Nein & Nein & Nein & Nein \\
\hline Geschlecht & Nein & Nein & Nein & Nein \\
\hline Alter bei Korrektur-OP ( $\leq 30$ Tage) & Ja (p 0.007) & $\mathrm{Ja}(\mathrm{p} 0,022)$ & Nein & Nein \\
\hline TAPVC-Typ & Nein & Nein & Nein & Nein \\
\hline Single ventricle & Nein & Nein & $\mathrm{Ja}(\mathrm{p}<0.001)$ & Nein \\
\hline Heterotaxie-Syndrom & Nein & Nein & Nein & Nein \\
\hline Pulm. Hypertonie & Nein & Nein & Nein & Nein \\
\hline Präop. Obstruktion & Nein & Nein & Nein & Nein \\
\hline Operationsjahr & Nein & Nein & Nein & Nein \\
\hline Nahtmaterial & Nein & Nein & Nein & Nein \\
\hline Kreislaufstillstand & Nein & Nein & Nein & Nein \\
\hline
\end{tabular}

Tabelle 24 Übersicht Risikofaktoren für Re-Operationen 


\section{Diskussion}

\subsection{Studiengröße und Patientenauswahl}

In dieser Studie wurden Daten von insgesamt 193 Patienten ausgewertet. Das sind Daten von sämtlichen Patienten, bei denen eine angeborene Lungenvenenfehlkonnektion im Zeitraum zwischen 1974 und 2011 am Deutschen Herzzentrum München (DHZ) korrigiert wurde. Dabei wurden ohne Ausnahme alle am DHZ operierten Patienten inkludiert; auch solche, die bereits voroperiert waren (eventuell auch in anderen Kliniken) oder die zusätzliche kardiale Fehlbildungen aufwiesen. Patienten, die an anderen Klinik der Korrektur-OP unterzogen wurden, aber im DHZ weiter betreut werden, wurden nicht in die Studie mit eingeschlossen. Patienten mit partieller Lungenvenenfehlmündungen wurden ebenfalls nicht integriert.

Vergleicht man die Patientenanzahl mit anderen Publikationen zu diesem Herzfehler, so gibt es nur eine monozentrische Studie, welche eine größere Kohorte (377 Patienten) untersucht hat (Karamlou et al., 2007). Allerdings wurden, im Gegensatz zur hier vorliegenden, in dieser Studie auch Patienten mit TAPVC inkludiert, die nicht korrigierend operiert wurden (50 Patienten), sondern bis zum Studienabschluss lediglich konservativ behandelt wurden. Nicht mit eingeschlossen wurden dort Patienten mit schwerwiegenden assoziierten kardialen Fehlbildungen (außer PDA und ASD), was Einfluss nimmt auf die gesamte Sterblichkeit des Patientenguts.

Andere größere Studien zur TAPVC umfassten 178 Patienten, wovon 44 zusätzliche Anomalien aufweisen (Lacour-Gayet et al., 1999) und 170 Patienten, wovon allerdings nur ein Patient eine weitere kardiale Fehlbildung hatte (Caldarone et al., 1998b). Eine rezente multizentrische Studie erfasste Daten von insgesamt 406 Patienten mit TAPVC (Seal et al.). Das ist die bis jetzt größte untersuchte Fallzahl. Dabei gilt es zu beachten, dass durch den Zusammenschluss mehrerer Zentren das Patientenkollektiv keinem homogenen, operativen und perioperativen Management unterzogen wurde, was bei der Interpretation der Daten zu berücksichtigen ist.

Nachdem die TAPVC ein sehr seltener Herzfehler ist, braucht es einen langen Beobachtungszeitraum, um Daten zu einem Kollektiv zu sammeln, deren Auswertung aussagekräftige Konklusionen zulässt. Das erklärt, warum die meisten anderen hier zitierten Studien geringe Fallzahlen inkludieren, was bei der Relevanz der Aussagen immer bedacht werden sollte.

Mit einer durchschnittlichen Follow-up-Zeit von 15 Jahren (längstes Follow-up 38 Jahre) liegt die hier vorliegende Studie allen oben genannten Studien weit voraus, womit vor allem der Beurteilung des Langzeitverlaufs nach Korrektur in dieser Arbeit eine besonderes Gewicht zukommt. Bei der folgenden Diskussion der Risikofaktoren sei also beachtet, dass die Vergleichbarkeit mit anderen Studien eingeschränkt ist, da in der hier vorliegenden Studien durch den längeren Beobachtungszeitraum mehr Spätkomplikationen bzw. Spätletalitäten mitbeachtet wurden.

\subsection{Demographische Daten}

Zur Inzidenz der TAPVC bei Lebendgeburten kann in dieser Studie keine Aussage getroffen werden, denn die Patienten dieser Kohorte stammen aus keiner definierten Bevölkerungsmenge. Laut Litera- 
tur beträgt sie 1 bis 3\% aller angeborener kardialer Fehlbildungen (Michielon et al., 2002). Die Geschlechterverteilung verhielt sich in dieser Studie weiblich zu männlich wie 1:1,3. Das entspricht der Verteilung vergleichbarer Studien (Caldarone et al., 1998b; Husain et al., 2012; Karamlou et al., 2007). Auffällig ist, dass die Geschlechterverteilung in unserer Untergruppe der Patienten mit zusätzlichen Anomalien deutlich auf Seite des weiblichen Geschlechts liegt (1:2,2). Größere Studien zu dieser Patientengruppe zeigen diesen Unterschied nicht (Gaynor et al., 1999). Aufgrund der geringen Fallzahl dieser Untergruppe, ist vermutlich davon auszugehen, dass es sich um eine zufällige Häufung handelt.

\subsection{Präoperative Situation}

Die präoperative Situation, die das chirurgische Vorgehen bestimmt, wird gekennzeichnet durch den TAPVC-Typ, das Vorhandensein einer Obstruktion der Pulmonalgefäße, das Vorhandensein zusätzlicher kardialer Fehlbildungen und eventuell vorher durchgeführte Eingriffe.

\subsubsection{TAPVC-Typen}

Die Häufigkeitsverteilung der verschiedenen TAPVC-Typen wird in der Literatur mit 45\% suprakardialem, 25\% kardialem, 25\% infrakardialem und 5\% gemischtem Konnektionstyp angegeben (Bharati \& Lev, 1973; Craig et al., 1957; Delisle et al., 1976).

Das hier vorliegende Patientengut weist im Vergleich dazu einen weitaus höheren Anteil an infrakardial konnektierenden TAPVCs auf, bei einem geringen Anteil an kardialen Konnektionstypen. Nachdem Patienten mit infrakardialer TAPVC grundsätzlich bereits präoperativ eine Obstruktion aufweisen, ist nicht überraschend, dass auch in unserem Kollektiv der Anteil an Patienten mit PVO im Vergleich zu anderen Studien erhöht ist.

In der Literatur wird der Zusammenhang zwischen TAPVC-Konnektionstyp und Überlebenswahrscheinlichkeit kontrovers diskutiert. Einig ist man sich (Hyde et al., 1999; Karamlou et al., 2007), dass der infrakardiale Konnektionstyp immer mit einer präoperativen pulmonalvenösen Obstruktion korreliert, was sich mittlerweile zu einer gängigen Lehrmeinung etabliert hat (Anderson, 2010). Diese Obstruktion ist bedingt durch die Einmündung des Sammelgefäßes in die Vena portae.

Andere Autoren beobachten auch einen Zusammenhang zwischen suprakardialem Konnektionstyp und präoperativer Obstruktion. In der Kohorte von Hyde et al. weisen $28 \%$ der Patienten mit suprakardialer Lungenvenenkonnektion schon präoperativ eine Obstruktion auf (Hyde et al., 1999), in der Studie von Husain et al. 23,1\%(Husain et al., 2012). Auch in unserer Studie zeigte sich ein ähnlich hoher Anteil an Patienten mit suprakardialem Konnektionstyp und präoperativer Obstruktion $(21,2 \%)$. In der statistischen Analyse ließ sich eine Korrelation zwischen suprakardialer Konnektion und erhöhter Sterblichkeit feststellen. Statistische Relevanz hierfür wurde aber nicht erreicht $(p=0,79)$.

Das Einhergehen einer erhöhten Letalität mit einem gemischten Konnektionstyp, wie es von anderen Autoren (Delius et al., 1996; Kirshbom et al., 2002) beschrieben wird, konnten wir nicht bestätigen. Allerdings wiesen nur 6\% (10 Patienten) unserer Kohorte diesen Typ auf, womit es sein kann, dass aufgrund der niedrigen Zahl die Aussagekraft eingeschränkt ist. 


\subsubsection{Obstruktion}

Die bei 54\% unserer Patienten schon präoperativ bestehende pulmonalvenöse Obstruktion lässt sich in den meisten Fällen (76\%) auf den infrakardialen Konnektionstyp zurückführen, was typisch für diesen Typ ist (Anderson, 2010)

Auch wenn einige andere Studien Kohorten mit einem hohen Anteil an Patienten mit PVO von bis zu 55\% beschreiben (Hancock Friesen et al., 2005; Seale et al., 2013), weist das hier beschriebene Kollektiv mit 54\% einen höheren Prozentsatz an Patienten mit Obstruktion auf als die sonst in der Literatur angegebenen Prozentangaben zwischen 25,5\% und 31\% (Husain et al., 2012; Hyde et al., 1999; Karamlou et al., 2007). Dies ist dadurch zu erklären, dass ein weitaus höherer Anteil an Patienten unserer Kohorte eine Konnektion vom infrakardialen Typ aufwies.

Nachdem in der Analyse der hier vorliegenden Daten eine präoperativ bestehende Obstruktion als Risikofaktor für erhöhte Sterblichkeit isoliert wurde, wird der Zusammenhang zwischen präoperativer Obstruktion und Sterblichkeit später ausführlicher diskutiert.

\subsection{3 „Komplexe“ Herzfehler}

Die in diese Studie integrierten Patienten, die neben einer TAPVC noch weitere kardiale Fehlbildungen hatten, bilden mit 8,3\% eine relativ kleine Gruppe, die aber aufgrund ihrer schlechten Prognose (Hammon et al., 1980) besonderes Augenmerk verdient. In den meisten größeren Studien wurden Patienten mit kardialen Zusatzdiagnosen ausgeschlossen. Nur wenige Autoren beschreiben Kohorten, in denen auch Patienten mit weiteren zusätzlichen kardialen Malformationen inkludiert sind (Caldarone et al., 1998b; Gaynor et al., 1999; Heinemann et al., 1994; Husain et al., 2012). Ähnlich wie bei diesen Autoren sind auch in der hier vorliegenden Studie zusätzliche Malformationen mit univentrikulärem Herzen am häufigsten, besonders die, die im Rahmen eines Heterotaxie-Syndroms auftreten (Heinemann et al., 1994). Dieses hat unter den funktionell univentrikulären Malformationen per se ein schlechte Prognose, besonders aber in Kombination mit einer TAPVC (Caldarone et al., 1998b; Heinemann et al., 1994; Yoshimura et al., 2001).

Unter den Begriff des univentrikulären Herzens fällt eine Gruppe kardialer Anomalien, wie das Hypoplastische-Linksherz-Syndrom, die Trikuspidalklappenatresie und andere Fehlbildungen, bei denen das Herz funktionell nur einen Ventrikel hat, das sowohl den pulmonalen als auch den systemischen Kreislauf mit Mischblut speist - die Kreisläufe sind parallel geschaltet. Dabei kann entweder der rechte oder der linke Ausflusstrakt unterentwickelt sein, wodurch es entweder zu einer Lungenminderoder zu einer Lungenhyperperfusion kommt. Bei Geburt ist entweder die Lungenperfusion oder der Systemkreislauf abhängig von einem offenen Ductus arteriosus. Damit gilt es, als erste Maßnahme, die balancierte Perfusion beider Kreisläufe dauerhaft zu gewährleisten. Die Lungenperfusion wird dabei mit einem aortopulmonaler Shunt sichergestellt. Diese Shuntanlage erfolgte bei den betroffenen Patienten aus unserem Kollektiv gleichzeitig mit der Korrektur der Lungenvenenfehlmündung.

Auch wenn die Anatomie des Univentrikulären Herzen verschieden sein kann, ist die gemeinsame Endstrecke der Therapie meist eine komplette Fontan-Operation (de Leval, 2005), in der zwei in Serie geschaltete Kreisläufe entstehen; einer zur Blutoxygenierung über die Lunge, ohne vorgeschaltete Herzkammer, der andere zur systemischen Blutversorgung über die Aorta. Diese Zirkulation entsteht meist in zwei weiteren Operationsschritten. Als erstes wird eine partielle cavopulmonale Konnektion 
(Verbindung der oberen Hohlvene mit den Lungenarterien) hergestellt, der eine erste Volumenentlastung des singulären Ventrikels darstellt. Dieser Schritt sollte wegen des in den ersten Lebensmonaten noch erhöhten pulmonalvaskulären Widerstandes nicht vor dem 4. Lebensmonat durchgeführt werden. Letzter Schritt ist eine totale cavopulmonale Konnektion, mit der dann auch die untere Hohlvene direkt in die Pulmonalarterie mündet. Nachdem hierzu ein Conduit verwendet werden muss, der möglichst mit der adulten Organgröße kompatibel sein soll, erfolgt dieser zweite Schritt meistens zwischen dem 18. und 24. (36.) Lebensmonat (Hofbeck et al., 2000; Kaulitz, Luhmer, Bergmann, Rodeck, \& Hausdorf, 1997; Ovroutski et al., 2009). Einflussfaktoren auf eine erfolgreiche Funktion der Fontan-Zirkulation sind unter anderem der pulmonal-vaskuläre Widerstand und die Größe der Pulmonalgefäße. (Chungsomprasong et al., 2011; Hosein et al., 2007); Parameter, die durch eine Lungenvenenfehlkonnektion ungünstig beeinflusst werden könnten.

Die Analyse unseres Patientenguts zeigt, dass die Kombination mit einer univentrikulärem Malformation einen Risikofaktor für frühzeitiges Ableben bei TAPVC darstellt. Dies wird im Anschluss diskutiert.

\subsubsection{Voroperationen}

Bei unzureichender Mischung von oxygeniertem und venösem Blut muss, bei verschlossenem Foramen ovale als palliative Maßnahme ein Rechts-links-Shunt bis zur endgültigen Korrektion hergestellt werden. In dieser Kohorte war dies nur bei einer Minderheit des Kollektivs notwendig.

Mullins et al (Mullins et al., 1973) befürwortete grundsätzlich eine Ballonatrioseptostomie bei allen Neugeborenen mit TAPVC, in der Annahme das der "natürlich" bestehende Shunt in den meisten Fällen nicht ausreiche. Außerdem fand er in allen untersuchten Patienten eine klinische Besserung, die ein Aufschieben der Korrekturoperation ermöglichte, was die Operationsergebnisse verbessern sollte. Im Gegensatz dazu zeigten Gathman (Gathman \& Nadas, 1970) und Silove (Silove, Behrendt, Aberdeen, \& Bonham-Carter, 1972) in ihren Studien, dass eine Ballonatrioseptostomie bei TAPVC Patienten nur einen geringen Stellenwert hat und behaupten sogar, dass sie, wenn möglich, vermieden werden sollte.

Weniger verallgemeinernde Aussagen trifft Turley (Turley, Tucker, Ullyot, \& Ebert, 1980), der allein den klinischen Zustand als Determinante für eine evtl. Ballonatrioseptostomie betrachtet. Diese Herangehensweise ist auch im Deutschen Herzzentrum München schon lange etabliert.

\subsection{Korrekturoperation}

Seit den 1960er Jahren werden Patienten mit TAPVC korrigierend operiert, wobei Operationen in der ersten Dekade noch sehr hohe Letalitätsraten aufwiesen (33 bis 89\% (Di Eusanio, Sandrasagra, Donnelly, \& Hamilton, 1978; Mustard \& Dolan, 1957)).

Aufgrund der Rarität der TAPVC benötigt man für Studien mit aussagekräftigen Fallzahlen lange Beobachtungszeiträume. Viele Autoren solcher Studien beobachteten im Laufe ihres Beobachtungszeitraumes Veränderungen der Sterblichkeitsraten, die sie den sich fortentwickelnden peri- und postoperativen Medizin zuschreiben. So sehen viele Autoren (Caldarone et al., 1998b; Karamlou et al., 2007; Lamb et al., 1988; Lupinetti, Kulik, Beekman, Crowley, \& Bove, 1993; Oelert, Schafers, Stegmann, Kallfelz, \& Borst, 1986; Sano et al., 1989; Serraf et al., 1991; Wukasch, Deutsch, Reul, Hallman, \& Cooley, 1975), eine Ära-abhängige Verbesserung der Operationsergebnisse (geringere Komplikationsraten und geringere Sterblichkeit) nach Korrekturoperation. In einer multivariaten Ana- 
lyse der hier vorgelegten Daten, lieferte die Untersuchung auf mögliche Abhängigkeit der Sterblichkeit vom Jahr der Korrekturoperation jedoch keinen Zusammenhang. Allgemeine Letalitätsraten blieben über den gesamten Beobachtungszeitraum unverändert.

Im Gegensatz dazu zeigte die Auswertung potentieller Risikofaktoren für kardiale Reoperationen in unserem Patientengut, dass Patienten, bei denen die Korrekturoperation vor 1990 erfolgte, ein statistisch höheres Risiko für kardiale Reoperationen hatten als solche, die nach 1990 operiert wurden. Mögliche Ursachen werden im Kapitel Risikofaktoren diskutiert.

Zu bedenken ist, dass statistische Analysen nicht alle Einflussfaktoren auf Überlebenswahrscheinlichkeit abdecken können. Berücksichtigt werden die bereits oben angeführten objektivierbaren Daten, wie Jahr der Korrektur, Alter und Körpermaße bei Korrektur, Dauer des Kreislaufstillstandes während der Korrektur und Verwendung des Nahtmaterials. Diskutiert werden zudem Fortschritte sowohl im prä-, peri- als auch im postoperativem Management (siehe "Letalität"), welche numerisch nicht greifbar zu machen sind. Auch Unterschiede in der Operationstechnik sind denkbar, aber schwer zu messen und somit schwer zu vergleichen. Des Weiteren sind operative Techniken oft Operateurabhängig. Mueller et al. zeigten, dass eine Sutureless-Technik zur Korrektur der TAPVC sehr gute Ergebnisse liefert. Alle sieben Patienten dieser Studie lebten zum Zeitpunkt des Studienendes und hatten keine postoperative pulmonalvenöse Obstruktion entwickelt, womit er diese Technik als vielversprechende Methode empfiehlt (Mueller, Dave, \& Pretre, 2013). Auch andere Autoren formulierten die Empfehlung einer Sutureless-Technik bereits zur initialen Korrektur (Karamlou et al., 2007; Yun et al., 2005). Bis dato wurde die Sutureless-Technik jedoch nur zur Behebung postoperativ entstandener pulmonalvenöser Stenosen als vorteilhaft belegt (Devaney, Ohye, \& Bove, 2006; LacourGayet, 2006). Fehlende Langzeitergebnisse und sehr geringe erprobte Fallzahlen bei der Rarität der TAPVC verzögern Neuerungen bei operativen Techniken.

Komplizierter ist die Operation bei Patienten mit zusätzlichen Anomalien. In der hier vorliegenden Studie, sowie in der Mehrzahl vergleichbarer Studien, war die initiale Operation immer eine Korrektur der TAPVC. Bei einem Großteil der hier untersuchten Patienten mit komplexeren kardialen Vitien wurden im Rahmen der TAPVC-Korrektur zusätzliche Prozeduren durchgeführt. Dabei handelte es sich entweder um vollständige Korrektur einer zusätzlichen Anomalie, oder um den ersten palliativen Schritt einer mehrzeitigen operativen Versorgung (vor allem Patienten mit univentrikulärem Herz betreffend). Die meisten vergleichbaren Studien beschreiben ein ähnliches Vorgehen, wobei die häufigsten zusätzlichen Prozeduren (ähnlich wie bei dieser Kohorte) Shunt-Anlagen (mBT, AP-Shunt, Norwood I), Septumdefektverschlüsse, Klappenplastiken und Aortenbogenerweiterungen waren (Caldarone et al., 1998b; Hancock Friesen et al., 2005).

Allerdings gibt es einige Autoren, die nicht bei allen Patienten mit komplexen Anomalien initial mehrere Prozeduren kombinierten. Sowohl Gaynor et al. als auch Hancock Friesen et al. zeigen in ihren Arbeiten sogar, dass Patienten, bei denen mehrere Prozeduren gleichzeitig durchgeführt wurden, eine geringere Überlebenswahrscheinlichkeit aufweisen als diejenigen, bei denen Korrekturen unterschiedlicher Anomalien (vor allem single ventricle Physiologie) mehrzeitig, also von der TAPVCKorrektur getrennt, vorgenommen wurden. Gaynor et al. beschreiben sogar, dass die initial isoliert durchgeführte TAPVC-Korrektur ohne gleichtzeitig den ersten Schritt der Fontan-Operatenion ein Risikofaktor für erhöhte Letalität darstellt (Gaynor et al., 1999) .

Ein direkter Vergleich dieser Ergebnisse mit denen unserer Studie ist allerdings nicht möglich, denn unser Studiendesign inkludiert nur Patienten, bei denen immer eine TAPVC-Reparatur durchgeführt 
worden ist. Gaynor et al. allerdings führten nicht bei allen eine Korrektur der TAPVC im Rahmen der ersten Operation durch. Bei 30 Patienten wurden initial andere Korrekturen durchgeführt, weshalb die Vergleichbarkeit hier mit der vorliegenden Studie sehr gering ist.

Die anschließende Tabelle gibt eine Übersicht über die zusätzlich durchgeführten, operativen Maßnahmen im Rahmen der Korrekturoperation der TAPVC in verschiedenen Fallserien. Diese zeigt, dass die meisten zusätzlichen Palliativoperationen bei univentrikulären Malformationen waren.

Zudem verdeutlicht die Tabelle aber auch, dass in einigen anderen Zentren während der TAPVCKorrektur bereits eine partielle cavopulmonale Anastomose hergestellt wurde. Das lässt vermuten, dass bereits vorher ein erster palliativer Schritt unternommen wurde (keine genaueren Angaben in den betreffenden Studien) angeführt und dass dort keine Dringlichkeit zur frühzeitigen TAPVCKorrektur bestand. Im Gegensatz dazu wurde am DHZ immer als erstes die TAPVC korrigiert.

\begin{tabular}{|c|c|c|c|}
\hline & TAPVC-Korrektur & Zusätzliche Korrektur & Genaue Modifikation \\
\hline \multirow[t]{6}{*}{ Hörer et al. } & 193 & 15 & 7 BT-Shunt \\
\hline & & & 4 VSD-Verschluss \\
\hline & & & 2 PA-Banding \\
\hline & & & $1 \mathrm{mBT}$-Shunt \\
\hline & & & 1 Rastelli \\
\hline & & & 1 Klappenplastik \\
\hline \multirow[t]{5}{*}{ Caldarone et al. } & 170 & 37 & 16 BT-Shunt \\
\hline & & & 7 PA-Banding \\
\hline & & & 2 CoA-Plastik \\
\hline & & & 1 Klappen-OP (Aorta) \\
\hline & & & 1 VSD-Verschluss \\
\hline \multirow[t]{2}{*}{ Hancock et al. } & 123 (49 komplex) & 29 & 16 BT-Sunt \\
\hline & & & $2 \mathrm{PCPC}$ \\
\hline \multirow[t]{6}{*}{ Husain et al. } & 51 & $13(25,5 \%)$ & 4 BT-Shunt \\
\hline & & & 3 PA-Banding \\
\hline & & & 2 Glenn (=PCPC) \\
\hline & & & 2 Trikuspidalklappen-OP \\
\hline & & & 1 VSD-Verschluss \\
\hline & & & 1 Aortenbogen-Erweiterung \\
\hline \multirow[t]{4}{*}{ Morales et al. } & 122 & 45 & 4 PA-Banding \\
\hline & & & 14 BT-Shunt \\
\hline & & & 11 Glenn (=PCPC) \\
\hline & & & 16 „andere“ \\
\hline
\end{tabular}

Tabelle 25 Übersicht der durchgeführten initialen Operationen komplexer TAPVC an anderen Zentren

\subsubsection{Alter bei Korrektur}

Die Frage nach dem optimalen Operationsalter haben sich viele Autoren gestellt, denn unterschiedliche Studien belegen einen Zusammenhang zwischen Alter bei Korrekturoperation und Überlebenswahrscheinlichkeit. Auch in der hier vorliegenden Studie wurden die Daten auf diesen Zusammen- 
hang hin analysiert, wobei allerdings keine Korrelation zwischen Alter bei Operation (jünger als 30 Tage versus älter als 30 Tage bei Operation) und Letalitätsraten (weder intra- noch postoperativ) gefunden wurde.

Auch Turley et al. (Turley et al., 1980) stellte schon 1980 fest, dass das Alter bei Operation kein entscheidender Einflussfaktor auf die Überlebenswahrscheinlichkeit ist. Deshalb plädierte er dafür, allein den klinischen Zustand des Patienten als Determinante für den Operationszeitpunkt zu verwenden.

Im Gegensatz dazu fanden Karamlou et al. (Karamlou et al., 2007), Jang et al. (Jang et al., 2010) und Seale et al. (Seale et al., 2010) in ihren Studien, dass jüngeres Alter bei Operation mit einer erhöhten Sterblichkeit assoziiert ist. Es wird von diesen Autoren diskutiert, ob Operationen im frühen Lebensalter vorliegenden pulmonalvenösen Obstruktionen geschuldet sind, welche wegen des kritischen Zustandes die Operation dringlich machen und allein schon aufgrund dessen eine schlechtere Prognose haben. Damit wäre das Operationsalter als solches eventuell nur stellvertretend für eine kritische präoperative Situation zu werten und sollte nicht als unabhängiger Risikofaktor für geringere Überlebenswahrscheinlichkeit gesehen werden (Karamlou et al., 2007).

Schon vor Karamlou et al. zeigte auch Wukasch et al., dass Operationen zur TAPVC-Korrektur im Neugeborenenalter mit einer erhöhten Sterblichkeit assoziiert waren. Er empfiehlt, soweit es der klinische Zustand des Patienten erlaubt, ein Hinauszögern der Operation bis zum sechsten Lebensmonat (Wukasch et al., 1975). Auch viele andere Autoren seiner Zeit erzielten schlechtere Ergebnisse bei jungen Patienten (Cooley, Hallman, \& Leachman, 1966; Gomes et al., 1970; Katz, Kirklin, \& Pacifico, 1978; Mazzucco et al., 1983). Zu beachten ist allerdings, dass die hier genannten Publikationen aus einer Zeit stammen, in der das peri- und postoperative Management Neugeborener mit komplexen kardialen Anomalien sich auf weniger Erfahrung stützte als heute.

Später plädiert Hyde et al. für eine möglichst frühe Operation, vor allem bei Patienten mit präoperativ bestehender pulmonalvenösen Obstruktion (Hyde et al., 1999).

Im DHZ strebt man eine Entscheidung basierend auf dem klinischen Zustand des Patienten an und fällt die Entscheidung individuell auf den Patienten bezogen. Bei ausgeprägter Klinik wird unverzüglich operiert.

Patienten, die erst im Erwachsenenalter korrigierend operiert werden, gibt es aufgrund des schlechten Verlaufs ohne Korrektur nur sehr wenige (Ashoush et al., 1993). Die wenigen erwachsenen Patienten in unserer Kohorte präsentierten sich symptomarm und wurden dennoch korrigierend operiert. Denn in der Literatur wird die Korrektur selbst bei älteren Patienten empfohlen und geht nicht mit einem erhöhten Letalitätsrisiko einher (Berg, Jamieson, \& Pollock, 1986; Shimokawa et al., 1986). Der älteste Patient, der korrigierend operiert wurde und in der Literatur beschrieben wird, war 61 Jahre alt (McManus, Luetzeler, \& Roberts, 1982).

Auch in der hier vorliegenden Studie wurde der Einfluss des Alters bei Korrektur-OP auf Sterblichkeit und Re-Operationswahrscheinlichkeit untersucht. Dabei zeigte sich, dass junges Alter bei Korrektur zwar nicht die Letalität beeinflusst, jedoch das Risiko für die Notwendigkeit einer Re-OP erhöht - sowohl bei der Analyse der gesamten Kohorte, als auch bei der Analyse der Subpopulation der Patienten, die eine isolierte TAPVC aufwiesen. Diese Erkenntnis wird im Kapitel „Risikofaktoren“ genau erörtert. 


\subsubsection{Operations-Details}

Ein häufig diskutierter Punkt ist die Frage nach der Art des kardiopulmonalen Bypasses. Auch wenn einige Autoren (Kirshbom et al., 2002; Lincoln et al., 1988) Operationen unter tiefer Hypothermie nicht als Risikofaktor für erhöhte Sterblichkeit sehen, zeigt sich im Allgemeinen doch ein deutlicher Trend weg von Operationen bei totalem Kreislaufstillstand und hin zu low-flow-Bypass bei moderater Hypothermie, allein schon wegen Bedenken zu neurologischen Schäden durch den Kreislaufstillstand.

Wie auch Boger et al. und Mueller et al. (Boger et al., 1999; Mueller et al., 2013), zeigte auch die hier vorliegende Studie, dass Patienten mit isolierter TAPVC, die bei totalem Kreislaufstillstand operiert wurden eine geringere Überlebenswahrscheinlichkeit hatten, weshalb auch bei unserem Patientenkollektiv seit 2003 die Technik der tiefen Hypothermie komplett verlassen wurde. Genaueres dazu wird anschließend im Kapitel „Risikofaktoren“ besprochen.

In rezenteren Studien taucht auch die Frage der bevorzugten Nahttechnik immer wieder auf. Weil eines der zentralen Probleme nach Korrektur das Auftreten von pulmonalvenösen Stenosen ist, ist das Bestreben vieler Chirurgen die Prävention einer solchen Komplikation. Nachdem das Anwenden einer sutureless-Technik bei Re-Operationen (siehe unten) gute Erfolge zeigt, gibt es von manchen Autoren (Caldarone et al., 1998a; Mueller et al., 2013) mittlerweile die Empfehlung zur suturelessTechnik schon bei initialer Operation. Mueller et al. führt als weiteren Vorteil dieser Technik eine Verkürzung der zur Korrektur nötigen Kreislaufstillstandszeit. Selbige Autoren veröffentlichten vielversprechende erste Ergebnisse bei Korrekturoperationen mit sutureless-Technik. Zum Langzeitverlauf dieser sieben Patienten, eine Re-Stenosierung betreffend, kann allerdings aufgrund der kurzen Nachbeobachtungszeit noch keine Aussage getroffen werden.

Yoshimura et al. schränken die Empfehlung ein. Initiale Operationen unter Anwendung der sutureless-Technik soll nur Risikopatienten vorbehalten sein. Dazu zählen sie Patienten mit HeterotaxieSyndrom, Patienten mit infrakardialem und gemischtem Konnektionstyp und Patienten mit besonders kleinen Gefäßen (Yoshimura et al., 2012).

\subsubsection{Nahtmaterial}

Wie schon oben angedeutet, ist auch die Frage nach dem geeigneten Nahtmaterial eine häufig diskutierte. Dabei handelt es sich um die Naht, die zur Konnektion des pulmonalvenösen Sinus an den linken Vorhof verwendet werden soll, und deren fraglichen Einfluss auf mögliche postoperative Stenosierung an der Anastomose und vielleicht sogar auf Letalität.

Hawkins et al. zeigte einen Vorteil - sowohl Letalität als auch Stenoseraten betreffend - bei der Verwendung von resorbierbarem Nahtmaterial gegenüber nicht resorbierbarem Nahtmaterial. Es wird davon ausgegangen, dass eine durchgehende nicht-resorbierbare Naht das Heilen und Wachsen der Anastomose, vor allem in einem Niederdrucksystem, behindert und sich dadurch postoperativ eine Obstruktion entwickelt (Hawkins et al., 1995). Wilson et al. beschreibt eine durchgehende Naht zur Anastomosierung als einzigen signifikanten Risiko für das Entstehen einer postoperativen pulmonalvenösen Stenose (W. R. Wilson, Jr. et al., 1992). 
Die hier vorgelegte Untersuchung zeigte zwar auch eine schwache Korrelation zwischen nichtresorbierbarem Nahtmaterial und Letalität, allerdings wurde keine statistische Relevanz erreicht $(p=$ 0,079 ). Interessanterweise bestand bei unserem Patientengut keine Korrelation zwischen Art des Nahmaterials und dem Auftreten postoperativer Stenosen. Allerdings ist die Zahl der Patienten die aufgrund einer postoperativen Stenose reoperiert werden mussten, insbesondere derer, bei denen initial nicht-resorbierbares Nahtmaterial verwendet wurde, sehr gering, was die statistische Aussagekraft einschränkt.

\subsubsection{Operative Letalität}

Die intraoperative Letalität war in der gesamten Kohorte sehr gering. Die drei von 193 Patienten, die die Korrekturoperation nicht überlebten, hatten alle eine isolierte TAPVC ohne zusätzliche kardiale Anomalien. Nur bei einem bestand präoperativ eine pulmonale Obstruktion. Aufgrund dieser geringen Zahl an verstorbenen Patienten lässt sich keine relevante Aussage zu Risikofaktoren treffen.

Die operative Letalität wird in rezenteren Studien zwischen 0\% und 15\% angegeben, womit unsere Kohorte im unteren Bereich liegt (Caldarone et al., 1998b; Husain et al., 2012).

Wie schon oben erwähnt haben sich durch Neuerung der Operationstechniken die Ergebnisse der Korrekturoperation seit den 1960er Jahren drastisch gebessert und die Letalitätsraten sind stark gesunken. Ein Trend, der an unserem Institut nicht beobachtet werden konnte. Alle drei verstorbenen Patienten wurden in den 1990er Jahren operiert. Nachfolgend gab es an unserem Institut keine weiteren intraoperativen Letalitäten.

\subsection{Follow up}

Obwohl man sich einig ist, dass die operative und fachgerechte Korrektur einer TAPVC, vor allem wenn sie isoliert auftritt, hohe Überlebensraten ermöglich, bleibt die Nachsorge der operierten Patienten ein wichtiger Faktor, der über die Überlebenswahrscheinlichkeit bestimmt. Dabei gilt es, die Hauptkomplikation, nämlich die pulmonalvenöse Obstruktion möglichst früh zu erkennen und zu behandeln. Nachdem diese, sowie auch andere Komplikationen noch Jahre nach initialer Korrektur auftreten können, ist eine möglichst lückenlose und lange Nachkontrolle essentiell.

Vergleicht man die hier vorgelegte Untersuchung mit anderen Studien ähnlichen Umfangs (Caldarone et al., 1998b; Husain et al., 2012; Karamlou et al., 2007; Lacour-Gayet et al., 1999), so ist dies die Studie mit der längsten Nachbeobachtungszeit.

\subsubsection{Re-Operationen}

Die Frage nach Gründen und Risikofaktoren für die Notwendigkeit einer Re-Operation ist von besonderem Interesse. Nachdem in dieser Studie nicht nur Patienten mit isolierter TAPVC sondern auch Patienten mit zusätzlichen kardialen Fehlbildungen inkludiert sind, sind die Gründe für weitere Operationen am Herzen vielfältig. 
Die häufigste Ursache für Re-Operationen nach TAPVC-Korrektur ist eine sich postoperativ entwickelnde pulmonalvenöse Obstruktion und wird daher ausführlicher besprochen.

\subsubsection{Re-Operation TAPVC}

Postoperative pulmonalvenöse Obstruktion wird in $5 \%$ bis $18 \%$ der Operierten beobachtet (Caldarone et al., 1998a; Hancock Friesen et al., 2005; Karamlou et al., 2007; Lacour-Gayet et al., 1999; Seale et al., 2013) und tritt meist innerhalb der ersten sechs Monate nach Korrektur auf.

Die Pathogenese ist nicht ganz geklärt. Man geht von zwei unterschiedlichen Mechanismen aus. Zum einen kann die Anastomose zwischen Lungenvenen und linkem Atrium bereits initial zu klein sein; Man spricht von einer extrinsischen bzw. anatomischen Obstruktion (Breckenridge, De Leval, Stark, \& Waterston, 1973; Katz et al., 1978; Schafers, Luhmer, \& Oelert, 1987).

Zum anderen wird eine intrinsische Obstruktion beschrieben, die durch Veränderungen der Lungengefäßwand selbst zustande kommt. Intimahyperplasie und Mediahypertrophie sollen eine maßgebliche Rolle spielen (Lacour-Gayet et al., 1999). Diese Gruppe geht mit einer besonders schlechten Prognose einher (Hyde et al., 1999).

Außerdem zeigten Caldarone et al., dass früheres Auftreten mit einer schnelleren Progression der Obstruktion und einer geringerer Überlebenswahrscheinlichkeit vergesellschaftet ist (Caldarone et al., 1998a). Auch andere Autoren zeigten, dass Patienten, die innerhalb der ersten sechs Monate nach der Korrektur eine Obstruktion entwickelten, schwerere Symptome aufwiesen, als die, die erst später vorstellig wurden (Ricci et al., 2003; Seale et al., 2013).

Risikofaktoren, die zur Entwicklung einer solchen pulmonalvenösen Obstruktion führen, werden im Kapitel Risikofaktoren besprochen.

\section{Re-OP Technik}

Die Meinungen zur idealen Technik der Re-Operation bei pulmonalvenöser Obstruktion sind verschieden. In der hier beschriebenen Kohorte wurden unterschiedliche Techniken angewandt. Am häufigsten erfolgte die Erweiterung mittels Patch, so wie sie von Lacour-Gayet et al. empfohlen wird (Lacour-Gayet, 2006). Sonst wurde die Stenose per Inzision erweitert und erneut mit dem Atrium anastomosiert. Hyde et al. empfehlen eine Kombination aus offener Operation und interventioneller Ballondilatation. Wir konnten dafür keinen Vorteil belegen.

Ähnlich wie in unserem Kollektiv, wurden in der Studie von Seale et al. viele unterschiedliche Techniken angewandt, wobei die Wahl jeweils beim Operateur lag. Die Vielfalt und die jeweils geringe Fallzahl der Operationstechniken macht eine genauere Analyse selbst in größeren Kohorten schwierig (Seale et al., 2013).

Eine seltenere und viel diskutierte Methode für die Re-Operation (Devaney, Chang, Ohye, \& Bove, 2006; Najm, Caldarone, Smallhorn, \& Coles, 1998) ist eine Erweiterung mittels sutureless-Technik, die in unserer Kohorte nur einmal zu finden ist.

Diese relativ neue Methode wird von den meisten Autoren als besonders effektiv angesehen (Caldarone et al., 1998a; Yun et al., 2005); denn die Restenosierung soll dadurch am effizientesten verhindert werden (Mueller et al., 2013). Außerdem finden sich sehr niedrige Letalitätsraten (Lacour- 
Gayet, 2006). Mueller et al schlägt deshalb diese Technik auch zur initialen Korrektur der Lungenvenenfehlmündung vor (Mueller et al., 2013). Leider fehlen Langzeitergebnisse dieser Therapiemethode, weshalb trotz erstmaliger Veröffentlichung 1996 (Lacour-Gayet, Rey, \& Planche, 1996) bis jetzt nur wenige Operateure diese Technik anwenden.

Das Anwenden unterschiedlicher Operationstechniken bei der allgemein niedrigen Fallzahl an reoperationsbedürftigen Patienten und unterschiedlich lange Nachbeobachtungszeiten könnte das starke Schwanken der Letalitätsangaben erklären. In der Literatur finden sich Angaben zur Sterblich zwischen $0 \%$ und 64\% (Hyde et al., 1999; Mueller et al., 2013). Mit einer Letalitätsrate von $27 \%$ kommt die Studie von Lacour-Gayet et al. der unseren am nächsten $(16,1 \%)$.

Neben der relativ hohen Letalität, werden auch bei anderen Untersuchungen häufig Rezidivstenosen beobachtet. Auch hier scheint das Anwenden der sutureless-Technik die Restenoseraten deutlich zu verringern (Caldarone et al., 1998a; Gaynor et al., 1999; Ricci et al., 2003).

\subsubsection{Andere kardiale Re-Operationen}

Nachdem diese Studie Patienten mit zusätzlichen kardialen Fehlbildungen inkludiert, mussten oftmals weitere Operation zur Korrektur dieser Anomalien durchgeführt werden. Dabei war der Verschluss eines ASD in diesem Kollektiv am häufigsten. Zur Palliation eines univentrikulären Herzens wird die Vollendung der Fontan-Zikulation angestrebt, wozu mehrere Operationsschritte in unterschiedlichem Alter nötig sind. Die Herstellung des ersten Schrittes wurde in den meisten Fällen bereits im Rahmen der TAPVC-Korrektur (siehe oben) durchgeführt. Die Komplettierung der FontanZirkulation gelang nur bei einem von elf unserer TAPVC-Patienten mit univentrikuärem Herz. Caldarone et al. führte bei 16 Patienten die Anlage eines mBT-Shunts durch; nur bei dreien dieser Gruppe konnte eine TCPC durchgeführt werden.

Im Kapitel „Risikofaktoren“ (6.6) wird das Thema der Kombination aus TAPVC und univentrikulärem Herz mit ihren Problemen ausführlicher diskutiert.

\subsubsection{Letalität}

\subsubsection{Gesamtletalität}

Die gesamte Letalität betrug in dieser Studie 16,1\% und beschreibt alle Todesfälle (intraoperativ und postoperativ) der gesamten Studienkohorte (isolierte TAPVC und kombinierte Vitien). Nachdem auch in der Literatur eine starke die Letalität betreffende Diskrepanz zwischen den zwei Gruppen - Patienten mit isolierter TAPVC und Patienten mit komplexen Anomalien - beschrieben wird, soll auch im hier untersuchten Kollektiv die Letalitätsraten dieser zwei Gruppen gesondert betrachtet werden. Auch in dieser Studie war der Unterschied zwischen den Letalitätsraten der zwei Untergruppen hoch (11,8\% für isolierte TAPVC-Patienten und $62,5 \%$ bei Patienten mit zusätzlichen Anomalien. Dieser Gegensatz wird auch von anderen Autoren so geschildert (Caldarone et al., 1998b; Jang et al., 2010; Michielon et al., 2002).

Was die Gesamtmletalität betrifft, ist ein direkter Vergleich mit anderen Publikationen oft schwierig; 
erstens, aufgrund der Tatsache dass die meisten Studien Patienten mit assoziierten Fehlbildungen ausschließen und somit die Letalitätsrate nicht durch die schlechte Prognose der Patienten mit zusätzlichen Anomalien beeinflusst wird. Und zweitens weil viele Autoren (Cobanoglu \& Menashe, 1993; Lupinetti et al., 1993; Oelert et al., 1986; Sano et al., 1989) eine starke Senkung der Letalität über die letzten Jahre (bzw. im Verlauf der jeweiligen Studie) verzeichneten, was von inhomogenen Letalitätsraten innerhalb der einzelnen Studien zeugt. Diese (von uns nicht beobachtete) chronologische Änderung betrifft wiederum aber nur Patienten, die eine isolierte TAPVC aufweisen, nicht solche, die assoziierte Fehlbildungen haben. Trotz des gleichen prä- und postoperativen Managements für beide Gruppen, bleibt die Letalität der komplexen Fälle über die Jahre unverändert hoch.

Eine Vermutung könnte sein, dass Patienten mit komplexen Fehlbildungen schon vor der Operation bzw. vor der Geburt in einer schlechteren Ausgangssituation befinden. Leider war aus den vorliegenden Akten nicht ersichtlich, ob Patienten im vorliegenden Kollektiv mit komplexen Herzfehlern z.B. Notfallpatienten waren, und sich bereits vor Krankenhausaufnahme in einem schlechteren Zustand befanden.

Ganz allgemein sollte man bei der Betrachtung und bei dem Vergleich unterschiedlicher Studienergebnisse den Follow-up-Zeitraum und die Größe des Patientenkollektives beachten. Je länger das Follow-up und je größer das Patientenkollektiv, desto aussagekräftiger sind gefundene Ergebnisse. Dies gilt auch für Letalitätswahrscheinlichkeiten, womit die vorliegende Studie im Vergleich mit anderen Studien durch ihren langen Nachbeobachtungszeitraum (Caldarone et al., 1998b; Karamlou et al., 2007; Kirshbom et al., 2002) am aussagekräftigsten ist. Denn wenn man Diagramm 9 der Spätletalitäten (Kapitel Ergebnisse) betrachtet fällt auf, dass sich nahezu alle Todesfälle innerhalb der ersten zehn Jahre nach Korrektur ereigneten. Wenn das Follow-up allerdings nur 2 oder 5 Jahre erfasst hätte, dann wäre die Letalitätsrate niedriger, denn einige Patienten wären noch nicht gestorben. Das zeigt, dass Letalitätsangaben nur im Kontext mit der Ausführlichkeit und Länge des Follow-up betrachtet werden sollen. Mit einer durchschnittlichen Nachbeobachtungszeit von 15 Jahren überragt die hier vorgelegte Untersuchung alle anderen bis dato zitierten Studien. Für eine eindeutige Vergeichbarkeit müsste man die exakte Nachbeobachtungszeit von mind. 90\% der Studienpatienten zwischen den Studien vergleichen, diese ist allerdings in den zitierten Studien nicht angegeben.

\subsubsection{Letalität bei isolierter TAPVC}

Bei den bis jetzt besprochenen Letalitätsangaben handelt es sich um GesamtLetalitäten, die Letalitätsraten derer mit isolierter TAPVC und derer mit zusätzlichen Anomalien gemeinsam betrachtend. Viel interessanter ist jedoch eine Aufschlüsselung zwischen Patienten mit isolierter TAPVC und Patienten mit komplexeren Vitien und deren Früh- und Spätletalität.

\subsection{Frühletalität bei isolierter TAPVC}

Die Frühletalität inkludiert alle Patienten, die während des der initialen Operation folgenden Krankenhausaufenthaltes verstorben sind. In diesem Kollektiv verstarben insgesamt mehr als die Hälfte aller verstorbenen Patienten während des ersten Krankenhausaufenthaltes. Damit fällt der Frühletalität ein großer Anteil an der Gesamtletalität zu. Tabelle 26 zeigt Frühletalitätsraten anderer Autoren, die ebenso dem oben beschriebenen zeitlichen Trend folgen. Dies zeigt, dass die oben beschriebene, 
über die Jahre verzeichnete Verbesserung der Gesamtletalität vor allem durch Senkung der Frühletalität bedingt ist.

\begin{tabular}{lll}
\hline Studie & OP-Zeitraum & Frühe Letalität nach OP \\
\hline Bove et al. & $1971-1979$ & $36 \%$ \\
Katz et al. & $1974-1977$ & $21 \%$ \\
Lamb et al. & $1968-1985$ & $18 \%$ \\
Lincoln et al. & $1973-1986$ & $14 \%$ \\
Bando et al. & $1966-1995$ & $10 \%$ \\
Caldarone et al. & $1982-1996$ & $15 \%$ \\
Hyde et al. & $1988-1998$ & $7 \%$ \\
Michielon et al. & $1983-2001$ & $12 \%$ \\
Hier vorliegende Studie & $1974-2011$ & $7,3 \%$ \\
\hline
\end{tabular}

Tabelle 27 Auflistung der Letalitätsraten in Zusammenhang mit dem OP-Jahr

\subsection{Spätletalität bei isolierter TAPVC}

Die Verbesserung der Überlebenschancen für Patienten mit isolierter TAPVC nach Korrektur wird in der Literatur verschiedenen Faktoren zugeschrieben. Eine Rolle spielt die zunehmend akkuratere Diagnostik durch verbesserte echokardiografische Untersuchungsmöglichkeiten (Smallhorn et al., 1987); Verbesserungen der präoperativen Behandlung der Patienten und besseres postoperatives Management, vor allem eine bessere Kontrolle von postoperative pulmonale Hypertension (Bando et al., 1996). Des Weiteren werden Verbesserung der Operationstechniken (W. R. Wilson, Jr. et al., 1992) und zunehmende Erfahrung in der Behandlung besonders junger Patienten verantwortlich gemacht (Bando et al., 1996; Lupinetti et al., 1993; Raisher et al., 1992).

Eine relevante Änderung der Letalität seit 1974 konnte in unserer Studie für keine Gruppe festgestellt werden, was die schlechten Ergebnisse aus den frühen Jahren der Kinderherzchirurgie betrachtend (z.B. 67\% (Mustard \& Dolan, 1957) und 67,6\% (Behrendt et al., 1972)), durchaus überraschend ist.

\begin{tabular}{|c|c|c|c|c|}
\hline & $\begin{array}{l}\text { Gesamtletalität } \\
\text { (Anzahl Patienten) }\end{array}$ & $\begin{array}{l}\text { Nur TAPVC } \\
\text { (Anzahl Patienten) }\end{array}$ & $\begin{array}{l}\text { Komplexere Vitien } \\
\text { (Anzahl Patienten) }\end{array}$ & $\begin{array}{l}\text { Durschnittl. } \\
\text { FU-Zeit }\end{array}$ \\
\hline Vorliegende Studie & $16,1 \%(n=193)$ & $11,8 \%(n=16)$ & $62,5 \%(n=177)$ & 15 Jahre \\
\hline $\begin{array}{l}\text { Caldarone } \\
\text { (Caldarone et al., 1998b) }\end{array}$ & $\sim 25,8 \%(n=170)$ & $26-8 \%(n=126)$ & $52 \%(n=44)$ & 4,7 Jahre \\
\hline $\begin{array}{l}\text { Jang } \\
\text { (Jang et al., 2010) }\end{array}$ & $<10 \%(n=53)$ & & $>40(n=36)$ & 4 Jahre \\
\hline $\begin{array}{l}\text { Lacour-Gayet } \\
\text { (Lacour-Gayet, 2006) }\end{array}$ & $31 \%(n=178)$ & & (nur ein Patient) & 2,1 Jahre \\
\hline $\begin{array}{l}\text { Karamlou } \\
\text { (Karamlou et al., 2007) }\end{array}$ & $\begin{array}{l}40 \text { bzw. } 31,2 \\
(n=377 \text { bzw. } 327)^{*}\end{array}$ & $31,2 \%$ & & 5,2 Jahre \\
\hline
\end{tabular}


Kirshbom

$14 \%(19-5 \%)$

(Kirshbom et al., 2002)

$(n=100)$

$14 \%(n=100)$

5,9 Jahre

Tabelle 28 Übersicht der Letalitäten in anderen Zentren

*Karamlou: von den 377 inkludierten Patienten wurden nur 327 operativ versorgt, weswegen sich zwei unterschiedliche Letalitätswahrscheinlichkeiten ergeben (von den 50 nicht operativ versorgten Patienten sind 49 verstorben)

Des Weiteren schränkt die oftmals unterschiedliche Zusammensetzung des Patientenkollektivs in unterschiedlichen Studien die Vergleichsmöglichkeit der Letalitätsraten weiter ein. Karamlou et al. beschreibt zum Beispiel ein Kollektiv, das, verglichen mit dem hier vorliegenden Kollektiv, einen deutlich geringeren Prozentsatz an Patienten mit präoperativ vorbestehender Obstruktion aufweist. Nachdem die hier gezeigten Ergebnisse eine präoperative Obstruktion als Risikofaktor für eine höhere Letalität zeigen, ist dadurch die Vergleichbarkeit dieser zwei Studien aufgrund der unterschiedlichen präoperativen Ausgangssituation der Patienten eingeschränkt bzw. könnte Unterschiede der Letalitätsraten erklären.

\subsubsection{Letalitätsraten bei komplexen Anomalien}

Bei Patienten mit komplexen kardialen Anomalien zusätzlich zu einer TAPVC finden sich deutlich höhere Letalitätsraten. Diese werden zwischen 52\% und 82\% angegeben (Caldarone et al., 1998b; Gaynor et al., 1999; Sadiq et al., 1996). Auch die hier vorliegende Studie zeigt mit 62\% Letalitätsraten in diesem Bereich. Auch wenn viele Autoren eine Verbesserung der Überlebenswahrscheinlichkeit über die Jahre verzeichneten (Bando et al., 1996; Cobanoglu \& Menashe, 1993; Lamb et al., 1988), konnte man dies trotz zunehmender chirurgischer als auch peri-, intra- und postoperativen Erfahrungswerten nicht für komplexe TAPVC zeigen (Caldarone et al., 1998b; Gaynor et al., 1999). Einige Autoren schreiben dies der allgemein kritischen präoperativen Situation zu. Sadiq et al. konnten belegen, dass die Überlebenswahrscheinlichkeit für dieses Kollektiv, wenn man es weiter einschränkt auf „Notfall-Operationen“ innerhalb des ersten Monates, weiter absinkt auf 13\% (Sadiq et al., 1996).

Damit bleibt die Kombination aus TAPVC und anderen Fehlbildungen, insbesondere univentrikulären Malformationen, eine der Herzfehlbildungen mit der geringsten Überlebenswahrscheinlichkeit.

Nachdem das zusätzliche Vorhandensein von univentrikulären Fehlbildungen nicht nur in der hier vorliegenden Studie einen signifikanten Risikofaktor für geringere Überlebenswahrscheinlichkeit darstellt, wird dies im Anschluss ausführlicher diskutiert.

\subsection{Risikofaktoren}

\subsubsection{Risikofaktoren für geringere Überlebenswahrscheinlichkeit}

Die hier vorgelegte Untersuchung zeigt ein hohes Langzeitüberleben (über 90\%) nach erfolgreicher TAPVC Korrektur. Es gilt nun, Faktoren zu analysieren, welche die Prognose beeinflussen. 
Oftmals sind diese schwer zu beurteilen, da die verschiedenen Faktoren der präoperativen Situation des Patienten einander beeinflussen, z.B. die Tatsache dass der infrakardiale Konnektionstyp meistens mit einer pulmonalvenösen Obstruktion einhergeht.

Lupinetti (Lupinetti et al., 1993) behauptet, dass präoperative Risikofaktoren keine Vorhersage über die postoperativen Ergebnisse erlauben. Dem widerspricht unsere Datenlage.

\subsubsection{Risikofaktoren der gesamten Kohorte: Single Ventricle}

Im gesamten hier untersuchten Patientenkollektiv zeigte sich lediglich das zusätzliche Vorhandensein einer univentrikulären Malformation als statistisch relevanter Risikofaktor für erhöhte Letalität in der multivariaten Analyse.

Auch in der Literatur bedeutet bei einer TAPVC das zusätzliche Vorhandensein eines univentrikulären Herzens eine Anomalie mit sehr schlechter Prognose (Caldarone et al., 1998b; DeLeon et al., 1987; Hancock Friesen et al., 2005; Hashmi et al., 1998; Heinemann et al., 1994; Sadiq et al., 1996). Ähnlich wie in der Studie von Caldarone et al. (Caldarone et al., 1998b) erreichte auch in unserem Patientenkollektiv nur die Hälfe solcher Patienten das fünfte Lebensjahr. Gaynor et al. (Gaynor et al., 1999) beschreiben sogar nur eine 5-Jahres-Überlebenswahrscheinlichkeit von 19\%. Ihr Patientenkollektiv umfasst 73 Patienten, die zusätzlich zur TAPVC auch nur funktionell einen Ventrikel hatten. Davon wurden 61 Patienten operiert, was die 5-Jahres-Überlebenswahrscheinlichkeit von 19\% auf 23\% anhebt. Dies zeigt, dass eine operative Korrektur zwar die Überlebenswahrscheinlichkeit steigert, sie aber dennoch gering bleibt.

Zusätzlich zu den Problemen, die jede einzelne Anomalie für sich mit sich bringen, gibt es mehrere Theorien, weshalb die Überlebenswahrscheinlichkeit bei diesem Zusammentreffen so gering ist.

Zum einen scheinen sich die Pulmonalgefäße bei Patienten mit univentrikulärem Herz schlechter zu entwickeln; Gaynor et al. fanden in histologischen Präparaten der Pulmonalgefäße vermehrt Muskelzellen in der Tunica Media der Gefäßwand (Gaynor et al., 1999), was zu einer pulmonalvenösen Obstruktion und erhöhtem pulmonalen Widerstand führt. Zum anderen erlaubt der oft erhöhte pulmonalarterielle Widerstand bei diesen Patienten meist keine operative Vollendung der FontanZirkulation (s.o.), und damit keine komplette Trennung der zwei Kreisläufe (Driscoll et al., 1992; Pizarro, Mroczek, Gidding, Murphy, \& Norwood, 2006). Nach Anastomosierung der Lungenvenen mit dem Atrium kann es sein, dass die oft zu kleinen und eventuell strukturell veränderten Gefäße per se, insbesondere aber in Kombination mit dem Ein-Kammer-Herz zu wiederkehrender pulmonaler Obstruktion führen, was zusätzlich die Überlebenswahrscheinlichkeit senkt (Jenkins et al., 1993).

Des Weiteren ist auf Grund des schlechten klinischen Zustandes in den ersten Lebenswochen bei solchen Patienten oftmals eine notfallmäßige Operation notwendig, was ebenfalls zur schlechten Prognose beitragen könnte (Heinemann et al., 1994).

Zwei interessante Studien kommen aus dem Children's Hospital of Philadelphia. Gaynor et al. untersuchten ein relativ großes Patientenkollektiv von insgesamt 73 Patienten mit Single Ventricle und TAPVC. Inkludiert waren alle Patienten mit diesen Diagnosen auch die, die schon vor einer OP verstarben oder die in anderen Zentren voroperiert waren. Die 5-Jahres-Überlebenswahrscheinlichkeit für die Gruppe der operierten Patienten $(n=61)$ lag bei $23 \%$. Initiale Operation war allerdings nur in 31 Fällen eine Korrektur der TAPVC, davon wurde bei 29 Patienten zusätzlich eine erste Palliation der 
univentrikulären Malformation vorgenommen (Aorto-Pulmonaler-Shunt, PCPC, Norwood-OP etc). Überlebensraten unterscheiden sich je nach initialer Intervention. Gemeinsam ist der Gruppe der operierten Patienten aber, dass initiale TAPVC-Korrektur mit schlechterer Überlebenswahrscheinlichkeit assoziiert ist ( $p=0.02)$, am schlechtesten für Patienten mit obstruierter TAPVC.

Ein weiterer, in der Literatur diskutierter Risikofaktor ist junges Alter bei initialer Operation. (Gaynor et al., 1999). Von dieser Studie ausgehend, untersuchten Lodge et al. am selben Institut fünf Jahre später eine weitere Gruppe an Patienten mit gleicher Konstellation der Anomalien ( $n=18)$, die in der Zwischenzeit operiert wurden. Dazu verglichen sie die beiden Gruppen und konnten in der späteren Gruppe eine Überlebenswahrscheinlichkeit von über $50 \%$ feststellen, was eine deutliche Besserung der Operationsergebnisse zeigt. Allerdings sind einige Limitierungen zu beachten. Zum einen sind in der zweiten Gruppe viel weniger Patienten als in der ersten, was die Aussagekraft deutlich beeinträchtigt. Außerdem konnten die Autoren selbst keinen handfesten Unterschied im klinischen Management oder in Operationstechniken ausmachen, welcher verantwortlich gemacht werden könnte für die besseren Ergebnisse. Einzige Neuerung im zweiten Patientenkollektiv waren fünf Herztransplantationen(davon eine Herz-Lungen-Transplantation), wovon zwei die initiale Prozedur waren. Beide Patienten überlebten den Eingriff. Die anderen drei Transplantationen erfolgten nach fehlgeschlagener initialer Korrektur, alle drei wiesen postoperative PVO auf. Noch fehlen Langzeitergebnisse für die Transplantierten (Follow-Up bis jetzt lediglich 13 Monate), die eine Überlegenheit gegenüber der konventionellen Therapie zeigen könnten. Ungeachtet der Probleme des Spendermangels und der lebenslangen Immunsuppression, bleibt eine Herztransplantation für diese Diagnose doch ein bemerkenswerter Ansatz (Lodge et al., 2004). Langzeitergebnisse aus diesen beiden Studien stehen noch aus.

Ein Vergleich dieser Daten mit unserem Kollektiv ist schwer, weil nicht bei allen Patienten die TAPVC korrigiert wurde, sondern hauptsächlich bei denen, die bereits zum Operationszeitpunkt eine Obstruktion manifestierten, was per se mit einer schlechteren Prognose vergesellschaftet ist und auch für eine anschließende Fontan-Zirkulation ein prognostisch ungünstiger Faktor ist (Hosein et al., 2007). Trotzdem bleibt die Erkenntnis, dass isolierte, initiale TAPVC-Korrektur bei kombinierten Vitien das Überleben dieser Patienten nicht verbessert, interessant.

\subsubsection{Risikofaktoren bei isolierter TAPVC: PVO, totaler Kreislaufstillstand}

Um eine Aussage zu den Risikofaktoren für die Letalität für die Gruppe der Patienten mit isolierter TAPVC treffen zu können, führten wir eine multivariate Analyse der selben Faktoren im Kollektiv ohne Patienten mit assoziierten Anomalien durch und erhielten oben angeführte Ergebnisse. Nachdem die Letalitätsrate in der Untergruppe der Patienten mit isolierter TAPVC mittlerweile in allen größeren Zentren sehr niedrig ist, ist eine aussagekräftige Analyse der Risikofaktoren schwierig, da die Anzahl der Verstorbenen sehr gering ist. Das könnte auch erklären, weshalb von verschiedenen Autoren ganz unterschiedliche Risikofaktoren genannt werden. Hier aufgeführt sind nur die nach den eigenen Daten tatsächlich als Risiko erkannten Faktoren. Von anderen Autoren gefundene Risikofaktoren wurden bereits unter den jeweiligen Überschriften diskutiert.

$>$ Pulmonalvenöse Obstruktion (präoperativ) 
Als wichtigsten Risikofaktor fanden wir eine präoperativ bestehende pulmonalvenöse Obstruktion.

Es ist der häufigste auch von anderen Autoren beschriebene Risikofaktor für erhöhte Letalität nach TAPVC-Korrektur (Bando et al., 1996; Boger et al., 1999; Caldarone et al., 1998b; Hancock Friesen et al., 2005; Sinzobahamvya, Arenz, Brecher, Blaschczok, \& Urban, 1996). Es gibt mehrere mögliche Ursachen einer pulmonalvenösen Obstruktion. Am meisten diskutiert wird die Rolle der Mündungsstelle der fehlmündenden Lungenvenen, die durch den Konnektionstyp beschrieben wird. Grundsätzlich können alle Konnektionstypen eine PVO bedingen (Gathman \& Nadas, 1970), am häufigsten wird sie aber bei Mündung in die Vena Portae (infrakardialer Typ) beobachtet (Newfeld, Wilson, Paul, \& Reisch, 1980). Das erklärt, warum, wie in anderen Arbeiten auch, in dieser Studie der größte Anteil der Patienten mit PVO eine infrakardiale Konnektion aufweisen ((Hyde et al., 1999; Karamlou et al., 2007) Anderson). Auch vaskuläre Veränderungen im Lungenstromgebiet tragen zur Erhöhung des pulmonalen Widerstandes bei und aggravieren somit zu einer pulmonalvenösen Obstruktion. Dabei handelt es sich sowohl um Intimahyperplasie als auch um eine Hypertrophie der Muskelzellen der Media, die sich bis in die peripheren Arteriolen und Venolen fortsetzen kann (Haworth \& Reid, 1977). Newfeld et al. zeigen, dass sich diese Veränderungen schon während der ersten Lebenswochen finden und nicht unbedingt mit dem pulmonalen Druck korrelieren, was bedeutet, dass solche vaskulären Veränderungen sich nicht unbedingt als pulmonalvenöse Obstruktion manifestieren müssen, sondern auch bei (noch) asymptomatischen Patienten vorliegen können (Newfeld et al., 1980). Auch Gaynor et al. zeigen an pathologisch auffälligen histologischen Präparaten klinisch unauffälliger Patienten, dass solche Veränderungen schon vor Manifestation einer pulmonalvenösen Obstruktion bestehen können (Gaynor et al., 1999). Haworth et al. geht sogar davon aus, dass diese strukturellen Veränderungen bereits pränatal existieren. In einer histologischen Studie zeigt er, dass pathologische Wandveränderungen bereits in den ersten Lebenstagen auftreten. Diese Gefäßwandveränderungen macht er verantwortlich für die zwar selten auftretenden, dafür schwerwiegenden pulmonalen, hypertensiven Krisen im postoperativen Verlauf. Des Weiteren beschreibt er bei infrakardialer Konnektion der Lungenvenen besonders kleine Gefäße, welche möglicherweise zu erhöhten Raten an postoperativen Stenosen führen könnten (Haworth, 1982). In der hier vorliegenden Studie war infrakardiale Konnektion allerdings kein Risikofaktor für postoperative Stenose der Lungenvenen.

Die Tatsache, dass sich eine präoperativ bestehende PVO auch in unserem Kollektiv postoperativ normalisiert, weist darauf hin, dass diese vaskulären Veränderungen jedoch oft nach Normalisierung der hämodynamischen Situation reversibel sind. Eine Studie von Endo et al., die histologische Veränderungen der Pulmonalgefäße bei Patienten mit angeborener PVO untersucht, unterstützt diese These und fügt dem die Aussage hinzu, dass aufgrund der Reversibilität der histologischen Veränderungen auch Patienten mit PVO selbst im späteren Verlauf noch korrigierend operiert werden können (Endo, Yamaki, Ohmi, \& Tabayashi, 2000).

Auch die Größe des Shunts auf Vorhofebene (Foramen ovale bzw. ASD) könnte eine Rolle spielen (Berman, Fishbone, \& Stansel, 1972). Theoretisch würde ein kleines Shuntvolumen hämodynamisch zu einem erhöhten rechtsatrialen Druck führen, was zu pulmonaler Hypertension und konsekutiv zu einer PVO führen würde. Gathman et al. (Gathman \& Nadas, 1970) 
zeigen aber in ihrer Arbeit keinen signifikanten Zusammenhang zwischen atrialen Druckwerten und pulmonaler Hypertension.

Als Folge der PVO steigt der pulmonalvenöse Druck, in schwerwiegenderen Fällen auch der pulmonalarterielle Druck bis auf Werte, die über dem systemischen Druck liegen. Der pulmonale Blutfluss sinkt. Das führt einerseits zu einer Druckbelastung des rechten Ventrikels und andererseits zu einem Abfall der systemischen arteriellen Sauerstoffsättigung. Zusätzlich nehmen die oben genannten vaskulären Veränderungen der Pulmonalgefäße zu. Als Resultat kommt es zu einer Rechtsherzbelastung (Hypertropie, später Dilatation) und durch den verminderten Sauerstoffgehalt zu Gewebehypoxie und zu metabolischer Azidose. Bove et al. bestätigen das mit ihrer Erkenntnis, dass eine präoperativ bestehende schwere Azidose der wichtigste Risikofaktor für Frühletalität bei Patienten mit infrakardialem Konnektionstyp ist (Bove et al., 1975).

In unserem Patientenkollektiv fällt auf, dass mehr als die Hälfte der bislang verstorbenen Patienten, die präoperativ eine PVO aufwiesen bereits während des ersten Krankenhausaufenthaltes starben. Fast alle starben an Herzinsuffizienz, welche durch eine solche Gewebehypoxie bedingt sein könnte.

Eine weitere Theorie zur Ursache der erhöhten Sterblichkeit der Patienten mit PVO ist die Hypothese, dass bei diesen Patienten die pulmonalen Arteriolen unterentwickelt sind. Maeda et al. (Maeda, Yamaki, Yokota, Murakami, \& Takamoto, 2004) zeigten an histologischen Präparaten, dass die Arteriolen der Patienten mit PVO im Verhältnis zu den begleitenden Bronchiolen einen erheblich kleineren Radius aufwiesen, als die der Patienten ohne PVO. Sie vermuten, dass es dadurch zu einem erhöhten pulmonalen Widerstand kommt, auch wenn die oben beschrieben Wandveränderungen der Gefäße selbst gering sind. In diesem Falle kann es auch nach Korrektur zu persistierender pulmonalen Hypertension kommen, womit aufgrund der bestehen bleibenden Rechtsherzbelastung selbst kleine Stimuli schon zu einer Herzinsuffizienz führen können, was zur FrühLetalität beiträgt.

Auch wenn viele Autoren PVO als Risikofaktor ansehen, gibt es einige, die andere Erkenntnisse gewonnen haben. Serraf et al. gibt an, dass präoperative PVO keinen negativen Einfluss auf die Prognose hat, solange eine pulmonale Hypertension prä-, peri- und postoperativ vermieden werden kann (Serraf et al., 1991), macht aber keine genauere Angaben zum Procedere. Sinzobahamvya et al. erkennen PVO zwar als Risikofaktor für erhöhte Letalität, zeigten aber, dass vor allem Fortschritte im postoperativen Management diesen Risikofaktor neutralisieren konnten (Sinzobahamvya et al., 1996). Auch Wang et al. fanden keine erhöhte Letalität für Patienten mit PVO, allerdings ist die Aussagekraft gering, da in dieser Studie nur die operative Letalität betrachtet wird (P. Y. Wang et al., 2004).

Die hier vorgelegte Untersuchung hat, verglichen mit anderen Arbeiten ((Husain et al., 2012; Hyde et al., 1999; Karamlou et al., 2007)), einen hohen Anteil an Patienten mit präoperativer PVO, was die Aussagekraft dieser Studie verbessert.

\section{$\underline{\text { Totaler Kreislaufstillstand }}$}

Seit des Einführung des totalen Kreislaufstillstandes in tiefer Hypothermie in den 1960er Jahren kam es zu einer deutlich geringeren operativen Letalitätsrate und war lange Zeit Standard 
in vielen auf Kinderherzchirurgie spezialisierten Zentren (Barratt-Boyes et al., 1971; Kirklin, Dawson, Devloo, \& Theye, 1961; Weiss et al., 1960). Auch am Deutschen Herzzentrum wurde bis in die 1990er Jahre diese Methode bei der Korrektur dieses Vitiums gewählt. Denn Vorteile sind ein blutleeres OP-Feld ohne störende Kanülen der Herzlungenmaschine und damit bessere Sicht auf das sehr kleine OP-Gebiet. Durch die Erkenntnis, dass bei tiefer Hypothermie die Ischämietoleranz der Organe verlängert ist (Gravlee, 2000), wusste man, dass für die Zeit des totalen Kreislaufstillstandes die Organe geschützt sind. Das Organ mit der kürzesten Ischämietoleranz ist das Gehirn. Die Zuverlässigkeit der Hirnprotektion hängt u.a. von der Qualität der Kühlung ab und ist zeitlich limitiert. Berichte über - trotz sorgfältigster Bemühungen - anschließende zerebrale Dysfunktionen und spätneurologische Folgen ließen Zweifel an dieser Methode aufkommen (Molina et al., 1984; Newburger et al., 1993; Treasure et al., 1983).

Die möglichen ischämischen Folgeschäden werden in der Literatur lebhaft diskutiert. Einige Studien zeigten eine Abnahme des Intelligenzquotienten und Entwicklungsverzögerungen abhängig von der Dauer des totalen Kreislaufstillstandes (Settergren et al., 1982; Wells, Coghill, Caplan, \& Lincoln, 1983; Wright, Hicks, \& Newman, 1979). Andere Studien konnten diese Folgen nicht belegen, solange die Zeit des totalen Kreislaufstillstandes unter 60 Minuten beträgt bei einer Temperatur von $20^{\circ} \mathrm{C}$ (Clarkson, MacArthur, Barratt-Boyes, Whitlock, \& Neutze, 1980; Dickinson \& Sambrooks, 1979; Messmer, Schallberger, Gattiker, \& Senning, 1976). Grundsätzlich ist es schwierig mögliche Folgeschäden adäquat zu interpretieren, wenn der Vergleich mit einer Kontrollgruppe fehlt. Zusätzlich gilt es zu beachten, dass Kinder mit angeborenen Herzfehlern auch noch andere Ursachen aufweisen können für eine verzögerte Entwicklung bzw. geistige Retardierung. Neurologische Dysfunktion kann selbst nach einer extrakorperalen Zirkulation ohne DHCA oder nach einer Operation ohne Herz-LungenMaschine auftreten. Somit ist es schwierig die Auswirkungen eines totalen Kreislaufstillstandes unabhängig zu beurteilen (Berthrong \& Sabiston, 1951; Clark \& Tyler, 1957; Cottrill \& Kaplan, 1973; Linde, Rasof, \& Dunn, 1967; Linderkamp et al., 1979; Terplan, 1973).

Man ist sich einig, dass die Länge der Zeit des totalen Kreislaufstillstandes mit einem höheren Risiko für Folgeschäden korreliert. Viele haben versucht eine "sichere“ Zeitdauer festzulegen, eine definitive Aussage konnte noch nicht getroffen werden. Diese variiert zwischen 30 bis 45 Minuten (Newburger et al., 1993; Richard F. Davis, 2008), manche erachten eine Kreislaufstillstandzeit von bis zu 60 Minuten noch als "sicher" (Clarkson et al., 1980; Dickinson \& Sambrooks, 1979).

Neben Langzeitfolgen, wie IQ-Minderung und Entwicklungsverzögerungen, kann es auch zu kurzfristigen Folgen kommen. Postoperative Krampfanfälle werden bei ca. 20\% der Neugeborenen nach Operationen mit totalem Kreislaufstillstand beobachtet. Subklinische Anfälle, welche lediglich im EEG verzeichnet sind, sind noch häufiger zu beobachten (Coles et al., 1984; Tharion et al., 1982). Dies ist besonders zu beachten, wenn Neugeborene postoperativ noch relaxiert sind und deswegen keine motorischen Entäußerungen bieten können. Meist sind die Anfälle selbstlimitierend und treten postoperativ auch bei Kindern auf, welche nicht unter totalem Kreislaufstillstand operiert wurden. 
Die Langzeitprognose ist unklar. Einige sehen keine Langzeitschäden, andere Autoren beschreiben pyschomotorische Vernänderungen zusätzlich zu neurologischen Auffälligkeiten, einhergehend mit MRT-nachweisbaren Parenchymläsionen (Rappaport et al., 1998).

Eine weitere neurologische Manifestation eventualer Schäden nach totalem Kreislaufstillstand ist die Choreoathetose, eine neurologische Störung, welche durch Hyperkinesien vor allem der Arme und Beine, aber auch der Gesichtsmuskulatur gekennzeichnet ist. Dies wird bei 1 bis 20\% der Kinder, welche bei totalem Kreislaufstillstand operiert wurden, beobachtet (Brunberg, Doty, \& Reilly, 1974; Clarkson et al., 1980). Dies tritt meist innerhalb der ersten 2 bis 6 postoperativen Tage auf und nimmt an Ausprägung im zeitlichen Verlauf ab. Nur bei sehr schweren Fällen bleiben choreatiforme Bewegungsstörungen oder eine generalisierte Hypotonie bestehen (Brunberg, Reilly, \& Doty, 1974).

Als Alternative zum totalen Kreislaufstillstand wird eine low-flow-Technik angewandt, bei der bei moderater Hypothermie ein reduzierter Blutfluss belassen wird. Eine bessere Kardioprotektion wurde außerdem gezeigt (Buckberg, 1979; Ellis et al., 1980). Rebeyka et al. zeigten, dass die kortikalen Funktionen erhalten bleiben, aber die Energieressourcen in Form von Phosphaten reduziert wird, was auch dem low-flow-Bypass ein zeitliches Limit aufzwingt (Rebeyka et al., 1987). Sie zeigten, dass auch während reduzierten Flussraten die kortikale Funktion (gemessen anhand von somatosensorischen Potenzialen) erhalten bleibt, was bei totalem Kreislaufstillstand nicht der Fall ist (Coles et al., 1984). Swain et al. fanden ähnliche Ergebnisse; zusätzlich zeigten sie, dass der Phosphatverbrauch abhängig ist von der Flussrate: je höher die Flussrate, desto geringer der Energieverbrauch, desto längere die Reserve (Swain et al., 1991). Nachteil der Methode ist, dass eine Mindestflussrate, welche als "sicher" angesehen wird, nicht definiert ist. Newburger et al. (Newburger et al., 1993) zeigten, dass zwar ihrer Meinung nach low-flow-Bypass eine protektive Wirkung auf Gehirn und Herz hat, führten aber als Nachteil der Methode eine längere Bypasszeit an, welche wiederum einhergeht mit erhöhtem Risiko für Komplikationen der extrakorporalen Zirkulation. Dazu zählen insbesondere Embolien, Hämolyse und Gerinnungsstörungen und Entzündungsaktivierung (Greeley \& Ungerleider, 1991; Hammeke \& Hastings, 1988; Javid et al., 1969; Johnston et al., 1991; Lee, Brady, Rowe, \& Miller, 1971; Sotaniemi, 1983; Sotaniemi, Juolasmaa, \& Hokkanen, 1981).

Weitere Einflüsse und Komplikationen, sowohl des totalen Kreislaufstillstandes als auch des Low-flow-Bypasses werden in der Literatur diskutiert. Tassani et at. beschrieb z.B. eine geringere systemische Entzündungsreaktion bei Patienten, die im totalen Kreislaufstillstand operiert wurden.

Die hier vorliegende Studie war allerdings nicht darauf ausgelegt, internistische oder neurologische Spätschäden bzw. „nicht-chirurgische“-Komplikationen zu beurteilen. Daten zur neurologischen bzw. psychomotrischen Entwicklung der Patienten z.B. waren nicht erhoben worden. 
Zusammenfassend ist zu sagen, dass die postoperativ unterschiedlichen Auswirkungen der zwei verschiedenen Methoden vielfältig sind. Auch wenn die hier vorgelegte Studie ein schlechteres Überleben bei Korrekturoperation im totalen Kreislaufstillstand belegt (wie auch viele andere), gilt es für den Operateur, technische Gegebenheiten zu berücksichtigen. Bei totalem Kreislaufstillstand ist das Herz komplett blutleer und somit die Sicht auch das gesamte Operationsfeld uneingeschränkt. Auch bedarf es keiner "störenden“ Kanülen im Operationsfeld. Bei einem Low-flow-Bypass bleibt ein reduzierter Blutfluss bestehen, was ggf. die Sicht beeinträchtigen kann. Sollte es dadurch zu vermehrten chirurgischen postoperativen Komplikationen kommen, müssen etwaige technische Begebenheiten gegenüber der Bypassmethode abgewogen werden und eine individuelle Risiken-Nutzen-Abwägen bedacht werden. Bezüglich Flussrate, Körpertemperatur, und weiteren Parametern der Perfusion entscheidet der Chirurg im Einzelfall.

Am Deutschen Herzzentrum München änderte sich mit dem geänderten Bypassprozedere auch die Operationstechnik. Strebte man früher (vor 1990) den intrakardialen Zugang zur Anastomosierung an, erfolgte dieser nach 1990 meist von extrakardial. Dadurch ist eine gute Sicht trotz Blutfluss intrakardial an der Stelle der Anastomose gegeben. Eine Ära-spezifische Letalitätsänderung konnte in der hier vorliegenden Studie allerdings nicht gezeigt werden, womit beide Methoden als gleichwertig anzusehen sind.

\subsubsection{Risikofaktoren für Notwendigkeit einer Re-OP}

Eine weitere Frage dieser Studie war die Frage nach Risikofaktoren für die Notwendigkeit einer späteren Re-Operation. Nachdem ein häufig beobachtetes Problem der TAPVC-Korrektur die Stenosierung der Anastomose und damit einer Obstruktion der Lungenvenen ist, ist eine Differenzierung der Art der Re-Operation nach ihrer Ursache sinnvoll:

- Re-Operation zur Behebung einer Obstruktion (Re-OP-TAPVC)

Hierbei geht es um Operationen, die bei Patienten notwendig wurden, welche an der Stelle der neuen Konnektion der Lungenvenen mit dem linken Atrium eine Stenose entwickelt haben.

- Kardiale Re-Operation aus anderer Indikation

\subsubsection{Risikofaktor Re-OP TAPVC: Alter $<30$ Tage}

Einzig relevanter Risikofaktor für erhöhte Re-Operationswahrscheinlichkeit ist nach den hier vorgelegten Ergebnissen ein geringes ( $<30$ Tage) Alter bei Korrektur. Dies gilt sowohl für die Patienten mit isolierter TAPVC als auch für diejenigen mit kardialen Begleitfehlbildungen. Dabei ist anzumerken, dass nach diesen Daten geringes Alter bei Korrektur keinen Einfluss auf die Sterblichkeit nimmt. Das erlaubt die Schlussfolgerung, dass die Überlebenswahrscheinlichkeit nicht von der Notwendigkeit einer Re-Operation abhängig ist und Patienten, die wegen postoperativ entstandener Pulmonalvenenstenose reoperiert werden müssen, keine geringere Überlebenswahrscheinlichkeit aufweisen. Hancock-Friesen et al. zeigt im Gegensatz dazu, dass die Notwendigkeit einer Re-Operation die Über- 
lebenswahrscheinlichkeit senkt (Hancock Friesen et al., 2005). Allerdings ist zu beachten, dass die Anzahl der reoperierten Patienten sowohl in dem hier vorliegenden Kollektiv, als auch in den genannten Studien sehr gering ist. Noch geringer ist die Anzahl derer, die nach der Re-Intervention verstorben sind. Somit ist die Aussagekraft bei geringer Fallzahl statistisch gesehen schwach, sodass hieraus keine Handlungsempfehlung abgeleitet werden kann. bestätigend zeigten aber auch andere Studien eine Korrelation zwischen jungem Patientenalter bei Korrektur und erhöhtem Risiko für ReOperationsbedarf. Ähnliche Ergebnisse lieferten die Studien von Karamlou, Oelert und Caldarone (Caldarone et al., 1998a; Karamlou et al., 2007; Oelert et al., 1986)

Die Frage nach Risikofaktoren für Re-Operationen beschäftigte auch andere Autoren und erbrachte unterschiedliche Ergebnisse. So zeigten Husain et al. (Husain et al., 2012), dass lange Aortenklemmund lange Bypasszeiten, sowie perioperativ bestehende pulmonalvenöse Obstruktion das Risiko für eine Re-Operation erhöhen. Obwohl in unserer Analyse diese Faktoren im Gegensatz dazu keine unabhängigen Risikofaktoren darstellten, lässt sich doch ein hypothetischer Vergleich aufstellen. Die von uns isolierten Hauptrisikofaktoren, die gesamte Kohorte betreffend (also auch jene mit zusätzlichen Anomalien), nämlich SV-Malformation und präoperativ bestehende PVO, könnten sich auch in den von Husain et al. beschriebenen Faktoren widerspiegeln, wenn man bedenkt, dass sich die längsten Operationszeiten in unserer Studie bei Operationen fanden, die nicht nur die TAPVC korrigieren sondern auch erste operative Schritte zur Palliation der SV-Physiologie unternahmen. Dies zeigt deutlich, dass längere Operationszeiten nur als Surrogatparameter zu sehen sind und die eigentlichen Risikofaktoren die Komplexität der Anatomie und der damit verbundenen Korrektur, sowie die Qualität der chirurgischen Technik sind. Auch kann man davon ausgehen, dass bereits perioperativ bestehender erhöhter pulmonalvenöser Druck längerfristig mit den Schwierigkeiten einer pulmonalvenösen Obstruktion einhergehen wird.

Bando et al. zeigten, dass die Größe der Pulmonalvenen eine signifikante Determinante sowohl für Sterblichkeit, als auch für postoperativ entstehende Pulmonalvenenstenose ist (Bando et al., 1996). Nachdem die anatomische Größe der Pulmonalvenen auch die präoperative Situation beeinflusst und schon vor Korrektur zu einer pulmonalvenösen Obstruktion führen kann, ist sie als unabhängige Parameter schwer zu beurteilen. Denn die selbe Publikation zeigte auch eine Korrelation zwischen präoperativer pulmonaler Obstruktion und Sterblichkeit. Allerdings ergab auch eine große multizentrische Studie, dass kleine Größen pulmonaler Gefäße signifikant mit erhöhter Sterblichkeit und postoperativer PVO assoziiert sind. Aufgrund der großen Fallzahl $(n=406)$ ist diese Aussagekraft dieser Studie hoch (Seale et al., 2013).

Ein anderer diskutierter Risikofaktor ist die Wahl des Nahtmaterials für die Anastomose. Unter anderem Hyde et al. sahen weniger Stenose-bedingte Re-Operationen bei Anastomosierung von Lungenvenen und linkem Atrium mit resorbierbarem Nahtmaterial. Die Hypothese hierzu ist, dass durch nicht resorbierbares Nahtmaterial das Wachstum der Gefäße an der Stelle der Anastomose behindert ist, und es somit im Verlauf zur relativen Stenosierung kommt (Hawkins et al., 1995; Hyde et al., 1999). Einen signifikanten Zusammenhang zwischen Art des Nahtmaterials und erhöhtem Re-OPBedarf zeigte die Analyse der hier vorliegenden Daten jedoch nicht. Allenfalls konnte eine Trend zwischen der Verwendung von nicht resorbierbarem Nahtmaterial und höherer Letalität erkannt werden, der aber keine statistische Signifikanz auf dem 5\% Niveau erreichte. Bei genauerer Analyse fällt allerdings auf, dass von den Patienten, bei denen nicht-resorbierbares Nahtmaterial verwendet wurde, sechs der insgesamt acht Verstorbenen während des ersten Krankenhausaufenthaltes verstarben. Todesursachen waren unterschiedlich, aber aufgrund der kurzen Zeitspanne zwischen Korrektur 
und Todeszeitpunkt ist die Entwicklung postoperativer Stenosen durch Nahtmaterial-bedingte Wachstumshemmung unwahrscheinlich.

Damit ist die Verwendung nicht resorbierbarer Nähte zwar nach den hier vorgelegten Daten mit einer höheren Sterblichkeit assoziiert, nicht aber mit einer höheren Stenose-Rate.

Auch der Einfluss des anatomischen Konnektionstyps auf die Entwicklung postoperativer Stenosen wird diskutiert. So zeigt die Studie von Karmalou et al. ein höheres Risiko für postoperative Stenosen bei Patienten mit gemischtem Lungenvenenkonnektionstyp. Auch Caldarone et al. konnten Patienten mit infra- und kardialem Konnektionstyp ein erhöhtes Re-Operationsrisiko zuweisen (Caldarone et al., 1998a; Karamlou et al., 2007). Ein Zusammenhang zwischen Re-Stenoserate und anatomischer TAPVC-Mündung konnte in der hier vorgelegten Studie nicht gezeigt werden.

Des Weiteren ist die Frage nach dem Einfluss unterschiedlicher Operationstechniken auf die Wahrscheinlichkeit von Re-Stenosen naheliegend. Wie oben erwähnt (Mueller et al.), behaupten Wilson et al., dass selbst eine geringe venöse Obstruktion am Ende der Operation zu anschließender Fibrosierung und damit fortschreitenden Stenosierung führen kann (W. R. Wilson, Jr. et al., 1992). Schon vor dieser Publikation zeigte die Arbeit von Smallhorn et al., dass echokardiographisch feststellbare Turbulenzen an der Anastomose der Lungenvenen das Risiko für Stenosen an dieser Stelle erhöhen (Smallhorn et al., 1987). Caldarone et al. schließt, dass operative "Imperfektionen“ an der Anastomose durch Nahtmaterial, Nahtverlauf und Gewebsverletzung der Lungengefäße zu Turbulenzen des Blutflusses führen, was in der Kombination mit der lokalen Verletzung durch die Naht zu einer Stenosierung führen können. Deswegen postuliert er eine Minimierung des operativen Traumas, zum Beispiel durch Suture-less-Technik (Caldarone et al., 1998a).

Mueller et al. führten daraufhin zwischen 2009 und 2012 bei sieben Patienten eine Primärkorrektur mit oben genannter sutureless-Technik durch. Drei dieser Patienten präsentierten präoperativ eine PVO, die anderen vier nicht. Alle sieben Patienten lebten am Ende der Nachbeobachtung beschwerdefrei, ohne postoperative Stenosierung. Allerdings ist der Nachbeobachtungszeitraum sehr kurz $(1,8$ Jahre) und erlaubt noch keine Aussage zum Langzeitverlauf. Auch die Fallzahl ist noch zu gering, um einen soliden Vergleich zur herkömmlichen Operationstechnik zu erlauben.

\subsubsection{Risikofaktoren für kardiale Re-Operationen: univentrikuläre Malformation}

Neben der oben besprochenen Gruppe der Re-Operationen zur Behebung einer nach TAPVCKorrektur entstandenen pulmonalvenösen Obstruktion, wurde hier eine Analyse zur Erhebung der Risikofaktoren für kardiale Re-Operationen auch anderer Art durchgeführt. Die Gründe für kardiale Re-Operationen sind vielfältig, und ihr Vorkommen im Allgemeinen selten. Es ist somit schwierig, statistisch relevante Aussagen über diese kleine, heterogene Gruppe zu treffen. Auch in der Literatur limitiert sich die Frage nach kardialen Re-Operationen im Allgemeinen auf Re-Operationen zur Korrektur postoperativ auftretender Stenosen, die bereits oben diskutiert wurden. Re-Operationen anderer kardialer Genese werden kaum beschrieben, nicht zuletzt, weil diese selten und von unterschiedlichster Art sind. Dabei handelt es sich meist um weitere Schritte einer bereits begonnenen Palliation assoziierter Herzfehler (Gaynor et al., 1999; Husain et al., 2012).

Als Risikofaktor für Re-OP konnte hier nur das Vorliegen einer univentrikulären Malformation nachgewiesen werden. Der naheliegende Grund hierfür ist, dass zur Palliation der univentrikulären Mal- 
formation ohnehin mehrere operative Schritte notwendig sind. Der erste Schritt wurde dabei meist gleichzeitig im Rahmen der TAPVC-Korrektur durchgeführt. Nachdem diese palliativen Maßnahmen für ein Überleben notwendig sind, ist verständlich, dass trotz der geringen Fallzahl statistische Relevanz erreicht wurde. Die einzigen zwei Patienten unserer Kohorte von Patienten mit TAPVC und SVMalformation, die zu am Ende der Datenerhebung noch lebten, waren ein Patient mit erfolgreich hergestellter Fontan-Zirkulation, und ein Patient mit PCPC vor Fontan-Komplettierung.

Der Vergleich mit anderen Publikationen ist für diese Gruppe nicht einfach. Zum einen aufgrund der Tatsache, dass andere Autoren die Versorgung der Single-Ventrikel-Physiologie als initiale Prozedur beschreiben, womit die TAPVC-Korrektur die Re-Operation darstellen würde. Zum anderen korrigierten einige die TAPVC bei manchen Patienten mit SV nicht (Hashmi et al., 1998; Razzouk et al., 1995). Nachdem die primäre TAPVC-Korrektur Einschlusskriterium für das hier untersuchte Kollektiv war, ist somit die Vergleichbarkeit mit diesen Publikationen eingeschränkt.

Morales et al. beschreibt eine Kollektiv von insgesamt 122 Patienten, von denen 38 zusätzlich univentrikuläre Malformationen aufwiesen. Davon konnte bei insgesamt 8 Patienten eine FontanKomplettierung erreicht werden. Alle diese Patienten lebten zum am Ende der Datenerhebung noch (Morales et al., 2006) . Allerdings betrug die durchschnittliche Follow-up Zeit nur fünf Jahre. Bei diesem besonderen Patientenkollektiv ist die Sterblichkeit naturgemäß umso höher, je länger die Nachbeobachtungszeit ist. Damit könnte die längere Nachbeobachtungszeit auch ein erklärender Faktor für die erhöhte Letalität der hier untersuchten Kohorte sein.

Andere Autoren beschreiben univentrikuläre Malformationen mit TAPVC als eigenen Risikofaktor für postoperative pulmonalvenöse Stenosen und dafür notwendige Re-Operationen (Gaynor et al., 1999; Morales et al., 2006).

Die Analyse der hier vorliegenden Daten ergab als einzigen relevanten Risikofaktor (bezogen auf die gesamte Studienkohorte) für die Notwendigkeit einer kardialen Re-OP die zusätzliche Diagnose eines univentrikulären Herzens. Dies verwundert nicht, da die Komplettierung der Fontanzirkulation in meheren Operationen erfolgt. Für die Untergruppe der Patienten mit isolierter TAPVC ließ sich kein statistisch signifikanter Risikofaktor isolieren. 


\section{Schlussfolgerung}

Zielsetzung dieser Arbeit war die Identifizierung potentieller Risikofaktoren einerseits für die Letalität und andererseits für die Notwendigkeit kardialer Re-Operationen bei Patienten mit TAPVC. Nachdem bereits in der Literatur beschrieben wird, dass Patienten mit TAPVC und zusätzlichen kardialen Anomalien eine bedeutend schlechtere Prognose aufweisen, ermittelten wir die Risikofaktoren für die Endpunkte Überleben und Freiheit von Re-OPs nicht nur für die gesamte Kohorte, sondern auch gesondert für die Patienten mit isolierter TAPVC und die Patienten mit zusätzlich Anomalien.

Die Überlebenswahrscheinlichkeit für operierte Patienten mit isolierter TAPVC ist sehr gut, wohingegen die Überlebenswahrscheinlichkeit für Patienten mit zusätzlichen, insbesondere univentrikulären, Malformationen gering ist. Diese Ergebnisse zeigten sich am DHZ auch über die letzten Dekaden als unverändert.

Als Risikofaktor für erhöhte Letalität bei Patienten mit zusätzlichen Malformationen zeigte sich das Vorhandensein univentrikulärer Malformationen als prognostisch schlechten Einflussfaktor auf das Überleben. Trotz verbesserter peri- und postoperativer Maßnahmen fand sich über die letzten Dekaden keine relevante Prognoseverbesserung für Patienten mit univentrikulären Malformationen.

Bei der Gruppe der Patienten mit isolierter TAPVC zeigten sich zwei Risikofaktoren für erhöhte Letalität. Zum einen das Vorhandensein präoperativer pulmonalvenöser Obstruktion und zum anderen eine Korrektur bei totalem Kreislaufstillstand.

Bereits 2003 ist man am DHZ davon abgekommen, bei totalem Kreislaufstillstand zu operieren, und ging dazu über, die TAPVC unter low-flow-Bypass zu korrigieren. Die hier erhobenen Daten legen nahe dass dieser Strategiewechsel in der Erfahrung des DHM von Vorteil ist .

Re-Operationen bei Patienten mit isolierter TAPVC waren selten nötig. Bei Patienten mit zusätzlichen Malformationen deutlich öfter, naturgemäß aufgrund der oft mehrzeitigen Behandlung der anderen Fehlbildungen. Verständlich ist somit die Erkenntnis, dass das Vorhandensein univentrikulärer Malformationen als einziger Risikofaktor für Re-Operationen identifiziert wurde und es nach Datenlage auch keine Möglichkeit gibt das Risiko dieser Patienten zu senken.

Für Patienten mit isolierter TAPVC zeigte sich ein junges Patientalter bei Korrektur als einziger Risikofaktor für anschließende Re-Operation. Grund für die Notwendigkeit einer neuerlichen Operation war eine postoperativ entstandene Obstruktion im Bereich der anastomosierten Lungenvenen. Nachdem das Patientenalter mit vielen anderen Faktoren korreliert (siehe Diskussion) lässt sich daraus jedoch nicht die Empfehlung ableiten, Patienten so spät wie möglich zu operieren. Denn nachdem allein die klinische Präsentation des Patienten das Entscheidungskriterium für den Operationszeitpunkt ist, kann davon ausgegangen werden, dass die Patienten, welche bereits früh operiert wurden, durch eine präoperative pulmonalvenöse Obstruktion bereits kritisch krank waren.

\section{Offene Forschungsfragen}

Nachdem dies eine retrospektive Studie ist, waren die Einflussmöglichkeiten auf die auf die Qualität der Daten bei einem so langen Zeitraum gering. Dies trifft auch auf nahezu alle anderen in dieser 
Arbeit zitierten Arbeiten zu. Die Rarität der untersuchten Anomalie, Unterschiede und Entwicklungen im prä, peri- und postoperativem Management und kurze Nachbeobachtungszeiten in der Literatur machen die Entwicklung und Umsetzung neuer chirurgischer Strategien schwierig.

Auch wenn die Langzeitergebnisse bei operierten TAPVC-Patienten - wie hier gezeigt werden konnte -befriedigend sind, gibt es eindeutig Risikofaktoren, die die Prognose negativ beeinflussen.

In der Zusammenschau der Literatur bleiben heute folgende Fragen offen:

In wie weit beeinflusst die Größe des ASD bzw. Foramen Ovale die präoperative Obstruktion?

(Ein Vergleich der Sonographie- und Katheterbefunde der jeweiligen Gruppen könnte dazu einen Überblick verschaffen. Allerdings ist die Konsequenz dieser Erkenntnis fraglich, denn es wird vermutet, dass die durch die Obstruktion entstehenden Gefäßwandveränderungen bereits pränatal beginnen, womit selbst eine Vergrößerung des ASD direkt postnatal wenig Effekt hätte. Außerdem wird mittlerweile eine möglichst frühe Korrektur angestrebt, die nicht unbedingt durch zusätzliche präoperative Maßnahmen verzögert werden sollte.

Kann durch die „sutureless“ Technik eine postoperative Obstruktion verhindern werden?

Zur Klärung dieser Frage wäre eine prospektiv randomisierte Studie ideal. Aufgrund der niedrigen Prävalenz postoperativ entstehender Obstruktion selbst schon bei herkömmlicher Technik, würde eine große Fallzahl benötigt um statistisch signifikante Ergebnisse zu erhalten. Solche Fallzahlen könnten durch ein multizentrisches Studiendesign erreicht werden, was aber wiederum andere Limitierungen mit sich bringt. Eine solche Studie wird derzeit von der European Congenital Heart Surgeons Association durchgeführt. Die Ergebnisse sind zum Zeitpunkt der Abgabe der Studie noch nicht publiziert.

Schlechte Prognose bei univentrikulärem Herzen

Die TAPVC mit Obstruktion und univentrikulärem Herzen ist auch heute noch ein Herzfehler, bei dem das Erreichen des Erwachsenenalter nahezu unmöglich ist. Die Betretung eines solchen Kindes stellt für die Familie eine große Belastung dar. Weiterführende psychologische Studien sind nötig um das Ausmaß dieser Belastung und der Folgen für die Eltern und mögliche Geschwister zu beurteilen. Auf der Basis dieser Daten können individuelle Gesprächen mit den Eltern auch bereits pränatal objektiver geführt werden. 


\section{Zusammenfassung}

Grundlagen: Bei der totalen Lungenvenenfehlmündung handelt es sich um einen seltenen angeborenen Herzfehler, welcher isoliert, oder in Kombination mit weiteren Anomalien (insbesondere mit univentrikulären Malformationen) auftritt und durch das zusätzliche Vorhandensein einer pulmonalvenösen Obstruktion verkompliziert werden kann.

Ziel dieser Studie ist es, mögliche Risikofaktoren für Spätletalität und Notwendigkeit einer ReOperation zu identifizieren.

Methoden: Hierzu wurden demographische, prä-, peri- und postoperative Daten aller Patienten, bei denen zwischen 1974 und 2011 am Deutschen Herzzentrum München eine TAPVC korrigiert wurde, gesammelt und mittels multivariater Cox-Regression analysiert. Die durchschnittliche, Nachbeobachtungszeit betrug $15,0 \pm 11,0$ Jahre.

Ergebnis: Die Überlebensrate für das gesamte Kollektiv betrug 82,7 $\pm 2,9 \% 20$ Jahre nach initialer Korrektur. Relevanter Risikofaktor für Letalität in der multivariaten Analyse war das zusätzliche Vorhandensein einer univentrikulären Malformation (5,9\%, $p<0,001)$. In der Gruppe der Patienten mit isolierter TAPVC ( $n=177)$, ergaben sich in der univariaten Analyse eine präoperativ bestehende pulmonalvenöse Obstruktion $(53,7 \% \mathrm{p}<0,030)$ und die Durchführung der Korrektur-OP unter komplettem Kreislaufstillstand $(78,5 \% \mathrm{p}<0,017)$ als Risikofaktoren für Letalität. Die Freiheit von kardialen ReOperationen betrug 20 Jahre nach initialer Korrektur $82,2 \pm 3,3 \%$. Der einzige relevante Risikofaktor für die Notwendigkeit einer neuerlichen Operation in der multivariaten Analyse ist das zusätzliche Vorhandensein einer univentrikulären Malformation $(p<0,001)$. Die Freiheit von Reoperationen aufgrund postoperativer pulmonalvenöser Obstruktion betrug 90,4 $\pm 2,5 \%$. Als Risikofaktor hierfür zeigte sich in der univariaten Analyse das Patientenalter $\leq 30$ Tage bei initialer Korrekturoperation, sowohl für die gesamte Kohorte $(p<0,007)$, als auch für die Untergruppe der Patienten mit isolierter TAPVC $(p<0,022)$.

Schlussfolgerung: Das reoperationsfreie Überleben der Patienten mit isolierter TAPVC ist bis ins 3. Lebensjahrzehnt exzellent und nicht von der Anatomie der TAPVC abhängig. Im Gegensatz dazu zählt die Überlebenswahrscheinlichkeit von Patienten mit TAPVC und zusätzlichen univentrikulären Malformationen zu den Schlechtesten aller angeborenen Herzfehler. Postoperative Pulmonalvenenstenosen sind selten und treten vor allem bei Patienten auf, die als Neugeborene operiert wurden 


\section{Abbildungsverzeichnis}

1Wahrscheinlichkeitsdarstellung für Freiheit von Re-OPs nach Kaplan Meier...... 31

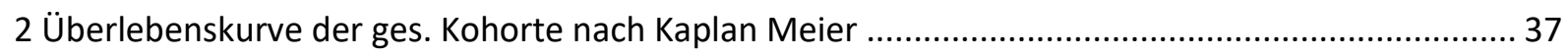

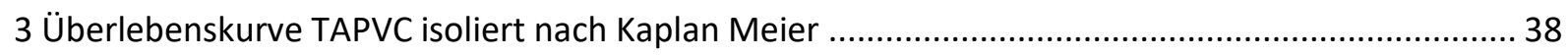

4 Überlebenskurve Vergleich präop. pulm. Hypertonie ja/nein .......................................................... 38

5 Überlebenskurve nach Kaplan Meier Vergleich isoliert/komplex................................................... 39

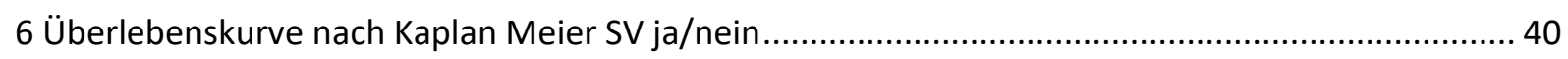

7 Überlebenskurve nach Kaplan Meier Vergleich Heterotaxie ja/nein............................................. 40

9 Kaplan-Meier - Überlebenskurve für Patienten 10 Kaplan-Meier - Überlebenskurve für Patienten 43 


\section{Tabellenverzeichnis}

Tabelle 1 Häufigkeitsverteilung der einzelnen TAPVC Typen im Patientenkollektiv ........................... 23

Tabelle 2 Anzahl der Patienten mit PVO und Aufschlüsselung der jeweiligen TAPVC-Typen............... 24

Tabelle 3 Anteil an Patienten mit PVO je nach TAPVC-Typ.............................................................. 24

Tabelle 4 Assoziierte kardiale Fehlbildungen bei Patienten mit TAPVC.............................................. 25

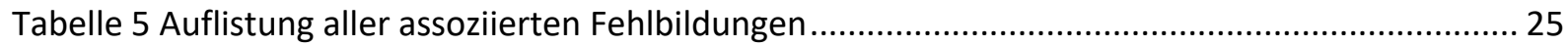

Tabelle 6 Liste der im Rahmen der Korrektur-Operation zusätzlich durchgeführter chirurgischer

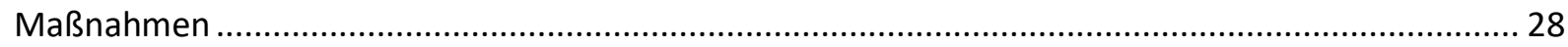

Tabelle 7 intraoperative Letalität der Korrektur-OP …….................................................................... 29

Tabelle 8 Liste aller frühen Todesfälle mit Todesursachen .................................................................. 30

Tabelle 9 Anteil an Re-Operationen in der gesamten Kohorte …..................................................... 31

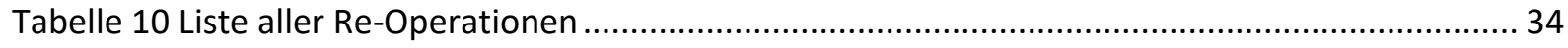

Tabelle 11 Liste aller Re-Operationen und Letalitätsraten .............................................................. 35

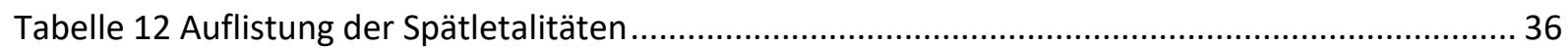

Tabelle 13 Überblick der Überlebenswahrscheinlichkeiten............................................................... 37

Tabelle 14 Übersicht Überlebenswahrscheinlichkeiten der verschiedenen Untergruppen .................. 37

Tabelle 15 Aufschlüsselung der Patienten mit komplexen Fehlbildungen und Anzahl der

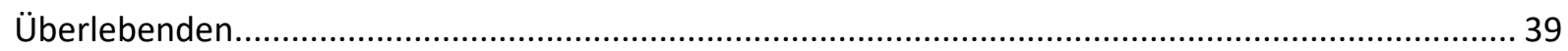

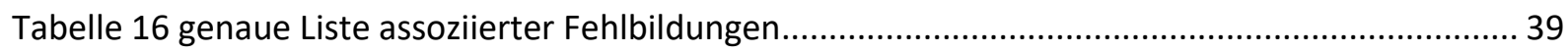

Tabelle 17 Ergebnisse der univariaten Testung für erhöhte Letalität (gesamte Kohorte).................... 42

Tabelle 18 Ergebnisse der multivariaten Testung für erhöhte Letalität (gesamte Kohorte) ................. 42

Tabelle 19 Ergebnisse der univariaten Testung für erhöhte Letalität (Patienten mit isolierter TAPVC)

Tabelle 20 Übersicht relevanter Risikofaktoren für Letalität

Tabelle 21 Übersicht der Ergebnisse der Analyse der gesamten Kohorte auf Notwendigkeit von TAPVC- Re-OPs

Tabelle 22 Übersicht der Ergebnisse der Analyse der Untergruppe an Patienten mit isolierter TAPVC auf Notwendigkeit von TAPVC- Re-OPs.

Tabelle 23 Übersicht der Ergebnisse der Analyse der gesamten Kohorte auf Notwendigkeit für andere kardiale Re-OPs . .47

Tabelle 24 Übersicht Risikofaktoren für Re-Operationen

Tabelle 25 Übersicht der durchgeführten initialen Operationen komplexer TAPVC an anderen Zentren 


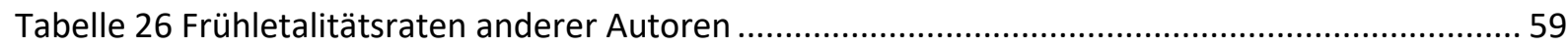

Tabelle 27 Auflistung der Letalitätsraten in Zusammenhang mit dem OP-Jahr .................................. 60

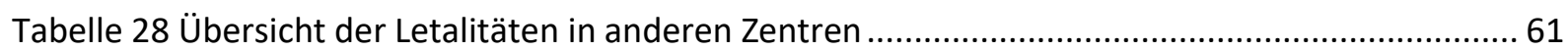




\section{Diagrammverzeichnis}

Diagramm 1 Patienten mit und ohne präoperativer PVO

Diagramm 2 TAPVC-Typen bei Patienten mit Obstruktion ......................................................... 24

Diagramm 3 Aufschlüsselung Alter bei Operation.............................................................. 21

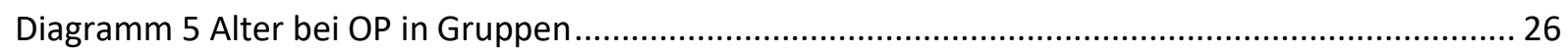

Diagramm 6 Altersverteilung zum Zeitpunkt der Korrektur-Operation........................................ 27

Diagramm 9 : Häufigkeitsverteilung des Patientenalters zum Zeitpunkt der Korrektur-Operation ..... 27

Diagramm 10 Anzahl der Korrektur-OPs bei totalem Kreislaufstillstand bzw bei Low-flow-Zirkulation

Diagramm 11 Anteil der lebenden und verstorbenen Patienten, geteilt nach Untergruppen TAPVC isoliert und komlexe Vitien. 36

Diagramm 13 Spätletalitäten 59 


\section{Abkürzungsverzeichnis}

OP

BSA

TAPVC

VSD

TGA

MAG

PDA

ASD

AVSD

PFO

SV

PCPC

TCPC

BT-Shunt

mBT-Shunt

$\mathrm{HLH}$

DHZ

FU

PVO

AP-Shunt

RV

DORV

LV

DOLV

PS

TI

GI-Blutung

DHCA
Operation

body surface area (Körperoberfläche)

total anomalous pulmonary venous connection

Ventrikelseptumdefekt

Transposition großer Arterien

Malposition großer Arterien

persistierender Ductus arteriosus

Atriumseptumdefekt

atrioventrikulärer Septumdefekt

persistierendes Foramen ovale

single ventricle

partial cardio-pulmonary connection

total cardio-pulmonary connection

Blalock-Taussig-Shunt

modifizierter Blalock-Taussig-Shunt

hypoplastisches Linksherz

Deutsches Herzzentrum München

Follow-up

pulmonalvenöse Obstruktion

aortopulmonaler Shunt

rechter Ventrikel

Double outlet right ventricle

linker Ventrikel

Double outlet left ventricle

Pulmonalklappenstenose

Trikuspidalklappeninsuffizienz

Gastrointestinale Blutung

deep hypothermic cardiac arrest (Kreislaufstillstand in tiefer Hypothermie) 


\section{Literaturverzeichnis}

Dieses Literaturverzeichnis wurde mit dem Literaturverwaltungsprogramm EndNote erstellt und nach dem Zitationsstil „APA 6th“ formatiert.

Anderson, R. H., MD, Edward J. Baker, MA, MD, FRCP, FRCPCH, Daniel J. Penny, MD, Andrew N. Redington, MD, Michael L. Rigby, MD, and Gil Wernovsky, MD. (2010). Paediatric Cardiology.

Ashoush, R., Jebara, V. A., el Rassi, I., Badawi, G., Sarkis, A., Asmar, B., Checrallah, E. (1993). Total anomalous pulmonary venous connection in adults--a surgical review. J Med Liban, 41(4), 230-235.

Bando, K., Turrentine, M. W., Ensing, G. J., Sun, K., Sharp, T. G., Sekine, Y., Brown, J. W. (1996). Surgical management of total anomalous pulmonary venous connection. Thirty-year trends. Circulation, 94(9 Suppl), II12-16.

Barratt-Boyes, B. G., Simpson, M., \& Neutze, J. M. (1971). Intracardiac surgery in neonates and infants using deep hypothermia with surface cooling and limited cardiopulmonary bypass. Circulation, 43(5 Suppl), 125-30.

Behrendt, D. M., Aberdeen, E., Waterson, D. J., \& Bonham-Carter, R. E. (1972). Total anomalous pulmonary venous drainage in infants. I. Clinical and hemodynamic findings, methods, and results of operation in 37 cases. Circulation, 46(2), 347-356.

Berg, G. A., Jamieson, M. P., \& Pollock, J. C. (1986). Repair of total anomalous pulmonary venous connection in adults. Thorac Cardiovasc Surg, 34(6), 359-361. doi: 10.1055/s-2007-1022173

Berman, M. A., Fishbone, G., \& Stansel, H. C., Jr. (1972). Total anomalous pulmonary venous return with obstruction at the foramen ovale. Successful palliation by open atrial septectomy. Am J Surg, 124(5), 679-681.

Berthrong, M., \& Sabiston, D. C., Jr. (1951). Cerebral lesions in congenital heart disease, a review of autopsies on 162 cases. Bull Johns Hopkins Hosp, 89(5), 384-406.

Bharati, S., \& Lev, M. (1973). Congenital anomalies of the pulmonary veins. Cardiovasc Clin, 5(1), $23-$ 41.

Boger, A. J., Baak, R., Lee, P. C., Boersma, E., Meijboom, F. J., \& Witsenburg, M. (1999). Early results and long-term follow-up after corrective surgery for total anomalous pulmonary venous return. Eur J Cardiothorac Surg, 16(3), 296-299.

Bove, K. E., Geiser, E. A., \& Meyer, R. A. (1975). The left ventricle in anomalous pulmonary venous return. Morphometric analysis of 36 fatal cases in infancy. Arch Pathol, 99(10), 522-528.

Breckenridge, I. M., De Leval, M., Stark, J., \& Waterston, D. J. (1973). Correction of total anomalous pulmonary venous drainage in infancy. J Thorac Cardiovasc Surg, 66(3), 447-453.

Brown, V. E., De Lange, M., Dyar, D. A., Impastato, L. W., \& Shirali, G. S. (1998). Echocardiographic spectrum of supracardiac total anomalous pulmonary venous connection. J Am Soc Echocardiogr, 11(3), 289-293.

Brunberg, J. A., Doty, D. B., \& Reilly, E. L. (1974). Choreoathetosis in infants following cardiac surgery with deep hypothermia and circulatory arrest. J Pediatr, 84(2), 232-235.

Brunberg, J. A., Reilly, E. L., \& Doty, D. B. (1974). Central nervous system consequences in infants of cardiac surgery using deep hypothermia and circulatory arrest. Circulation, 50(2 Suppl), II6068. 
Buckberg, G. D. (1979). A proposed "solution" to the cardioplegic controversy. J Thorac Cardiovasc Surg, 77(6), 803-815.

Burchell, H. B. (1956). Total anomalous pulmonary venous drainage: clinical and physiologic patterns. Proc Staff Meet Mayo Clin, 31(6), 161-167.

Burroughs, J. T., \& Edwards, J. E. (1960). Total anomalous pulmonary venous connection. Am Heart J, 59, 913-931.

Byrum, C. J., Dick, M., Behrendt, D. M., \& Rosenthal, A. (1982). Repair of total anomalous pulmonary venous connection in patients younger than 6 months old. Late postoperative hemodynamic and electrophysiologic status. Circulation, 66(2 Pt 2), 1208-214.

Caldarone, C. A., Najm, H. K., Kadletz, M., Smallhorn, J. F., Freedom, R. M., Williams, W. G., \& Coles, J. G. (1998a). Relentless pulmonary vein stenosis after repair of total anomalous pulmonary venous drainage. Ann Thorac Surg, 66(5), 1514-1520.

Caldarone, C. A., Najm, H. K., Kadletz, M., Smallhorn, J. F., Freedom, R. M., Williams, W. G., \& Coles, J. G. (1998b). Surgical management of total anomalous pulmonary venous drainage: impact of coexisting cardiac anomalies. Ann Thorac Surg, 66(5), 1521-1526.

Carter, R. E., Capriles, M., \& Noe, Y. (1969). Total anomalous pulmonary venous drainage. A clinical and anatomical study of 75 children. Br Heart J, 31(1), 45-51.

Choe, Y. H., Lee, H. J., Kim, H. S., Ko, J. K., Kim, J. E., \& Han, J. J. (1994). MRI of total anomalous pulmonary venous connections. J Comput Assist Tomogr, 18(2), 243-249.

Chungsomprasong, P., Soongswang, J., Nana, A., Durongpisitkul, K., Loahaprasitiporn, D., Vijansorn, C., \& Sriyodchartti, S. (2011). Medium and long-term outcomes of Fontan operation. [Evaluation Studies]. J Med Assoc Thai, 94(3), 323-330.

Clark, D. B., \& Tyler, H. R. (1957). Incidence of neurological complications in congenital heart disease. AMA Arch Neurol Psychiatry, 77(1), 17-22.

Clarkson, P. M., MacArthur, B. A., Barratt-Boyes, B. G., Whitlock, R. M., \& Neutze, J. M. (1980). Developmental progress after cardiac surgery in infancy using hypothermia and circulatory arrest. Circulation, 62(4), 855-861.

Cobanoglu, A., \& Menashe, V. D. (1993). Total anomalous pulmonary venous connection in neonates and young infants: repair in the current era. Ann Thorac Surg, 55(1), 43-48; discussion 48-49.

Coles, J. G., Taylor, M. J., Pearce, J. M., Lowry, N. J., Stewart, D. J., Trusler, G. A., \& Williams, W. G. (1984). Cerebral monitoring of somatosensory evoked potentials during profoundly hypothermic circulatory arrest. Circulation, 70(3 Pt 2), 196-102.

Cooley, D. A., Hallman, G. L., \& Leachman, R. D. (1966). Total anomalous pulmonary venous drainage; correction with the use of cardiopulmonary bypass in 62 cases. J Thorac Cardiovasc Surg, 51(1), 88-102.

Correa-Villasenor, A., Ferencz, C., Boughman, J. A., \& Neill, C. A. (1991). Total anomalous pulmonary venous return: familial and environmental factors. The Baltimore-Washington Infant Study Group. Teratology, 44(4), 415-428. doi: 10.1002/tera.1420440408

Cottrill, C. M., \& Kaplan, S. (1973). Cerebral vascular accidents in cyanotic congenital heart disease. Am J Dis Child, 125(4), 484-487.

Craig, J. M., Darling, R. C., \& Rothney, W. B. (1957). Total pulmonary venous drainage into the right side of the heart; report of 17 autopsied cases not associated with other major cardiovascular anomalies. Lab Invest, 6(1), 44-64. 
de Leval, M. R. (2005). The Fontan circulation: a challenge to William Harvey? Nat Clin Pract Cardiovasc Med, 2(4), 202-208. doi: 10.1038/ncpcardio0157

DeLeon, S. Y., Gidding, S. S., Ilbawi, M. N., Idriss, F. S., Muster, A. J., Cole, R. B., \& Paul, M. H. (1987). Surgical management of infants with complex cardiac anomalies associated with reduced pulmonary blood flow and total anomalous pulmonary venous drainage. Ann Thorac Surg, 43(2), 207-211.

Delisle, G., Ando, M., Calder, A. L., Zuberbuhler, J. R., Rochenmacher, S., Alday, L. E., Van Praagh, R. (1976). Total anomalous pulmonary venous connection: Report of 93 autopsied cases with emphasis on diagnostic and surgical considerations. Am Heart J, 91(1), 99-122.

Delius, R. E., de Leval, M. R., Elliott, M. J., \& Stark, J. (1996). Mixed total pulmonary venous drainage: still a surgical challenge. J Thorac Cardiovasc Surg, 112(6), 1581-1588.

Devaney, E. J., Chang, A. C., Ohye, R. G., \& Bove, E. L. (2006). Management of congenital and acquired pulmonary vein stenosis. Ann Thorac Surg, 81(3), 992-995; discussion 995-996. doi: 10.1016/j.athoracsur.2005.08.020

Devaney, E. J., Ohye, R. G., \& Bove, E. L. (2006). Pulmonary vein stenosis following repair of total anomalous pulmonary venous connection. Semin Thorac Cardiovasc Surg Pediatr Card Surg Annu, 51-55. doi: 10.1053/j.pcsu.2006.02.023

Di Eusanio, G., Sandrasagra, F. A., Donnelly, R. J., \& Hamilton, D. I. (1978). Total anomalous pulmonary venous connection (surgical technique, early and late results). Thorax, 33(3), 275282.

Dickinson, D. F., \& Sambrooks, J. E. (1979). Intellectual performance in children after circulatory arrest with profound hypothermia in infancy. Arch Dis Child, 54(1), 1-6.

Driscoll, D. J., Offord, K. P., Feldt, R. H., Schaff, H. V., Puga, F. J., \& Danielson, G. K. (1992). Five- to fifteen-year follow-up after Fontan operation. Circulation, 85(2), 469-496.

Du Bois, D., \& Du Bois, E. F. (1989). A formula to estimate the approximate surface area if height and weight be known. 1916. Nutrition, 5(5), 303-311; discussion 312-303.

Edwards, J. E. (1953). Pathologic and developmental considerations in anomalous pulmonary venous connection. Proc Staff Meet Mayo Clin, 28(17), 441-452.

Ellis, R. J., Wisniewski, A., Potts, R., Calhoun, C., Loucks, P., \& Wells, M. R. (1980). Reduction of flow rate and arterial pressure at moderate hypothermia does not result in cerebral dysfunction. $J$ Thorac Cardiovasc Surg, 79(2), 173-180.

Endo, M., Yamaki, S., Ohmi, M., \& Tabayashi, K. (2000). Pulmonary vascular changes induced by congenital obstruction of pulmonary venous return. Ann Thorac Surg, 69(1), 193-197.

Friedlich, A., Bing, R. J., \& Blount, S. G., Jr. (1950). Physiological studies in congenital heart disease; circulatory dynamics in the anomalies of venous return to the heart including pulmonary arteriovenous fistula. Bull Johns Hopkins Hosp, 86(1), 20-57.

Gathman, G. E., \& Nadas, A. S. (1970). Total anomalous pulmonary venous connection: clinical and physiologic observations of 75 pediatric patients. Circulation, 42(1), 143-154.

Gaynor, J. W., Collins, M. H., Rychik, J., Gaughan, J. P., \& Spray, T. L. (1999). Long-term outcome of infants with single ventricle and total anomalous pulmonary venous connection. $J$ Thorac Cardiovasc Surg, 117(3), 506-513; discussion 513-504.

Gomes, M. M., Feldt, R. H., McGoon, D. C., \& Danielson, G. K. (1970). Total anomalous pulmonary venous connection. Surgical considerations and results of operation. $J$ Thorac Cardiovasc Surg, 60(1), 116-122. 
Gravlee, G. P. (2000). Cardiopulmonary Bypass: Principles and Practice, 2nd edition (2nd Edition ed.). New York: Churchill Livingstone.

Greeley, W. J., \& Ungerleider, R. M. (1991). Assessing the effect of cardiopulmonary bypass on the brain. Ann Thorac Surg, 52(3), 417-419.

Hammeke, T. A., \& Hastings, J. E. (1988). Neuropsychologic alterations after cardiac operation. J Thorac Cardiovasc Surg, 96(2), 326-331.

Hammon, J. W., Jr., Bender, H. W., Jr., Graham, T. P., Jr., Boucek, R. J., Jr., Smith, C. W., \& Erath, H. G., Jr. (1980). Total anomalous pulmonary venous connection in infancy. Ten years' experience including studies of postoperative ventricular function. J Thorac Cardiovasc Surg, 80(4), 544551.

Hancock Friesen, C. L., Zurakowski, D., Thiagarajan, R. R., Forbess, J. M., del Nido, P. J., Mayer, J. E., \& Jonas, R. A. (2005). Total anomalous pulmonary venous connection: an analysis of current management strategies in a single institution. Ann Thorac Surg, 79(2), 596-606; discussion 596-606. doi: 10.1016/j.athoracsur.2004.07.005

Hashmi, A., Abu-Sulaiman, R., McCrindle, B. W., Smallhorn, J. F., Williams, W. G., \& Freedom, R. M. (1998). Management and outcomes of right atrial isomerism: a 26-year experience. J Am Coll Cardiol, 31(5), 1120-1126.

Hastreiter, A. R., Paul, M. H., Molthan, M. E., \& Miller, R. A. (1962). Total anomalous pulmonary venous connection with severe pulmonary venous obstruction. A clinical entity. Circulation, 25, 916-928.

Hawkins, J. A., Minich, L. L., Tani, L. Y., Ruttenberg, H. D., Sturtevant, J. E., \& McGough, E. C. (1995). Absorbable polydioxanone suture and results in total anomalous pulmonary venous connection. Ann Thorac Surg, 60(1), 55-59.

Haworth, S. G. (1982). Total anomalous pulmonary venous return. Prenatal damage to pulmonary vascular bed and extrapulmonary veins. Br Heart J, 48(6), 513-524.

Haworth, S. G., \& Reid, L. (1977). Structural study of pulmonary circulation and of heart in total anomalous pulmonary venous return in early infancy. Br Heart J, 39(1), 80-92.

Heinemann, M. K., Hanley, F. L., Van Praagh, S., Fenton, K. N., Jonas, R. A., Mayer, J. E., Jr., \& Castaneda, A. R. (1994). Total anomalous pulmonary venous drainage in newborns with visceral heterotaxy. Ann Thorac Surg, 57(1), 88-91.

Hofbeck, M., Koch, A., Buheitel, G., Gerling, S., Rauch, R., Weyand, M., \& Singer, H. (2000). Late postoperative cardiac arrhythmias after total cavopulmonary anastomosis and correlation with age of the patients at operation. $Z$ Kardiol, 89(9), 788-794.

Hosein, R. B., Clarke, A. J., McGuirk, S. P., Griselli, M., Stumper, O., De Giovanni, J. V., Brawn, W. J. (2007). Factors influencing early and late outcome following the Fontan procedure in the current era. The 'Two Commandments'? Eur J Cardiothorac Surg, 31(3), 344-352; discussion 353. doi: $10.1016 /$ j.ejcts.2006.11.043

Husain, S. A., Maldonado, E., Rasch, D., Michalek, J., Taylor, R., Curzon, C., Calhoon, J. H. (2012). Total anomalous pulmonary venous connection: factors associated with mortality and recurrent pulmonary venous obstruction. Ann Thorac Surg, 94(3), 825-831; discussion 831-822. doi: 10.1016/j.athoracsur.2012.04.026

Hyde, J. A., Stumper, O., Barth, M. J., Wright, J. G., Silove, E. D., de Giovanni, J. V., Sethia, B. (1999). Total anomalous pulmonary venous connection: outcome of surgical correction and management of recurrent venous obstruction. Eur J Cardiothorac Surg, 15(6), 735-740; discussion 740-731. 
Jang, S. I., Song, J. Y., Kim, S. J., Choi, E. Y., Shim, W. S., Lee, C., Lee, C. H. (2010). The recent surgical result of total anomalous pulmonary venous return. Korean Circ J, 40(1), 31-35. doi: 10.4070/kcj.2010.40.1.31

Javid, H., Tufo, H. M., Najafi, H., Dye, W. S., Hunter, J. A., \& Julian, O. C. (1969). Neurological abnormalities following open-heart surgery. J Thorac Cardiovasc Surg, 58(4), 502-509.

Jenkins, K. J., Sanders, S. P., Orav, E. J., Coleman, E. A., Mayer, J. E., Jr., \& Colan, S. D. (1993). Individual pulmonary vein size and survival in infants with totally anomalous pulmonary venous connection. J Am Coll Cardiol, 22(1), 201-206.

Johnston, W. E., Vinten-Johansen, J., DeWitt, D. S., O'Steen, W. K., Stump, D. A., \& Prough, D. S. (1991). Cerebral perfusion during canine hypothermic cardiopulmonary bypass: effect of arterial carbon dioxide tension.Ann Thorac Surg, 52(3), 479-489.

Jonas, R. A., Smolinsky, A., Mayer, J. E., \& Castaneda, A. R. (1987). Obstructed pulmonary venous drainage with total anomalous pulmonary venous connection to the coronary sinus. Am J Cardiol, 59(5), 431-435.

Karamlou, T., Gurofsky, R., Al Sukhni, E., Coles, J. G., Williams, W. G., Caldarone, C. A., McCrindle, B. W. (2007). Factors associated with mortality and reoperation in 377 children with total anomalous pulmonary venous connection. Circulation, 115(12), 1591-1598. doi: 10.1161/CIRCULATIONAHA.106.635441

Katz, N. M., Kirklin, J. W., \& Pacifico, A. D. (1978). Concepts and practices in surgery for total anomalous pulmonary venous connection. Ann Thorac Surg, 25(5), 479-487.

Kaulitz, R., Luhmer, I., Bergmann, F., Rodeck, B., \& Hausdorf, G. (1997). Sequelae after modified Fontan operation: postoperative haemodynamic data and organ function. Heart, 78(2), 154159.

Keith, J. D., Rowe, R. D., Vlad, P., \& O'Hanley, J. H. (1954). Complete anomalous pulmonary venous drainage. Am J Med, 16(1), 23-38.

Kirklin, J. W., Dawson, B., Devloo, R. A., \& Theye, R. A. (1961). Open intracardiac operations: use of circulatory arrest during hypothermia induced by blood cooling. Ann Surg, 154, 769-776.

Kirshbom, P. M., Myung, R. J., Gaynor, J. W., Ittenbach, R. F., Paridon, S. M., DeCampli, W. M., Spray, T. L. (2002). Preoperative pulmonary venous obstruction affects long-term outcome for survivors of total anomalous pulmonary venous connection repair. Ann Thorac Surg, 74(5), 1616-1620.

Lacour-Gayet, F. (2006). Surgery for pulmonary venous obstruction after repair of total anomalous pulmonary venous return. Semin Thorac Cardiovasc Surg Pediatr Card Surg Annu, 45-50. doi: 10.1053/j.pcsu.2006.02.010

Lacour-Gayet, F., Rey, C., \& Planche, C. (1996). Pulmonary vein stenosis. Description of a sutureless surgical procedure using the pericardium in situ. Arch Mal Coeur Vaiss, 89(5), 633-636.

Lacour-Gayet, F., Zoghbi, J., Serraf, A. E., Belli, E., Piot, D., Rey, C., Planche, C. (1999). Surgical management of progressive pulmonary venous obstruction after repair of total anomalous pulmonary venous connection. J Thorac Cardiovasc Surg, 117(4), 679-687.

Lamb, R. K., Qureshi, S. A., Wilkinson, J. L., Arnold, R., West, C. R., \& Hamilton, D. I. (1988). Total anomalous pulmonary venous drainage. Seventeen-year surgical experience. J Thorac Cardiovasc Surg, 96(3), 368-375.

Lee, W. H., Jr., Brady, M. P., Rowe, J. M., \& Miller, W. C., Jr. (1971). Effects of extracorporeal circulation upon behavior, personality, and brain function. II. Hemodynamic, metabolic, and psychometric correlations. Ann Surg, 173(6), 1013-1023. 
Lincoln, C. R., Rigby, M. L., Mercanti, C., Al-Fagih, M., Joseph, M. C., Miller, G. A., \& Shinebourne, E. A. (1988). Surgical risk factors in total anomalous pulmonary venous connection. Am J Cardiol, 61(8), 608-611.

Linde, L. M., Rasof, B., \& Dunn, O. J. (1967). Mental development in congenital heart disease. J Pediatr, 71(2), 198-203.

Linderkamp, O., Klose, H. J., Betke, K., Brodherr-Heberlein, S., Buhlmeyer, K., Kelson, S., \& Sengespeik, C. (1979). Increased blood viscosity in patients with cyanotic congenital heart disease and iron deficiency. J Pediatr, 95(4), 567-569.

Lock, J. E., Bass, J. L., Castaneda-Zuniga, W., Fuhrman, B. P., Rashkind, W. J., \& Lucas, R. V., Jr. (1984). Dilation angioplasty of congenital or operative narrowings of venous channels. Circulation, 70(3), 457-464.

Lodge, A. J., Rychik, J., Nicolson, S. C., Ittenbach, R. F., Spray, T. L., \& Gaynor, J. W. (2004). Improving outcomes in functional single ventricle and total anomalous pulmonary venous connection. Ann Thorac Surg, 78(5), 1688-1695. doi: 10.1016/j.athoracsur.2004.04.057

Lupinetti, F. M., Kulik, T. J., Beekman, R. H., 3rd, Crowley, D. C., \& Bove, E. L. (1993). Correction of total anomalous pulmonary venous connection in infancy. J Thorac Cardiovasc Surg, 106(5), 880-885.

Macartney, F. J., Taylor, J. F., Graham, G. R., De Leval, M., \& Stark, J. (1980). The fate of survivors of cardiac surgery in infancy. Circulation, 62(1), 80-91.

Maeda, K., Yamaki, S., Yokota, M., Murakami, A., \& Takamoto, S. (2004). Hypoplasia of the small pulmonary arteries in total anomalous pulmonary venous connection with obstructed pulmonary venous drainage. J Thorac Cardiovasc Surg, 127(2), 448-456. doi: 10.1016/S0022

Mathew, R., Thilenius, O. G., Replogle, R. L., \& Arcilla, R. A. (1977). Cardiac function in total anomalous pulmonary venous return before and after surgery. Circulation, 55(2), 361-370.

Mazzucco, A., Rizzoli, G., Fracasso, A., Stellin, G., Valfre, C., Pellegrino, P., Gallucci, V. (1983). Experience with operation for total anomalous pulmonary venous connection in infancy. $J$ Thorac Cardiovasc Surg, 85(5), 686-690.

McManus, B. M., Luetzeler, J., \& Roberts, W. C. (1982). Total anomalous pulmonary venous connection: survival for 62 years without surgical intervention. Am Heart J, 103(2), 298-301.

Messmer, B. J., Schallberger, U., Gattiker, R., \& Senning, A. (1976). Psychomotor and intellectual development after deep hypothermia and circulatory arrest in early infancy. $J$ Thorac Cardiovasc Surg, 72(4), 495-502.

Michielon, G., Di Donato, R. M., Pasquini, L., Giannico, S., Brancaccio, G., Mazzera, E., Catena, G. (2002). Total anomalous pulmonary venous connection: long-term appraisal with evolving technical solutions. Eur J Cardiothorac Surg, 22(2), 184-191.

Molina, J. E., Einzig, S., Mastri, A. R., Bianco, R. W., Marks, J. A., Rasmussen, T. M., \& Clack, R. M. (1984). Brain damage in profound hypothermia. Perfusion versus circulatory arrest. J Thorac Cardiovasc Surg, 87(4), 596-604.

Morales, D. L., Braud, B. E., Booth, J. H., Graves, D. E., Heinle, J. S., McKenzie, E. D., \& Fraser, C. D., Jr. (2006). Heterotaxy patients with total anomalous pulmonary venous return: improving surgical results. Ann Thorac Surg, 82(5), 1621-1627; discussion 1627-1628. doi: 10.1016/j.athoracsur.2006.05.053

Mueller, C., Dave, H., \& Pretre, R. (2013). Primary correction of total anomalous pulmonary venous return with a modified sutureless technique. Eur J Cardiothorac Surg, 43(3), 635-640. doi: 10.1093/ejcts/ezs376 
Muller, W. H., Jr. (1951). The surgical treatment of transposition of the pulmonary veins. Ann Surg, 134(4), 683-693.

Mullins, C. E., el-Said, G. M., Neches, W. H., Williams, R. L., Vargo, T. A., Nihill, M. R., \& McNamara, D. G. (1973). Balloon atrial septostomy for total anomalous pulmonary venous return. Br Heart J, 35(7), 752-757.

Mustard, W. T., \& Dolan, F. G. (1957). The surgical treatment of total anomalous pulmonary venous drainage. Ann Surg, 145(3), 379-387.

Najm, H. K., Caldarone, C. A., Smallhorn, J., \& Coles, J. G. (1998). A sutureless technique for the relief of pulmonary vein stenosis with the use of in situ pericardium. J Thorac Cardiovasc Surg, 115(2), 468-470.

Neill, C. A. (1956). Development of the pulmonary veins; with reference to the embryology of anomalies of pulmonary venous return. Pediatrics, 18(6), 880-887.

Newburger, J. W., Jonas, R. A., Wernovsky, G., Wypij, D., Hickey, P. R., Kuban, K. C., (1993). A comparison of the perioperative neurologic effects of hypothermic circulatory arrest versus low-flow cardiopulmonary bypass in infant heart surgery. N Engl J Med, 329(15), 1057-1064. doi: 10.1056/NEJM199310073291501

Newfeld, E. A., Wilson, A., Paul, M. H., \& Reisch, J. S. (1980). Pulmonary vascular disease in total anomalous pulmonary venous drainage. Circulation, 61(1), 103-109.

Oelert, H., Schafers, H. J., Stegmann, T., Kallfelz, H. C., \& Borst, H. G. (1986). Complete correction of total anomalous pulmonary venous drainage: experience with 53 patients. Ann Thorac Surg, 41(4), 392-394.

Ovroutski, S., Ewert, P., Alexi-Meskishvili, V., Holscher, K., Miera, O., Peters, B., Berger, F. (2009). Absence of pulmonary artery growth after fontan operation and its possible impact on late outcome. Ann Thorac Surg, 87(3), 826-831. doi: 10.1016/j.athoracsur.2008.10.075

Patel, C. R., Lane, J. R., Spector, M. L., Smith, P. C., \& Crane, S. S. (2005). Totally anomalous pulmonary venous connection and complex congenital heart disease: prenatal echocardiographic diagnosis and prognosis. J Ultrasound Med, 24(9), 1191-1198.

Pizarro, C., Mroczek, T., Gidding, S. S., Murphy, J. D., \& Norwood, W. I. (2006). Fontan completion in infants. [Evaluation Studies]. Ann Thorac Surg, 81(6), 2243-2248; discussion 2248-2249. doi: 10.1016/j.athoracsur.2006.01.016

Raisher, B. D., Grant, J. W., Martin, T. C., Strauss, A. W., \& Spray, T. L. (1992). Complete repair of total anomalous pulmonary venous connection in infancy. J Thorac Cardiovasc Surg, 104(2), 443448.

Rappaport, L. A., Wypij, D., Bellinger, D. C., Helmers, S. L., Holmes, G. L., Barnes, P. D., Newburger, J. W. (1998). Relation of seizures after cardiac surgery in early infancy to neurodevelopmental outcome. Boston Circulatory Arrest Study Group. Circulation, 97(8), 773-779.

Rashkind, W. J., \& Miller, W. W. (1966). Creation of an atrial septal defect without thoracotomy. A palliative approach to complete transposition of the great arteries. JAMA, 196(11), 991-992.

Razzouk, A. J., Gundry, S. R., Chinnock, R. E., Larsen, R. L., Ruiz, C., Zuppan, C. W., \& Bailey, L. L. (1995). Orthotopic transplantation for total anomalous pulmonary venous connection associated with complex congenital heart disease. J Heart Lung Transplant, 14(4), 713-717.

Rebeyka, I. M., Coles, J. G., Wilson, G. J., Watanabe, T., Taylor, M. J., Adler, S. F., (1987). The effect of low-flow cardiopulmonary bypass on cerebral function: an experimental and clinical study. Ann Thorac Surg, 43(4), 391-396. 
Ricci, M., Elliott, M., Cohen, G. A., Catalan, G., Stark, J., de Leval, M. R., \& Tsang, V. T. (2003). Management of pulmonary venous obstruction after correction of TAPVC: risk factors for adverse outcome. Eur J Cardiothorac Surg, 24(1), 28-36; discussion 36.

Richard F. Davis, G. P. G. (2008). Cardiopulmonary Bypass: Principles and Practice.

Romero-Cardenas, A., Vargas-Barron, J., Rylaarsdam, M., Stumper, O., Villegas, M., Bandin, M. A., Molina, J. (1991). Total anomalous pulmonary venous return: diagnosis by transesophageal echocardiography. Am Heart J, 121(6 Pt 1), 1831-1834.

Sadiq, M., Stumper, O., De Giovanni, J. V., Wright, J. G., Sethia, B., Brawn, W. J., \& Silove, E. D. (1996). Management and outcome of infants and children with right atrial isomerism. Heart, 75(3), 314-319.

Samanek, M., Benesova, D., Goetzova, J., \& Hrycejova, I. (1988). Distribution of age at death in children with congenital heart disease who died before the age of 15. Br Heart J, 59(5), 581585.

Sano, S., Brawn, W. J., \& Mee, R. B. (1989). Total anomalous pulmonary venous drainage. J Thorac Cardiovasc Surg, 97(6), 886-892.

Saxena, A., Fong, L. V., Lamb, R. K., Monro, J. L., Shore, D. F., \& Keeton, B. R. (1991). Cardiac arrhythmias after surgical correction of total anomalous pulmonary venous connection: late follow-up. Pediatr Cardiol, 12(2), 89-91. doi: 10.1007/BF02238409

Schafers, H. J., Luhmer, I., \& Oelert, H. (1987). Pulmonary venous obstruction following repair of total anomalous pulmonary venous drainage. [Case Reports]. Ann Thorac Surg, 43(4), 432-434.

Seale, A. N., Uemura, H., Webber, S. A., Partridge, J., Roughton, M., Ho, S. Y., British Congenital Cardiac, A. (2010). Total anomalous pulmonary venous connection: morphology and outcome from an international population-based study. Circulation, 122(25), 2718-2726. doi: 10.1161/CIRCULATIONAHA.110.940825

Seale, A. N., Uemura, H., Webber, S. A., Partridge, J., Roughton, M., Ho, S. Y., British Congenital Cardiac, A. (2013). Total anomalous pulmonary venous connection: outcome of postoperative pulmonary venous obstruction. J Thorac Cardiovasc Surg, 145(5), 1255-1262. doi: 10.1016/j.jtcvs.2012.06.031

Serraf, A., Bruniaux, J., Lacour-Gayet, F., Chambran, P., Binet, J. P., Lecronier, G., Planche, C. (1991). Obstructed total anomalous pulmonary venous return. Toward neutralization of a major risk factor. J Thorac Cardiovasc Surg, 101(4), 601-606.

Settergren, G., Ohqvist, G., Lundberg, S., Henze, A., Bjork, V. O., \& Persson, B. (1982). Cerebral blood flow and cerebral metabolism in children following cardiac surgery with deep hypothermia and circulatory arrest. Clinical course and follow-up of psychomotor development. Scand J Thorac Cardiovasc Surg, 16(3), 209-215.

Shimokawa, S., Morishita, Y., Maruko, M., Watanabe, S., Taira, A., \& Nakamura, K. (1986). Total anomalous pulmonary venous connection: successful total correction in a 43-year-old man. Nihon Kyobu Geka Gakkai Zasshi, 34(1), 123-128.

Silove, E. D., Behrendt, D. M., Aberdeen, E., \& Bonham-Carter, R. (1972). Total anomalous pulmonary venous drainage. II. Spontaneous functional closure of interatrial communication after surgical correction in infancy. Circulation, 46(2), 357-367.

Sinzobahamvya, N., Arenz, C., Brecher, A. M., Blaschczok, H. C., \& Urban, A. E. (1996). Early and longterm results for correction of total anomalous pulmonary venous drainage (TAPVD) in neonates and infants. Eur J Cardiothorac Surg, 10(6), 433-438. 
Smallhorn, J. F., Burrows, P., Wilson, G., Coles, J., Gilday, D. L., \& Freedom, R. M. (1987). Twodimensional and pulsed Doppler echocardiography in the postoperative evaluation of total anomalous pulmonary venous connection. Circulation, 76(2), 298-305.

Sotaniemi, K. A. (1983). Cerebral outcome after extracorporeal circulation. Comparison between prospective and retrospective evaluations. [Comparative Study]. Arch Neurol, 40(2), 75-77.

Sotaniemi, K. A., Juolasmaa, A., \& Hokkanen, E. T. (1981). Neuropsychologic outcome after openheart surgery. Arch Neurol, 38(1), 2-8.

Stark, J., Smallhorn, J., Huhta, J., de Leval, M., Macartney, F. J., Rees, P. G., \& Taylor, J. F. (1983). Surgery for congenital heart defects diagnosed with cross-sectional echocardiography. Circulation, 68(3 Pt 2), II129-138.

Swain, J. A., McDonald, T. J., Jr., Griffith, P. K., Balaban, R. S., Clark, R. E., \& Ceckler, T. (1991). Lowflow hypothermic cardiopulmonary bypass protects the brain. $J$ Thorac Cardiovasc Surg, 102(1), 76-83; discussion 83-74.

Tanel, R. E., Kirshbom, P. M., Paridon, S. M., Hartman, D. M., Burnham, N. B., McBride, M. G., Gaynor, J. W. (2007). Long-term noninvasive arrhythmia assessment after total anomalous pulmonary venous connection repair. Am Heart J, 153(2), 267-274. doi: 10.1016/j.ahj.2006.11.003

Terplan, K. L. (1973). Patterns of brain damage in infants and children with congental heart disease. Association with catheterization and surgical procedures. Am J Dis Child, 125(2), 176-185.

Tharion, J., Johnson, D. C., Celermajer, J. M., Hawker, R. M., Cartmill, T. B., \& Overton, J. H. (1982). Profound hypothermia with circulatory arrest: nine years' clinical experience. $J$ Thorac Cardiovasc Surg, 84(1), 66-72.

Treasure, T., Naftel, D. C., Conger, K. A., Garcia, J. H., Kirklin, J. W., \& Blackstone, E. H. (1983). The effect of hypothermic circulatory arrest time on cerebral function, morphology, and biochemistry. An experimental study. J Thorac Cardiovasc Surg, 86(5), 761-770.

Turley, K., Tucker, W. Y., Ullyot, D. J., \& Ebert, P. A. (1980). Total anomalous pulmonary venous connection in infancy: Influence of age and type of lesion. Am J Cardiol, 45(1), 92-97. doi: 10.1016/0002-9149(80)90225-8

Valsangiacomo, E. R., Hornberger, L. K., Barrea, C., Smallhorn, J. F., \& Yoo, S. J. (2003). Partial and total anomalous pulmonary venous connection in the fetus: two-dimensional and Doppler echocardiographic findings. Ultrasound Obstet Gynecol, 22(3), 257-263. doi: 10.1002/uog.214

Wang, J. K., Lue, H. C., Wu, M. H., Young, M. L., Wu, F. F., \& Wu, J. M. (1993). Obstructed total anomalous pulmonary venous connection. Pediatr Cardiol, 14(1), 28-32.

Wang, P. Y., Hwang, B. T., Lu, J. H., Lee, P. C., Tiu, C. M., Weng, Z. C., \& Meng, L. C. (2004). Significance of pulmonary venous obstruction in total anomalous pulmonary venous return. $J$ Chin Med Assoc, 67(7), 331-335.

Weiss, M., Piwnica, A., Lenfant, C., Sprovieri, L., Laurent, D., Blondeau, P., \& Dubost, C. (1960). Deep hypothermia with total circulatory arrest. Trans Am Soc Artif Intern Organs, 6, 227-239.

Wells, F. C., Coghill, S., Caplan, H. L., \& Lincoln, C. (1983). Duration of circulatory arrest does influence the psychological development of children after cardiac operation in early life. $J$ Thorac Cardiovasc Surg, 86(6), 823-831.

Wilson, J. $(1798,1798)$. A descripton of a very unusual formation of the human heart. Phil Trans Royal Society, (1798.346-356.). 
Wilson, W. R., Jr., Ilbawi, M. N., DeLeon, S. Y., Quinones, J. A., Arcilla, R. A., Sulayman, R. F., \& Idriss, F. S. (1992). Technical modifications for improved results in total anomalous pulmonary venous drainage. J Thorac Cardiovasc Surg, 103(5), 861-870; discussion 870-861.

Wright, J. S., Hicks, R. G., \& Newman, D. C. (1979). Deep hypothermic arrest: observations on later development in children. J Thorac Cardiovasc Surg, 77(3), 466-468.

Wukasch, D. C., Deutsch, M., Reul, G. J., Hallman, G. L., \& Cooley, D. A. (1975). Total anomalous pulmonary venous return. Review of 125 patients treated surgically. Ann Thorac Surg, 19(6), 622-633.

Yoshimura, N., Fukahara, K., Yamashita, A., Doki, Y., Doi, T., Takeuchi, K., Nagura, S. (2012). Management of pulmonary venous obstruction. Gen Thorac Cardiovasc Surg, 60(12), 785791. doi: 10.1007/s11748-012-0154-8

Yoshimura, N., Yamaguchi, M., Oshima, Y., Oka, S., Ootaki, Y., Tei, T., \& Kido, S. (2001). Risk factors influencing early and late mortality after total cavopulmonary connection. Eur J Cardiothorac Surg, 20(3), 598-602.

Yun, T. J., Coles, J. G., Konstantinov, I. E., Al-Radi, O. O., Wald, R. M., Guerra, V., Caldarone, C. A. (2005). Conventional and sutureless techniques for management of the pulmonary veins: Evolution of indications from postrepair pulmonary vein stenosis to primary pulmonary vein anomalies. J Thorac Cardiovasc Surg, 129(1), 167-174. doi: 10.1016/j.jtcvs.2004.08.043 


\section{Anhang}

\section{Danksagung}

Ich danke Herrn Prof. Dr. Lange, der mit das Thema zu dieser Dissertation überlassen hat.

Herrn PD Dr. Hörer gilt ganz besonderer Dank. Sein Enthusiasmus, seine Geduld und seine heitere, motivierende Art scheinen auch über die Grenzen hinaus nicht trübbar und haben mir zu jedem Zeitpunkt bis zur Vollendung der Arbeit Kraft gegeben.

Last but not least danke ich meiner Mama und meinem Papi, die mir beide in ihrer individuellen Art und Weise mit Rat und Tat über all die Jahre zur Seite standen und stets wussten, die durch alltägliche Umstände erschwerten Durststrecken zu verkürzen. 